\title{
A New Calculation of the lonizing Background Spectrum and the Effects of He li Reionization
}

\section{Citation}

Faucher-Giguère, Claude-André, Adam Lidz, Matias Zaldarriaga, and Lars Hernquist. 2009. "A NEW CALCULATION OF THE IONIZING BACKGROUND SPECTRUM AND THE EFFECTS OF He II REIONIZATION." The Astrophysical Journal 703 (2): 1416-43. https:// doi.org/10.1088/0004-637x/703/2/1416.

\section{Permanent link}

http://nrs.harvard.edu/urn-3:HUL.InstRepos:41381821

\section{Terms of Use}

This article was downloaded from Harvard University's DASH repository, and is made available under the terms and conditions applicable to Other Posted Material, as set forth at http:// nrs.harvard.edu/urn-3:HUL.InstRepos:dash.current.terms-of-use\#LAA

\section{Share Your Story}

The Harvard community has made this article openly available. Please share how this access benefits you. Submit a story.

Accessibility 


\title{
A NEW CALCULATION OF THE IONIZING BACKGROUND SPECTRUM AND THE EFFECTS OF He II REIONIZATION
}

\author{
Claudde-André Faucher-Giguère ${ }^{1}$, Adam Lidz ${ }^{1}$, Matias Zaldarriaga ${ }^{1,2}$, and Lars Hernquist \\ ${ }^{1}$ Department of Astronomy, Harvard University, Cambridge, MA 02138, USA; cgiguere@ cfa.harvard.edu \\ 2 Jefferson Physical Laboratory, Harvard University, Cambridge, MA 02138, USA \\ Received 2009 January 28; accepted 2009 August 13; published 2009 September 9
}

\begin{abstract}
The ionizing background determines the ionization balance and the thermodynamics of the cosmic gas. It is therefore a fundamental ingredient to theoretical and empirical studies of both the intergalactic medium (IGM) and galaxy formation. We present here a new calculation of its spectrum that satisfies the empirical constraints we recently obtained by combining state-of-the-art luminosity functions and intergalactic opacity measurements. In our preferred model, star-forming galaxies and quasars each contribute substantially to the $\mathrm{H}$ I ionizing field at $z<3$, with galaxies rapidly overtaking quasars at higher redshifts as quasars become rarer. In addition to our fiducial model, we explore the physical dependences of the calculated background and clarify how recombination emission contributes to the ionization rates. We find that recombinations do not simply boost the ionization rates by the number of re-emitted ionizing photons as many of these rapidly redshift below the ionization edges and have a distribution of energies. A simple analytic model that captures the main effects seen in our numerical radiative transfer calculations is given. Finally, we discuss the effects of He II reionization by quasars on both the spectrum of the ionizing background and on the thermal history of the IGM. In regions that have yet to be reionized, the spectrum is expected to be almost completely suppressed immediately above $54.4 \mathrm{eV}$, while a background of higher energy $(\gtrsim 0.5 \mathrm{keV})$ photons permeates the entire universe owing to the frequency dependence of the photoionization cross section. We provide an analytical model of the heat input during He II reionization and its effects on the temperature-density relation.
\end{abstract}

Key words: cosmology: theory - diffuse radiation - galaxies: evolution - galaxies: formation - galaxies: high-redshift - quasars: absorption lines

Online-only material: color figure

\section{INTRODUCTION}

The cosmic baryons give the ultraviolet (UV) background a particularly important standing among radiation backgrounds. In fact, the ionization potentials of both hydrogen and helium, ${ }^{3}$ which together account for $99 \%$ of the baryonic mass density (e.g., Burles et al. 2001), correspond to electromagnetic wavelengths in the UV regime. The UV background therefore governs the ionization state of intergalactic gas and furthermore plays a key role in its thermal evolution through photoheating. As such, it is an essential input to cosmological hydrodynamic simulations (e.g., Hernquist et al. 1996; Katz et al. 1996b; Davé et al. 1999; Springel \& Hernquist 2003) as well as to observational studies of the intergalactic medium (IGM).

The ionizing background can for example suppress the abundance of dwarf galaxies and the amount of cool gas in low-mass galaxies that do form both by modifying the cooling function through the ionization balance and by heating the gas before it collapses (Efstathiou 1992; Quinn et al. 1996; Thoul \& Weinberg 1996; Weinberg et al. 1997). It is also crucially important for any simulation of the Ly $\alpha$ forest, since the absence of a Gunn \& Peterson (1965) trough in the spectra of quasars up to $z \sim 6$ (e.g., Fan et al. 2002, 2006b; Becker et al. 2007) indicates that the IGM is highly ionized up to at least that redshift. Since the optical depth of the Ly $\alpha$ forest is directly tied to the hydrogen photoionization rate (e.g., Rauch et al. 1997; McDonald \& Miralda-Escudé 2001; Meiksin \& White 2003; Tytler et al. 2004; Kirkman et al. 2005; Bolton et al. 2005; Faucher-Giguère et al. 2008a, 2008b), it is important

\footnotetext{
13.6, 24.6, and $54.6 \mathrm{eV}$ for $\mathrm{H} \mathrm{I}, \mathrm{He} \mathrm{I}$, and $\mathrm{He}$ II, respectively.
}

to know the latter accurately. In addition, the UV background determines the photoionization rate of helium, which is of particular relevance given the growing interest in studying He II reionization, which may occur at redshifts $z \sim 3-4$ for which a wealth of observational data is already available and upcoming (Section 7). The full spectrum of the UV background is perhaps most important in the study of metal ions, such as S IV and C IV, where relating the ionic abundances to elemental abundances or cosmic metal mass density requires ionizing corrections (e.g., Cowie et al. 1995; Songaila \& Cowie 1996; Songaila 2001; Schaye et al. 2003; Boksenberg et al. 2003; Aguirre et al. 2004; Simcoe et al. 2004; Aguirre et al. 2008; Fechner \& Richter 2009). Finally, the spectrum of the UV background obviously depends on its sources, and its study can therefore teach much about the sources responsible for keeping the IGM ionized, as well as reionizing it (e.g., Miralda-Escudé 2003; Bolton \& Haehnelt 2007; Faucher-Giguère et al. 2008a, 2008b).

Following early work (Miralda-Escude \& Ostriker 1990; Shapiro et al. 1994; Giroux \& Shapiro 1996), Haardt \& Madau (1996; see also Fardal et al. 1998) pioneered calculations of the UV background spectrum in their study of radiative transfer in a clumpy universe. Their model and some variants (e.g., Haardt \& Madau 2001) have since been extensively used in several hundreds of studies in the literature. Over a decade after their original calculation, the empirical constraints on the UV backgrounds and its sources have however improved dramatically. Larger and deeper surveys at all wavelengths have constrained the quasar luminosity function to both fainter magnitudes and higher redshifts (e.g., Boyle et al. 2000; Miyaji et al. 2000; Fan et al. 2001; Ueda et al. 2003; Fan et al. 2004; Croom et al. 2004; Richards et al. 2005; Barger et al. 2005; 
Hasinger et al. 2005; Richards et al. 2006; Brown et al. 2006; Hopkins et al. 2007; Fontanot et al. 2007; Siana et al. 2008). At the same time, our understanding of the population of high-redshift star-forming galaxies has tremendously expanded thanks to the application of the Lyman break selection technique to ever more ambitious surveys (e.g., Steidel et al. 1999; Sawicki \& Thompson 2006; Yoshida et al. 2006; Bouwens et al. 2007; Reddy et al. 2008). Detailed studies of the absorption properties of the IGM, particularly by H I and He II, have also provided particularly valuable constraints on the UV background. These constraints are especially relevant for the UV background as the IGM is sensitive to the integral of the UV photons emitted by all sources, regardless of whether these are directly detected. Moreover, the IGM constraints probe the density of ionizing photons and thus circumvent the need to assume an escape fraction relating the luminosity of quasars and galaxies measured redward of the Lyman limit to their net output of ionizing radiation.

In a series of previous papers, we have measured the intergalactic Ly $\alpha$ opacity (Faucher-Giguère et al. 2008d) and derived empirical constraints on the UV background and its sources incorporating also information on the reionization of $\mathrm{H} \mathrm{I}$ and He II, as well as $N_{\mathrm{He} \text { II }} / N_{\mathrm{HI}}$ column density ratios (FaucherGiguère et al. 2008a, 2008b). Specifically, we found that the H I photoionization rate is remarkably constant over the redshift interval $z=2-4$. Since the quasar luminosity function peaks strongly around $z=2$, star-forming galaxies most likely dominate the ionizing background beyond $z \approx 3$. The column density ratios however indicate that quasars likely do contribute a large fraction of the ionizing background at their peak. In this paper, we use these constraints as a basis for a new calculation of the full spectrum of the UV background. In addition to the improved empirical input, we re-examine many of the assumptions entering the original Haardt \& Madau (1996) calculation. As we will show, we find that the original calculation likely overestimated the contribution of recombination emission to the ionizing background by a factor of a few. Of perhaps greatest interest, the original calculation completely neglected the effects of He II reionization, so that simulators have usually resorted to artificial prescriptions to complement the Haardt \& Madau (1996) spectrum. Here, we explicitly discuss the effects of He II reionization on the UV background spectrum as well as on the thermal history of the IGM and provide a physical framework to implement them.

We review the basic equations of cosmological radiative transfer and the column density distribution of $\mathrm{HI}$ absorbers in Section 2. In Section 3, we study the ionization structure of individual absorbers and derive approximations to be used in the cosmological solution. Section 4 is devoted to the calculation of the contribution recombinations to the cosmological emissivity. Empirically calibrated calculations of the UV background spectrum, derived quantities, and their dependences on input parameters are presented in Section 5. In Section 6, we investigate how the calculated spectra (including recombination emission) and the corresponding ionization rates depend on input parameters. The effects of $\mathrm{He}$ II reionization are investigated in Section 7. We finally compare our results with previous work in Section 8 and conclude in Section 9.

A series of appendices supplement the main text with technical details. In Appendix A, we describe our photoionization code. Appendix B contains technical aspects of our treatment of recombination emission, while Appendix $C$ presents an analytic model of how this recombination emission boosts the
Table 1

Symbols used in this Work

\begin{tabular}{lc}
\hline \hline Symbol & Definition \\
\hline$n_{i}$ & Number density of species $i$ \\
$N_{i}$ & Column density of species $i$ \\
$\tau_{v}$ & Optical depth at frequency $v$ \\
$\bar{\tau}$ & Effective optical depth \\
$I_{v}$ & Specific intensity along a ray \\
$J_{v}$ & Angle-averaged specific intensity \\
$\alpha_{v}{ }^{a}$ & Absorption coefficient \\
$j_{v}$ & Emission coefficient \\
$T$ & Gas temperature \\
$\sigma_{i}$ & Photoionization cross section of species $i$ \\
$\Gamma_{i}$ & Photoionization rate of species $i$ \\
$\alpha_{i}^{A, B}$ & Case A or B recombination coefficient to species $i$ \\
$\alpha_{i, n=j}$ & Recombination coefficient directly to level $n=j$ of species $i$ \\
$\phi(v)$ & Line profile \\
$X$ & Mass fraction of hydrogen \\
$Y$ & Mass fraction of helium \\
$x_{\mathrm{II}}$ & Fraction of hydrogen in H II \\
$y_{\mathrm{II}}, y_{\mathrm{III}}$ & Fractions of helium in He II, He III \\
\hline
\end{tabular}

Note. ${ }^{\text {a }}$ The symbol $\alpha$ is also sometimes used as a recombination coefficient or a spectral index. The meaning should be transparent from the context.

photoionization rates. Appendix D analytically discusses spectral filtering in different regimes to aid in interpreting our results. Appendix E finally references atomic physics quantities used in our calculations.

Throughout, we assume a cosmology with $\left(\Omega_{\mathrm{m}}, \Omega_{\mathrm{b}}, \Omega_{\Lambda}\right.$, $\left.h, \sigma_{8}\right)=(0.28,0.046,0.72,0.70,0.82)$, as inferred from the Wilkinson Microwave Anisotropy Probe (WMAP) five-year data in combination with baryon acoustic oscillations and supernovae (Komatsu et al. 2009). Unless otherwise stated, all error bars are $1 \sigma$. Table 1 defines many symbols used here.

\section{COSMOLOGICAL RADIATIVE TRANSFER}

\subsection{Radiative Transfer Equations}

In this work, we are first concerned with the specific intensity of the diffuse cosmological UV background averaged over both space and angle, which we denote by $J_{v}$. The basic equations of cosmological radiative transfer were particularly well summarized by Haardt \& Madau (1996), on which we base our treatment below. The specific intensity satisfies the radiative transfer equation

$$
\left(\frac{\partial}{\partial t}-v H \frac{\partial}{\partial v}\right) J_{v}=-3 H J_{v}-c \alpha_{v} J_{v}+\frac{c}{4 \pi} \epsilon_{v},
$$

where $H(t)$ is the Hubble parameter, $c$ is the speed of light, $\alpha_{v}$ is the proper absorption coefficient, and $\epsilon_{v}$ is the proper emissivity. Integrating Equation (1) and expressing the result in terms of redshift gives

$$
J_{v_{0}}\left(z_{0}\right)=\frac{1}{4 \pi} \int_{z_{0}}^{\infty} d z \frac{d l}{d z} \frac{\left(1+z_{0}\right)^{3}}{(1+z)^{3}} \epsilon_{v}(z) \exp \left[-\bar{\tau}\left(v_{0}, z_{0}, z\right)\right]
$$

where $v=v_{0}(1+z) /\left(1+z_{0}\right)$, the proper line element $d l / d z=$ $c /[(1+z) H(z)]$, and the "effective optical depth" $\bar{\tau}^{4}$ quantifies

\footnotetext{
4 This quantity is often denoted by $\tau_{\text {eff }}$. We use a different notation here to distinguish it from the effective optical depth $\tau_{\text {eff }}(z)=-\ln \langle F\rangle(z)$ owing to Ly $\alpha$ absorption measured from quasar spectra (e.g., Faucher-Giguère et al. 2008d).
} 
the attenuation of photons of frequency $v_{0}$ at redshift $z_{0}$ that were emitted at redshift $z$ by the relation $e^{\bar{\tau}}=\left\langle e^{-\tau}\right\rangle$, where the average is over all lines of sights from $z_{0}$ to $z$. For Poissondistributed absorbers, each of column density $N_{\mathrm{H} \text { I }}$

$$
\bar{\tau}\left(v_{0}, z_{0}, z\right)=\int_{z_{0}}^{z} d z^{\prime} \int_{0}^{\infty} d N_{\mathrm{H}_{\mathrm{I}}} \frac{\partial^{2} N}{\partial N_{\mathrm{H}_{\mathrm{I}}} \partial z^{\prime}}\left(1-e^{-\tau_{v}}\right),
$$

where $\partial^{2} N / \partial N_{\mathrm{H}_{\mathrm{I}}} \partial z^{\prime}$ is the column density distribution versus redshift (Paresce et al. 1980).

Note that these expressions neglect the clustering of sources and sinks of radiation in both the Poisson distribution assumption and in assuming that the spatial average $\left\langle\epsilon_{v}(z) \exp \left[-\tau\left(v_{0}, z_{0}, z\right)\right]\right\rangle$ separates into $\left\langle\epsilon_{v}(z)\right\rangle\left\langle\exp \left[-\tau\left(v_{0}, z_{0}\right.\right.\right.$, $z)]\rangle$ in the integrand of Equation (2). The Poisson distribution assumption should be very good since the mean free path of ionizing photons of hundreds of comoving $\mathrm{Mpc}$ at most redshifts of interest (see Section 7) far exceeds the correlation length $\lesssim 5$ comoving Mpc of the Ly $\alpha$ forest absorbers (e.g., McDonald et al. 2000; Faucher-Giguère et al. 2008c). While absorbers likely do cluster around sources, this effect can be viewed as being incorporated into the definition of the escape fraction. The above formalism will however break down in certain regimes where sources are rare and in particular during He II reionization. We discuss these cases in Section 7. As in Equation (2), we henceforth drop the explicit averaging brackets around the emissivity $\epsilon_{v}$.

The optical depth $\tau_{v}$ shortward of the Lyman limit will be dominated by the photoelectric opacity of hydrogen and helium

$$
\tau_{v}=N_{\mathrm{HI}_{\mathrm{I}}} \sigma_{\mathrm{HI}}(v)+N_{\mathrm{He}_{\mathrm{I}}} \sigma_{\mathrm{HeI}}(v)+N_{\mathrm{He}} \sigma_{\mathrm{He}}(v),
$$

where $N_{i}$ and $\sigma_{i}$ are the column densities and photoionization cross sections of ion $i$. Only the distribution of $N_{\mathrm{H}}$ is reasonably well determined over a large redshift interval. We will therefore make use of relations between $N_{\mathrm{H}_{\mathrm{I}}}$ and the column densities of helium established in Section 3. In our calculations, we will prescribe $\partial^{2} N / \partial N_{\mathrm{HI}} \partial z$ as well as the specific emissivity of the ionizing sources, $\epsilon_{v}^{\mathrm{src}}$, based on our previous empirical studies (Faucher-Giguère et al. 2008d, 2008a, 2008b).

\subsection{H I Column Density Distribution}

Following previous work and consistent with empirical constraints, we parameterize the column density distribution with power laws in $N_{\mathrm{HI}}$ and $z$ :

$$
\frac{\partial^{2} N}{\partial z \partial N_{\mathrm{HI}}}= \begin{cases}N_{0, \text { low }} N_{\mathrm{HI}}^{-\beta}(1+z)^{\gamma_{\text {low }}} & z \leqslant z_{\text {low }} \\ N_{0} N_{\mathrm{HI}}^{-\beta}(1+z)^{\gamma} & z>z_{\text {low }} .\end{cases}
$$

The transition at $z_{\text {low }}$ accounts for the flattening of the redshift evolution observed at $z \lesssim 1.5$ (e.g., Weymann et al. 1998; Kim et al. 2001, 2002) theoretically understood to arise from the drop in intensity of the ionizing background at low redshifts (Theuns et al. 1998; Davé et al. 1999; Bianchi et al. 2001; Scott et al. 2002). We fix $\gamma_{\text {low }}=0.2$.

For a steep column density distribution with $\beta<2$, most of the contribution to $\bar{\tau}$ at the Lyman limit arises from systems of optical depth near unity $\left(N_{\mathrm{H}_{\mathrm{I}}} \approx 10^{17.2} \mathrm{~cm}^{-2}\right)$. We thus focus on the values of the power-law indices $\beta$ and $\gamma$ that are most appropriate in this neighborhood. Stengler-Larrea et al. (1995) find that $d N / d z=C(1+z)^{\gamma}$ with $C=0.25$, and $\gamma=1.5$ provides a good fit at least up to $z=4.1$ for systems with $N_{\mathrm{HI}} \geqslant 10^{17.2} \mathrm{~cm}^{-2}$, whereas the column density power law is well fitted by $\beta=1.4$ (Misawa et al. 2007). The constant $N_{0}$ in Equation (5) is related to $C$ by $N_{0}=(\beta-1) C N_{\mathrm{H} \text {, } m i n}^{\beta-1}$, where $N_{\mathrm{H} \text {, min }}=\sigma_{\mathrm{HI}}^{-1}=10^{17.2} \mathrm{~cm}^{-2}$. We use these values, with $z_{\text {low }}=1.5$, in fiducial calculations but explore varying these parameters in Section 6.

Before proceeding, we note that column density distribution is largely unconstrained above $z=4.1$, the highest redshift at which the Lyman limit system abundance has been measured. We therefore simply extrapolate from lower redshifts and caution that our calculation of the ionizing background may become inaccurate in this regime. In particular, we expect the extrapolation to become unreliable at $z \approx 5.7$, where the evolution of the effective optical depth measured from the spectra of $z \geqslant 6$ quasars diverges rapidly from the power law fitting the data below this redshift (Fan et al. 2006b; though see Becker et al. 2007 for an opposing point of view), perhaps owing to $\mathrm{H}$ I reionization.

\section{THE IONIZATION STRUCTURE OF INDIVIDUAL ABSORBERS}

\subsection{Overview}

In this section, we study the photoionization equilibrium structure of individual cosmic absorbers composed of hydrogen and helium (with mass fraction $75 \%$ and $25 \%$, respectively) as a function of the illuminating radiation background. This serves two purposes: to close the set of equations of cosmological radiative transfer (Section 2) and to allow us to more realistically calculate the contribution of recombination lines to the ionizing background spectrum (Section 4). To this end, we have developed a code that self-consistently solves the photoionization equilibrium balance, including the influence of recombination radiation. This code provides more accurate solutions than previous approximations with semi-infinite geometry and an escape probability formalism (Haardt \& Madau 1996) or gray cross sections (Fardal et al. 1998). To alleviate the text, the details of our photoionization calculations are provided in Appendix A. We assume our absorbers to be slabs of thickness equal to the Jeans scale of the gas, which is a function of the assumed temperature $T=2 \times 10^{4} \mathrm{~K}$ Schaye (2001). This temperature is consistent with the line-fitting analysis of McDonald et al. (2001) and with the Ly $\alpha$ forest power spectrum analysis of Zaldarriaga et al. (2001) for the $z \sim 2-4$ IGM at mean density.

$$
\text { 3.2. } N_{\mathrm{He} \text { II }} \text { and } N_{\mathrm{He} \text { I }} \text { from } N_{\mathrm{H} \text { I }}
$$

As only the column density distribution of $\mathrm{H}$ i is reasonably well constrained, the first application of our photoionization calculations is to obtain relations giving $N_{\mathrm{He} \text { II }}$ and $N_{\mathrm{He}}$ in terms of $N_{\mathrm{HI}_{\mathrm{I}}}$. In Figure 1, we show the numerical results for external spectra $J_{v}=10^{-21} \mathrm{erg} \mathrm{s}^{-1} \mathrm{~cm}^{-2} \mathrm{~Hz}^{-1} \mathrm{sr}^{-1}\left(v / \nu_{\mathrm{HI}}\right)^{-\alpha}$ with $\alpha=0.0,0.5,1.0,1.5,2.0$, and 2.5 from bottom up. To ensure that these test spectra are representative of the UV background, we suppress the power laws by a factor of 10 above the He II ionization edge.

Since these relations enter within three nested integrals (Equations (1) and (2)), it is necessary to develop analytical approximations that are fast to evaluate. It would be impractical to use self-consistent numerical photoionization calculations at each redshift and for each column density in the cosmological solution. Defining $\eta \equiv N_{\mathrm{He} \text { II }} / N_{\mathrm{HI}}$, when both $\mathrm{H}_{\text {I }}$ and He II are optically thin, and in the limit of nearly complete ionization we 

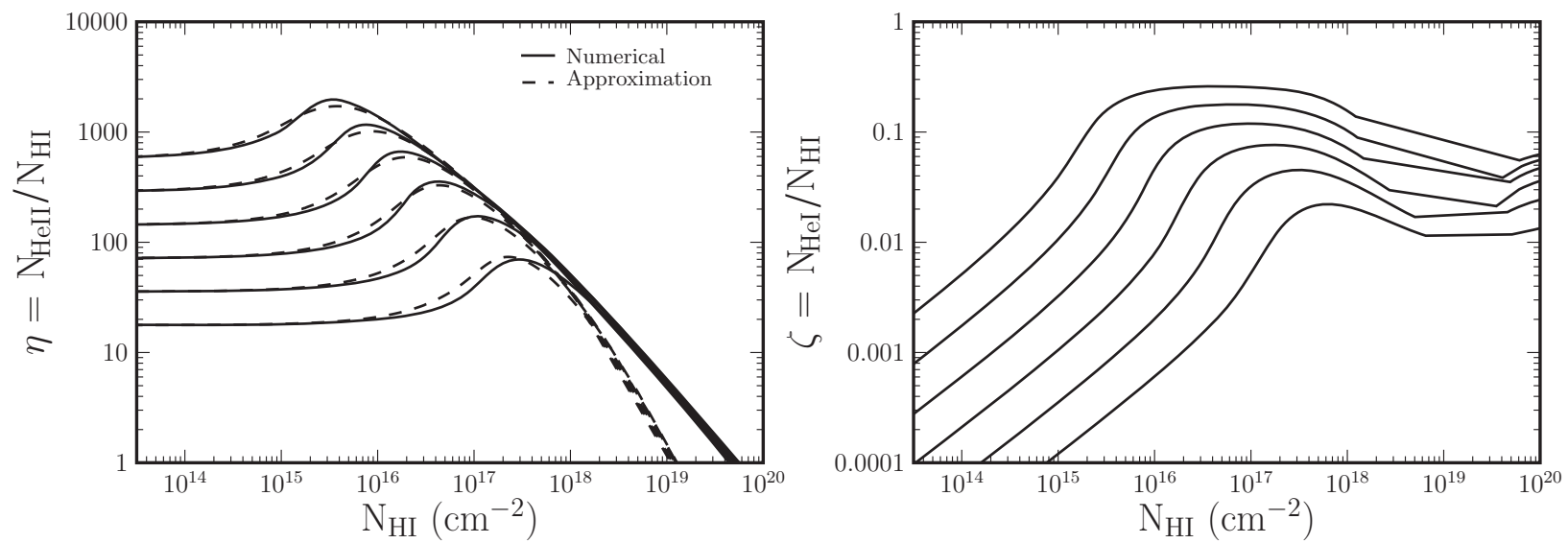

Figure 1. Ratio $\eta=N_{\mathrm{He} \text { II }} / N_{\mathrm{HI}}$ (left) and $\zeta=N_{\mathrm{HeI}} / N_{\mathrm{HI}}$ (right) as a function of H I column density. The solid curves show full numerical photoionization calculations and the dashed ones show analytical approximations based on Equation (7) for $\eta$. From the bottom up, power-law external spectra $J_{v}=$ $10^{-21} \mathrm{erg} \mathrm{s}^{-1} \mathrm{~cm}^{-2} \mathrm{~Hz}^{-1} \mathrm{sr}^{-1}\left(v / \nu_{\mathrm{HI}}\right)^{-\alpha}$ with $\alpha=0.0,0.5,1.0,1.5,2.0$, and 2.5 , suppressed by a factor of 10 above the He II ionization edge, are assumed.

have

$$
\eta_{\text {thin }}=\frac{\Gamma_{\mathrm{HI}_{\mathrm{I}}}}{\Gamma_{\mathrm{He} \text { II }}} \frac{\alpha_{\mathrm{He} \text { II }}^{\mathrm{A}}}{\alpha_{\mathrm{HI}}^{\mathrm{A}}} \frac{Y}{4 X} .
$$

For fixed external background and increasing $N_{\mathrm{H}}$, an absorber first becomes optically thick in He II, at which point $\eta$ increases rapidly with $N_{\mathrm{H}_{\mathrm{I}}}$. The absorber then becomes optically thick in $\mathrm{H}_{\mathrm{I}}$ as well and, owing to the greater abundance of hydrogen, $N_{\mathrm{H} \text { I }}$ finally rapidly overtakes $N_{\mathrm{He} \text { II }}$. This leads to the plateau, increase, and then decrease of $\eta$ with respect to $N_{\mathrm{HI}}$ seen in the numerical calculations. Similar behavior is found in threedimensional radiative transfer simulations of the IGM (Maselli \& Ferrara 2005).

Fardal et al. (1998) give a fitting formula derived under the assumptions of negligible $N_{\mathrm{He}}$ and $n_{\mathrm{HI}} / n_{\mathrm{H}} \ll 1$ :

$$
\frac{Y}{16 X} \frac{\tau_{\mathrm{HI}}}{1+A \tau_{\mathrm{HI}}} I_{\mathrm{HI}}=\tau_{\mathrm{He} \text { II }}+\frac{\tau_{\mathrm{He} \text { II }}}{1+B \tau_{\mathrm{He} \text { II }}} I_{\mathrm{He} \mathrm{II}},
$$

where $I_{\mathrm{i}} \equiv \Gamma_{\mathrm{i}}^{\text {ext }} / n_{e} \alpha_{\mathrm{i}}^{\mathrm{A}}, \tau_{\mathrm{HI}}=\sigma_{\mathrm{HI}_{\mathrm{I}}} N_{\mathrm{HI}}$ and $\tau_{\mathrm{He} \text { II }}=\sigma_{\mathrm{He}} N_{\mathrm{He}}{ }_{\mathrm{HI}}$, and we have generalized their result to allow for arbitrary coefficients $A$ and $B$. These fitting coefficients depend on, in particular, the relation between $N_{\mathrm{HI}}$ and $n_{e}$, and we will not be using the same model as these authors. Although our numerical calculations do not a priori assume a relation between $N_{\mathrm{H}_{\mathrm{I}}}$ and $n_{e}$, we must assume one in order to make use of the analytic approximation in Equation (7). The approximation curves in Figure 1 can be reproduced by taking $n_{e}=1.4 \times$ $10^{-3} \mathrm{~cm}^{-3}\left(N_{\mathrm{H}} / 10^{17.2} \mathrm{~cm}^{-2}\right)^{2 / 3}\left(\Gamma_{\mathrm{HI}} / 10^{-12} \mathrm{~s}^{-1}\right)^{2 / 3}$. This relation is approximately derived under the assumptions of Jeans length thickness of the absorbers and optically thin photoionization equilibrium at $T=2 \times 10^{4} \mathrm{~K}$. Figure 1 shows that $A=0.15$ and $B=0.2$ give a good fit to our numerical results for a wide range of external illuminating spectra. The fitting formula has the exact optically thin limit; the asymptotic divergence from the numerical results as $N_{\mathrm{H}_{\mathrm{I}}} \rightarrow \infty$ is unimportant as most of the He II opacity arises in systems with $\tau_{\mathrm{H}_{\mathrm{I}}} \lesssim 1$. Although we have assumed specific (but varying) spectral shapes in determining the fitting parameters $A$ and $B$, the Fardal et al. (1998) derivation of the functional form in Equation (7) illustrates how the relation between $N_{\mathrm{He} \text { II }}$ and $N_{\mathrm{HI}}$ depends principally on the photoionization rates $\Gamma_{\mathrm{HI}}$ and $\Gamma_{\mathrm{He}}$. The relation should therefore hold well in general.

Obtaining a physically motivated analytic approximation to $\zeta \equiv N_{\mathrm{He}} / N_{\mathrm{HI}}$ is more difficult since $y_{\mathrm{He} \text { II }}$ is not readily known (for $\eta$, we know that $x_{\mathrm{H} \text { II }} \approx y_{\mathrm{III}} \approx 1$ in the almost completely ionized case, so that the ionized fractions do not appear explicitly in Equations (6) and (7)). Because the ionization potential of $\mathrm{He} \mathrm{I}$ is relatively close to that of $\mathrm{HI}$, their ionization states are similar and since helium is less abundant by a factor of 12 by number, He I should contribute relatively little to the ionizing opacity. This intuition is supported by the right panel of Figure 1, which shows that $\zeta$ versus $N_{\mathrm{HI}}$ is $\ll 1$ for illuminating spectra considered. After hardening by IGM filtering, both star-forming galaxies and quasars are expected to produce roughly flat spectra between the $\mathrm{HI}$ and $\mathrm{He}$ I ionization edges (Section 5), so that a representative case is $\alpha \approx 0$, yielding $\zeta \lesssim 10^{-3}$. We therefore approximate $\zeta=0$ in our cosmological calculations and verify using toy cases of constant $\zeta$ that this is justified in Section 6.

\section{RECOMBINATION EMISSION}

\subsection{Cosmological Emissivity}

The cosmic absorbers not only act as sinks but also as sources of ionizing radiation as a certain fraction of ionizations are followed by the re-emission of other ionizing photons via recombinations (Fardal \& Shull 1993; Haardt \& Madau 1996; Fardal et al. 1998). This recombination emission must be taken into account because it may boost the photoionization rates and also since line re-emission can imprint significant narrow features in the ionizing background spectrum. Our approach to include this recombination contribution is based on the self-consistent numerical calculations of recombination emission from individual absorbers using the code outlined in the previous section and detailed in Appendix A. This again differs from Haardt \& Madau (1996), who used an analytical escape probability formalism and made the assumption of a constant source function within the absorbers (which breaks down in the very optically thick systems), and from the treatment of Fardal et al. (1998) and thus provides a check of these results.

For each recombination process of interest, we calculate the emergent specific intensity $I_{v}^{\text {rec }}\left(N_{\mathrm{H}_{\mathrm{I}}}\right)$ owing to the process given the external illuminating spectrum numerically using our photoionization code. The cosmological recombination emissivity for this process is then an average over the column density distribution:

$$
\epsilon_{\nu}^{\mathrm{rec}}=4 \pi \frac{d z}{d l} \int_{0}^{\infty} d N_{\mathrm{H} \mathrm{I}} \frac{\partial^{2} N}{\partial z \partial N_{\mathrm{H}}} I_{\nu}^{\mathrm{rec}}\left(N_{\mathrm{H}_{\mathrm{I}}}\right) .
$$


Since in general $I_{v}^{\mathrm{rec}}\left(N_{\mathrm{H}}\right)$ depends on the spectrum of the ionizing background, which is not known a priori and evolves at each step in the redshift integration, it is again necessary to obtain an analytical approximation for this function that scales appropriately with the external background, as it is not practical to perform self-consistent numerical calculations in the cosmological solution. We develop these analytical approximations in Appendix B for each recombination process of interest.

\subsection{Recombination Processes}

For hydrogen, the only ionizing process is direct recombination to the ground state, which produces a 1 Ryd Hi LyC photon. For helium, both recombinations to $\mathrm{He}$ II and to $\mathrm{He} \mathrm{I}$ can in principle produce ionizing photons. In cosmological conditions, He I plays a negligible role (Sections 3.2 and 6; Haardt \& Madau 1996) and we will ignore it in our re-emission calculations. Three permitted He II recombination channels lead to the re-emission of ionizing photons: He II LyC recombinations directly to the ground state, indirect recombinations leading to He II Ly $\alpha$ emission, and recombinations to the $n=2$ excited level resulting in Balmer continuum (BalC) photons. These respectively give photons of energy 4, 3, and 1 Ryd. Higher He II Lyman-series photons could also produce ionizing photons, but we assume case B conditions in which these are ultimately degraded into lower energy photons, the only ones of which that can ionize hydrogen being $\mathrm{He}$ II Ly $\alpha$. We do not include forbidden two-photon recombination processes as these are energetically subdominant and do not result in distinctive emission features.

In calculating the contribution of re-emission to the photoionization rates, it is important to model the finite width of the recombination lines. If $\mathrm{H} \mathrm{I} \mathrm{LyC}$ re-emission is incorrectly modeled as a $\delta$ function, the re-emission photons are immediately redshifted below the $\mathrm{H}$ i ionization edge and are lost as contributors to the ionizing background. For continuum recombinations, the line profile is well approximated by

$$
\phi_{\mathrm{rec}}(v)=\frac{\left(v / v_{\mathrm{rec}}\right)^{-1} \exp (-h v / k T)}{\Gamma\left(0, h v_{\mathrm{rec}} / k T\right)} \frac{\theta\left(v-v_{\mathrm{rec}}\right)}{v_{\mathrm{rec}}},
$$

where $T$ is the temperature of the gas, and $\theta(\Delta v)$ is the Heaviside function which is 1 for $\Delta v \geqslant 0$ and 0 otherwise (Appendix E). Electrons in gas at higher temperature tend to have large kinetic energy and so give rise to higher energy recombination photons that take longer to redshift below the ionization edge. In Appendix E, we show that broadening owing to thermal and peculiar motion is negligible relative the width of the profile in Equation (9). For Ly $\alpha$ emission, either by $\mathrm{H}$ I or He II, a $\delta$ function profile $\phi_{\mathrm{H}_{\mathrm{I}} / \mathrm{He}{ }_{\mathrm{ILy}} \alpha}(v)=\delta\left(v-v_{\left.\mathrm{H} \mathrm{I} / \mathrm{He}{ }_{\text {ILy } \alpha}\right)}\right)$ is however appropriate because of the narrow intrinsic line width (much smaller than the mean free path by which photons are redshifted before being reabsorbed) and its distance from the ionization edges. While resonant scattering radiative transfer effects can broaden Ly $\alpha$ emission lines by $\sim 10-1000 \mathrm{~km} \mathrm{~s}^{-1}$ (e.g., Neufeld 1990; Zheng \& Miralda-Escudé 2002; Dijkstra et al. 2006; Verhamme et al. 2006), this width is negligible in comparison to the cosmological redshift broadening.

The analytical approximations for the recombination emission from individual absorbers are compared to the full numerical calculations in Figure 2 for the ionizing processes. In all cases, $\Gamma_{\mathrm{H}_{\mathrm{I}}}^{\mathrm{rec}} / \Gamma_{\mathrm{H}_{\mathrm{I}}}^{\mathrm{ext}}$ (where we define $\Gamma_{\mathrm{H}_{\mathrm{I}}}^{\mathrm{rec}}\left(N_{\mathrm{H}_{\mathrm{I}}}\right) \equiv$ $\left.4 \pi \int_{\nu_{\mathrm{HI}}}^{\infty} d v /(h v) I_{v}^{\mathrm{rec}}\left(N_{\mathrm{HI}}\right) \sigma_{\mathrm{HI}}(v)\right)$ is maximum for $\mathrm{HI} \mathrm{LyC}$ re-emission, as expected since hydrogen recombinations are more frequent owing to its greater abundance, and these recombination photons have the largest photoionization cross section, and is equal to about $10 \%$. The helium recombination processes all contribute at the $10^{-3}$ level or less. Note, however, that He II LyC re-emission will contribute more significantly to the He II ionizing background and that processes which contribute negligibly to the photoionization rates can still imprint important narrow features in the background spectrum that can be important for metal line studies. The agreement between the numerical calculations and analytical approximations is generally good and the approximations scale well for different spectral indices. Discrepancies of a factor of a few exist over some column density intervals, particularly for the He II BalC and He II Lyman continuum (LyC) processes. These processes are complex in their details that depend on the non-monotonic relative ionization of hydrogen and helium (Figure 1) but their contributions are nevertheless reasonably well captured and fortunately subdominant to the photoionization rates. In contrast, the dominant contribution of re-emission to the hydrogen ionizing background, $\mathrm{H}$ I LyC emission, involves only hydrogen and is accurately and robustly approximated.

Figure 3 compares the analytical approximation for $\mathrm{H}$ I $\mathrm{Ly} \alpha$ approximation to the full numerical solutions. In this case, both the optically thin and optically thick limits are accurately captured, resulting in an excellent approximation at all column densities that scales correctly with the external illuminating spectrum.

\section{EMPIRICALLY CALIBRATED SPECTRA}

\subsection{Quasar and Stellar Emissivities}

Having established efficient approximations for the radiative transfer within individual absorbers (Sections 3 and 4), we proceed to include these in the solution of the cosmological radiative transfer problem (Section 2). Our prescriptions for the sources of ionizing radiation are based on the empirical constraints obtained in Faucher-Giguère et al. (2008d, 2008a, 2008b). Note, however, that these prescriptions can easily be modified to accommodate further constraints: our numerical code can compute the ionizing background for arbitrary input emissivities. We explore variations about these fiducial parameters in Section 6. Here, we consider two dominant known sources of ionizing radiation: quasars and star-forming galaxies.

For the quasar emissivity, we use the quasar luminosity function of Hopkins et al. (2007) based on a large set of observed quasar luminosity functions in the infrared, optical, soft and hard $\mathrm{X}$-rays, as well as emission line measurements. Denoting by $\epsilon_{B}$ the emissivity at $4400 \AA$ and assuming $\left.L_{B} \equiv v L_{v}\right|_{4400 \AA}$,

$$
\epsilon_{B}^{\mathrm{QSO}, \mathrm{com}}=\int_{0}^{\infty} d L_{B} \frac{d \phi}{d L_{B}} \frac{L_{B}}{\left.\nu\right|_{4400 \AA}},
$$

where $d \phi / d L_{B}$ is the $B$-band luminosity function in comoving units. The emissivity shortward of $4400 \AA$ is calculated assuming that quasars have a spectral index $\alpha=0.3$ at $2500-4400 \AA$, 0.8 at 1050-2500 $\AA$ (Madau et al. 1999), and $\alpha_{\mathrm{QSO}}$ shortward of $1050 \AA$. In order to match the total H i photoionization rate measured from the Ly $\alpha$ forest and to account for uncertainties in converting from the emissivity at $4400 \AA$ to the photoionization rate, we allow this emissivity to be normalized by a constant factor (see Section 5.2). In our fiducial model, $\alpha_{\mathrm{QSO}}=1.6$ (Telfer et al. 2002) but note that other studies have found both softer 

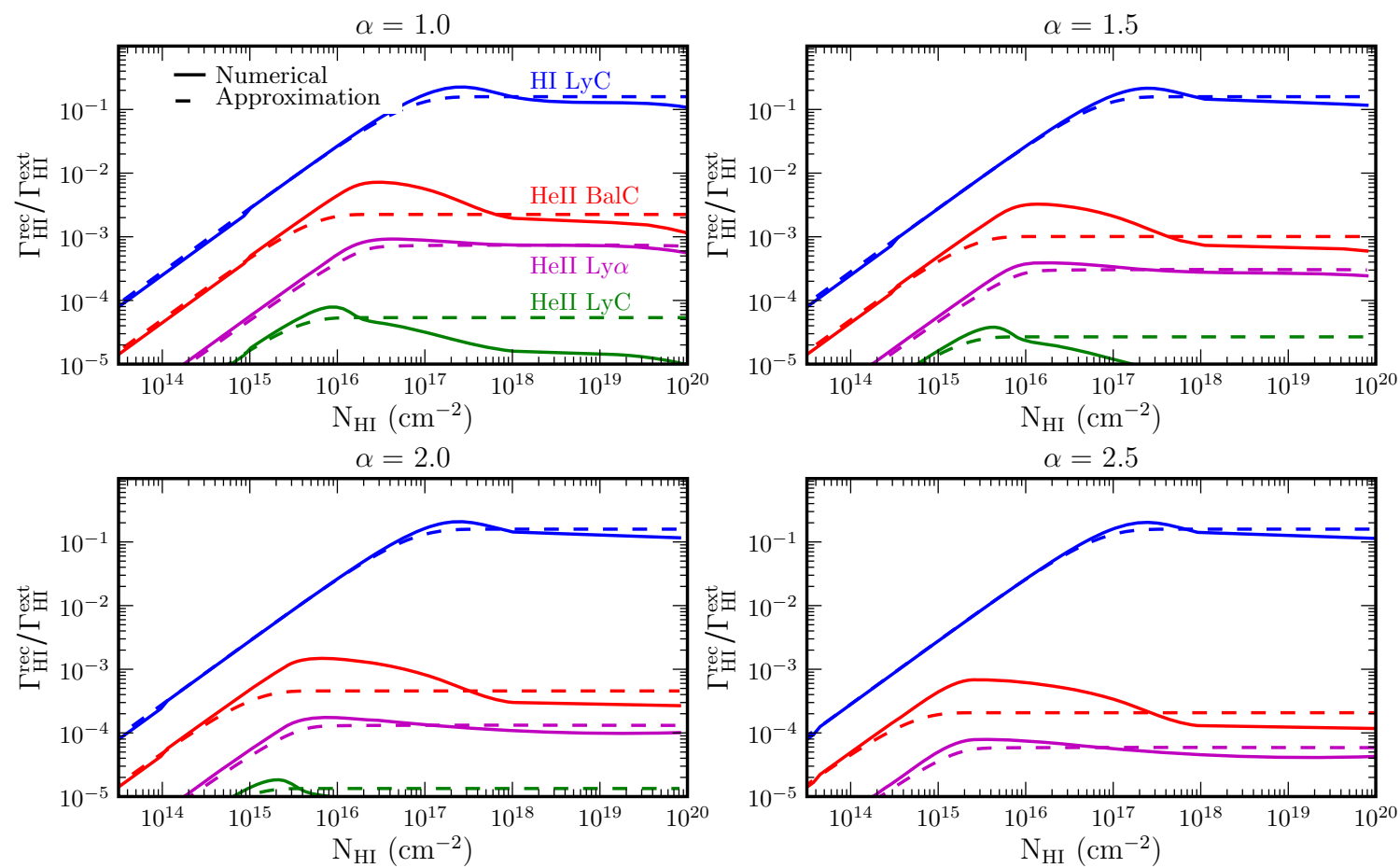

Figure 2. Ratio of the H I photoionization rate outside an individual absorber that is contributed by different recombination processes (HI LyC in blue, He II BalC in red, $\mathrm{He}$ II $\mathrm{Ly} \alpha$ in magenta, $\mathrm{He}$ II $\mathrm{LyC}$ in green) to the external photoionization rate. The solid curves show full numerical integrations over photoionized slabs (Equation (B2)) and the dashed ones show analytical approximations based on the optically thin limit (Equation (B3)) and saturation in the optically thick regime described in Section B.2. External power-law spectra $J_{v}^{\infty}=10^{-21} \mathrm{erg} \mathrm{s}^{-1} \mathrm{~cm}^{-2} \mathrm{~Hz}^{-1} \mathrm{sr}^{-1}\left(\nu / \nu_{\mathrm{HI}}\right)^{-\alpha}$ with $\alpha=1.0,1.5,2.0$, and 2.5, suppressed by a factor of 10 above the He II ionization edge, are assumed in the different panels.

(A color version of this figure is available in the online journal.)

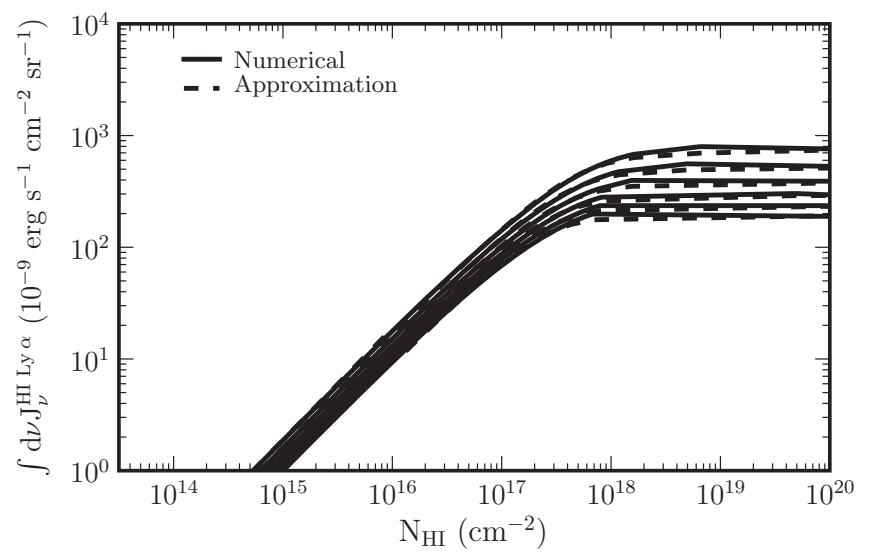

Figure 3. Frequency integral of $\mathrm{HI}_{\mathrm{I}} \mathrm{Ly} \alpha$ re-emission as a function of $\mathrm{HI}$ column density. The solid curves show full numerical photoionization calculations, and the dashed ones show analytical approximations based on Equation (B8). From the bottom up, power-law external spectra $J_{v}^{\infty}=$ $10^{-21} \mathrm{erg} \mathrm{s}^{-1} \mathrm{~cm}^{-2} \mathrm{~Hz}^{-1} \mathrm{sr}^{-1}\left(\nu / \nu_{\mathrm{HI}}\right)^{-\alpha}$ with $\alpha=0.0,0.5,1.0,1.5,2.0$, and 2.5 , suppressed by a factor of 10 above the He II ionization edge, are assumed.

and harder spectra, with a significant variance about the mean (e.g., Zheng et al. 1997; Scott et al. 2004).

For the stellar emissivity, we assume that the emissivity is proportional to the star formation rate density,

$$
\epsilon_{v}^{\star, \text { com }}=K \dot{\rho}_{\star}^{\text {com }},
$$

with the observationally calibrated proportionality constant accounting for the efficiency of conversion of mass into ionizing photons. We use the theoretical star formation history of
Hernquist \& Springel (2003) developed from a combination of hydrodynamical simulations (Springel \& Hernquist 2003) and simple analytical arguments. In Faucher-Giguère et al. (2008b), we found that this model provides a better fit at high redshifts to the opacity of the Ly $\alpha$ forest over $z=2-4.2$, is easier to reconcile with hydrogen reionization completing by $z=6$, and is in better agreement with the rate of long gamma-ray bursts observed by Swift than many of the existing measurements based on galaxy surveys, among which there is still a wide dispersion. We use Equation (45) of Hernquist \& Springel (2003) to scale their fiducial model to the WMAP5 cosmology assumed in this work. We assume, motivated by the theoretical starburst calculations of Kewley et al. (2001), that star-forming galaxies have a spectral index $\alpha_{\star}=1$ between 1 and 4 Ryd. This model is applicable for the stellar populations calculated with the PEGASE code using the Clegg \& Middlemass (1987) atmosphere models for Wolf-Rayet stars. While different theoretical assumptions lead to significant variance in the 1-4 Ryd spectrum, this model provides the best observational match to the hard starburst spectra inferred by optical line diagnostics by Kewley et al. (2001). We assume that they effectively emit no harder photons, the theoretical calculations showing a break of several orders of magnitude at the He II ionization edge. In the UV spectrum redward of 1 Ryd, we take $\alpha_{\star}=0$, consistent with the Lyman break galaxies observed by Shapley et al. (2003). Finally, we assume that the stellar emissivity has a discontinuity of a factor of 4 at the Lyman limit. While this factor is neither well constrained empirically or observationally, it only affects our predicted spectra (normalized to the measured ionizing background) at energies less than 1 Ryd, which we do not attempt to accurately model in this work. 

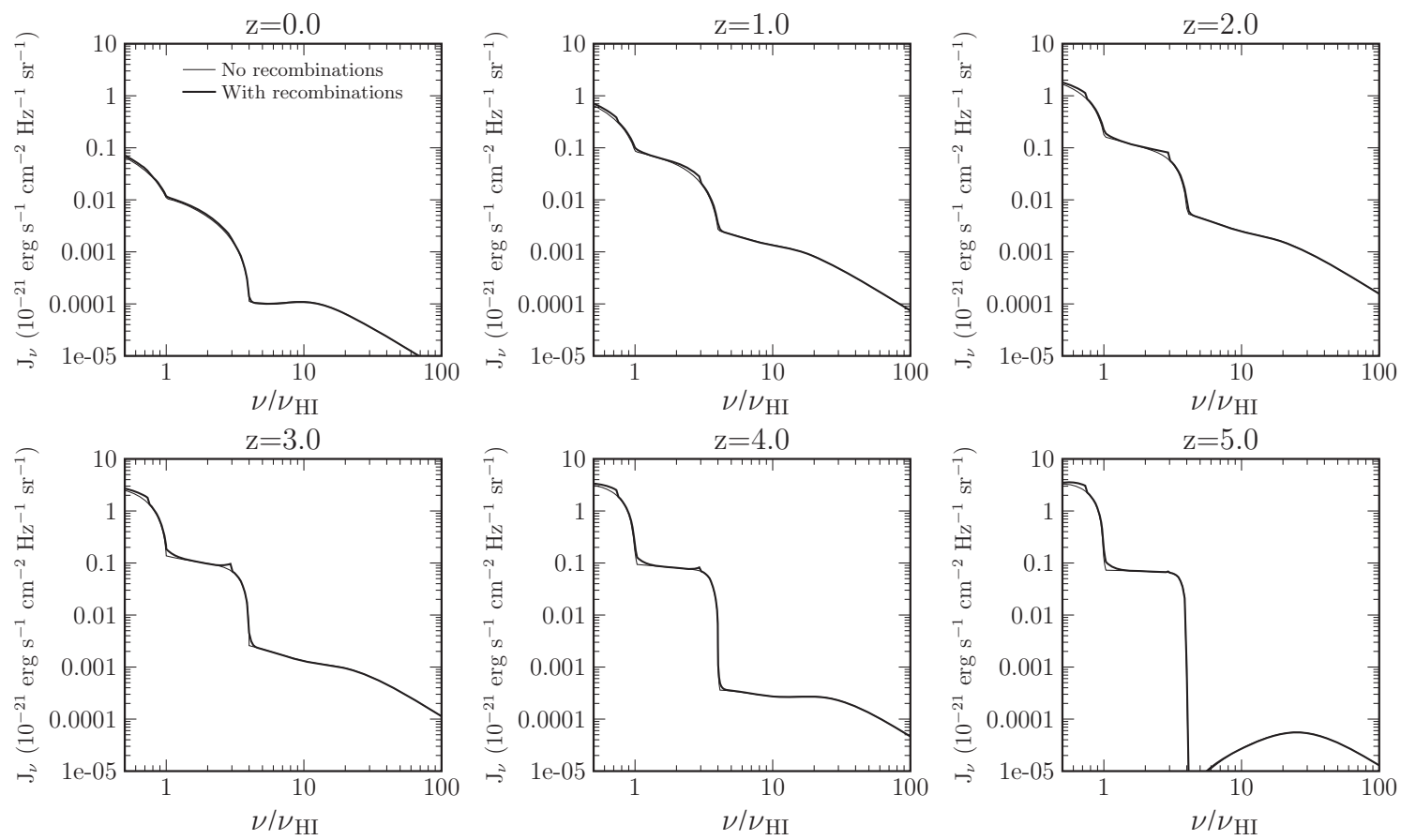

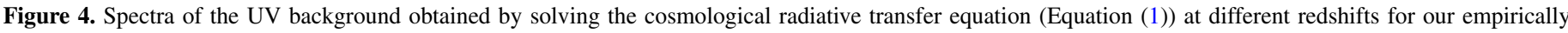

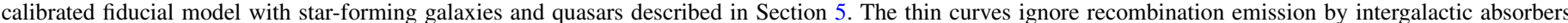

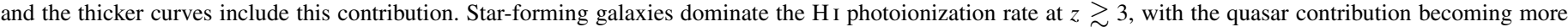

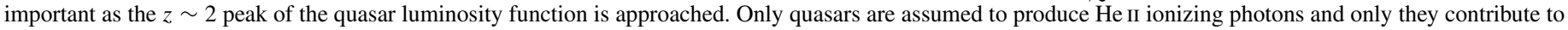
He II recombination processes. The integrated photoionization rates are given in Figure 5.

The emissivities are converted to proper units before being inserted in the solution to the cosmological radiative transfer solution in Equation (2) and the total emissivity is then $\epsilon_{v}(z)=$ $\epsilon_{v}^{\mathrm{QSO}}(z)+\epsilon_{v}^{\star}(z)+\epsilon_{v}^{\mathrm{rec}}(z)$.

\subsection{Results}

In Figure 4, we show the calculated cosmological UV background spectra at $z=0-5$ for the fiducial model above, with and without the recombination processes included. Since only quasars are assumed to contribute photons above the 4 Ryd He II ionization edge, only them contribute to the He II recombination lines and photoionization rate.

In Figure 5, we show the integrated photoionization rates of $\mathrm{HI}$ and He II, as well as the fractional contribution of recombination lines with respect to the total background including both stars and quasars. The quasar contribution to the $\mathrm{H}_{\mathrm{I}}$ ionizing background increases toward $z \sim 2$ as the peak of the quasar luminosity function is approached; the $z \gtrsim 3$ photoionization rate is dominated by stellar emission. The fractional recombination contribution to the $\mathrm{H}$ i photoionization rate ranges from $5 \%$ to $17 \%$ over the interval $z=0-6$, significantly smaller than the $\alpha_{\mathrm{H}, \mathrm{n}=1}(T) /\left.\alpha_{\mathrm{HI}}^{\mathrm{A}}(T)\right|_{T=20,000 \mathrm{~K}} \approx 48 \%$ fraction of $\mathrm{H}$ I recombinations that are directly to the ground state. The relatively small contribution of recombinations to the ionizing background owes to a combination of the saturation of re-emission in optically thick systems (Figure 2), leakage of the re-emitted photons at the ionizing edge, and the frequency dependence of the photoionization cross section (Section 6.2 and Appendix C).

The fractional contribution of He II recombinations to the He II photoionization rate is also relatively small for the same reason, but is more difficult to calculate accurately at redshifts $z \gtrsim 4$ in our model. In order to obtain an accurate value, in addition to the $\mathrm{He}$ II $\mathrm{LyC}$ re-emission line to be well resolved on the computational frequency grid, the mean free path of He II ionizing photons must also be well resolved by the redshift grid. In our calculation, quasars produce a negligible and rapidly dropping He II photoionization rate at $z \gtrsim 4$ while star-forming galaxies maintain a roughly constant $\mathrm{H}$ I photoionization rate. In these conditions, the ratio $\eta=N_{\mathrm{He} \text { II }} / N_{\mathrm{HI}}$ tends to infinity and the He II mean free path to zero, making it exceedingly difficult to resolve it. Fortunately, the total He II photoionization rate in this regime is so small that its fractional enhancement from recombinations is of little practical importance. Moreover, in this regime, He II reionization may well be still underway and the He II ionizing background consequently modified, as elaborated on in Section 7. In Figure 5, we indicate the portion of poor convergence by a dashed curve segment; the turnover of $\Gamma_{\mathrm{He} \text { II }}^{\text {withec }} / \Gamma_{\mathrm{He} \text { II }}^{\text {norec }}$ around $z \sim 5$ is likely an artifact and we in fact expect it to continue to increase slightly toward higher redshifts owing to the reduced leakage (Section 6.2).

The total $\mathrm{H}_{\mathrm{I}}$ photoionization rate matches the value $\Gamma_{\mathrm{HI}_{\mathrm{I}}}=$ $(0.5 \pm 0.1) \times 10^{-12} \mathrm{~s}^{-1}$ derived from the Ly $\alpha$ forest at $z=2-4.2$, subject to the constraint that quasars must contribute a large fraction near their peak (Faucher-Giguère et al. 2008a, 2008b). This was done by normalizing the nominal quasar contribution (Section 5.1) by a factor of 0.36 and normalizing the stellar contribution so as to provide the rest of the ionizing photons. The renormalization of the quasar contribution can be justified by uncertainties in the mean free path of $\mathrm{H}_{\mathrm{I}}$ ionizing photons (a direct product of the prescribed $\mathrm{H}$ I column density distribution), in their escape fraction, and in the quasar spectral template (see discussion in Faucher-Giguère et al. 2008b). These uncertain factors enter in the conversion from the quasar luminosity to the photoionization rate. Since we wish to reproduce the more robustly constrained photoionization rate measured from the Ly $\alpha$ forest, we adjust the normalization accordingly. 

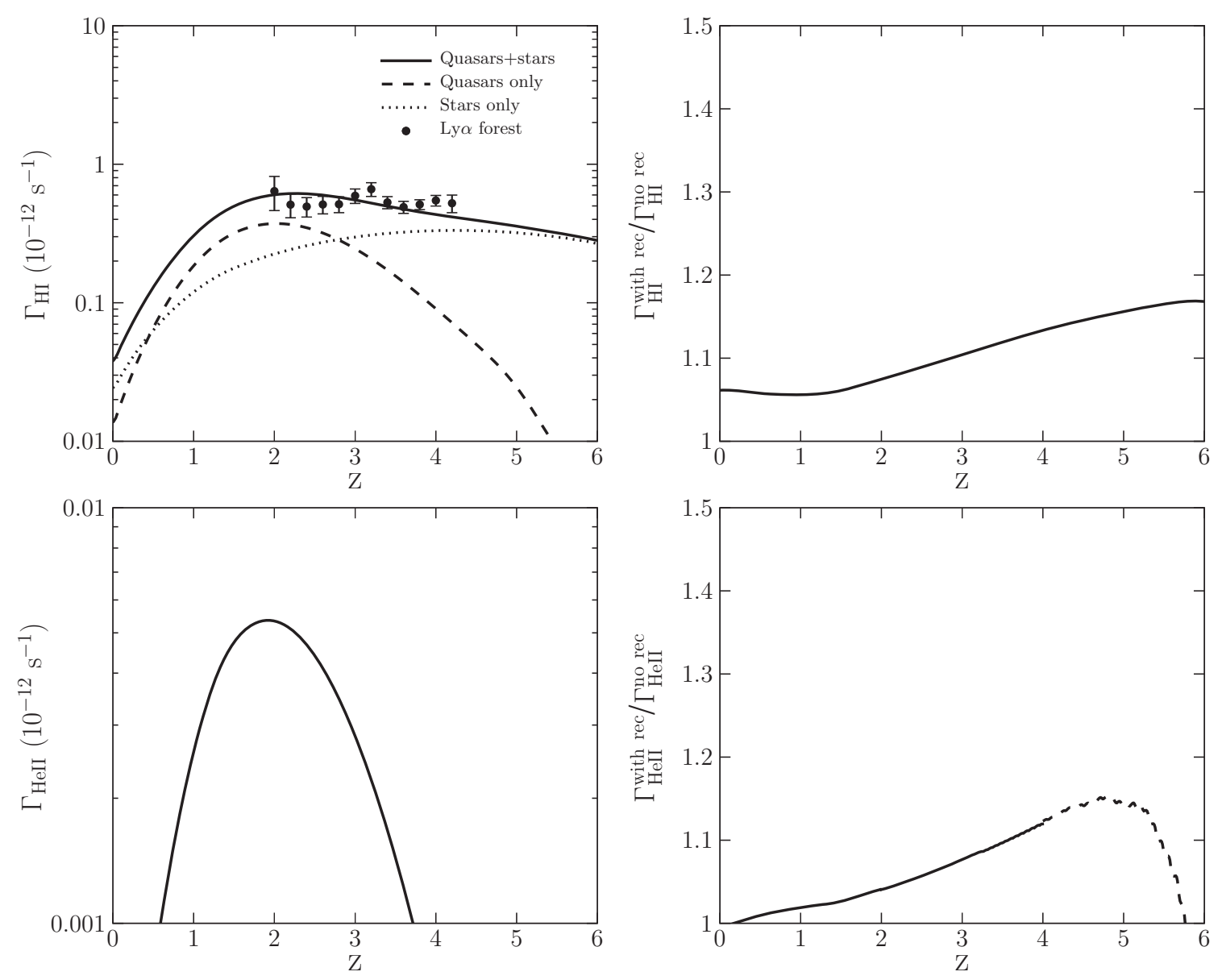

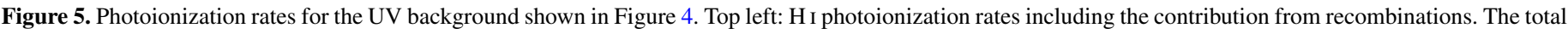

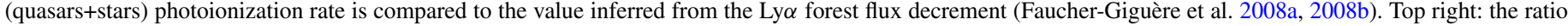

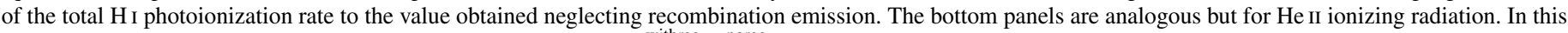

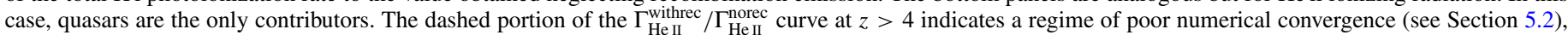
and the turnover at $z \sim 5$ is likely an artifact.

Although our calculations are normalized to match the hydrogen photoionization rate measured from the Ly $\alpha$ forest, it is important to emphasize that this measurement and hence the normalization of the spectra calculated here are somewhat uncertain. The measurement was obtained using the flux decrement method (e.g., Rauch et al. 1997), in which we solve for the value of $\Gamma_{\mathrm{H}_{\mathrm{I}}}$ needed to produce the measured mean transmission of the Ly $\alpha$ forest. Two important sources of systematic uncertainty are the assumed IGM temperature (since the flux decrement constrains only the combination $\left.\Gamma_{\mathrm{H}_{\mathrm{I}}} / \alpha_{\mathrm{H}_{\mathrm{I}}}^{A}(T)\right)$ and the gas density distribution (whose details depend on the cosmological parameters and thermal history). Another potential worry is that the measured Ly $\alpha$ forest mean transmission may be increasingly biased high toward high redshifts (inducing a redshift-dependent error) as the continuum level is increasingly absorbed and difficult to estimate directly. We have however quantified and corrected for this effect in our measurement (Faucher-Giguère et al. 2008d), and so it should not affect our results. In the end, we expect the measured $\Gamma_{\mathrm{H}_{\mathrm{I}}}$ to be accurate within a factor $\sim 2$, with the possible errors mostly systematic and weakly dependent on redshift. For a more exhaustive discussion of the uncertainties of the measured $\Gamma_{\mathrm{H}}$, see Section 3 of Faucher-Giguère et al. (2008b).

Finally, we must also note that for precise work with hydrodynamical simulations, the simulated Ly $\alpha$ forest mean trans- mission should always be compared with the measured value and the photoionization rates renormalized if necessary. In fact, even if the correct ionizing background (with the correct normalization) is prescribed, the simulated mean transmission may be slightly off if, for example, the temperature of the IGM is incorrect reproduced. This is particularly likely to occur if the effects of H I and He II reionization (see Section 7) are not explicitly modeled.

\section{DEPENDENCES ON INPUT PARAMETERS}

Even after fixing the stellar and quasar emissivities for our fiducial model, the spectrum calculations depend on a number of parameters. It is useful to investigate how the calculated spectrum and its integrals depend on these as their values are only known to limited precision. This also provides a physical understanding of the shape of the calculated spectra. We begin by considering the dependences of the overall ionizing background spectrum in Section 6.1 and focus on the recombination contribution in Section 6.2.

\subsection{Overall Spectrum}

In Figure 6, we show how the spectrum changes when the constant ratio $\zeta=N_{\mathrm{He}} / N_{\mathrm{HI}}$, the H I column density distribution power-law index $\beta$, and the stellar and quasar spectral indices 

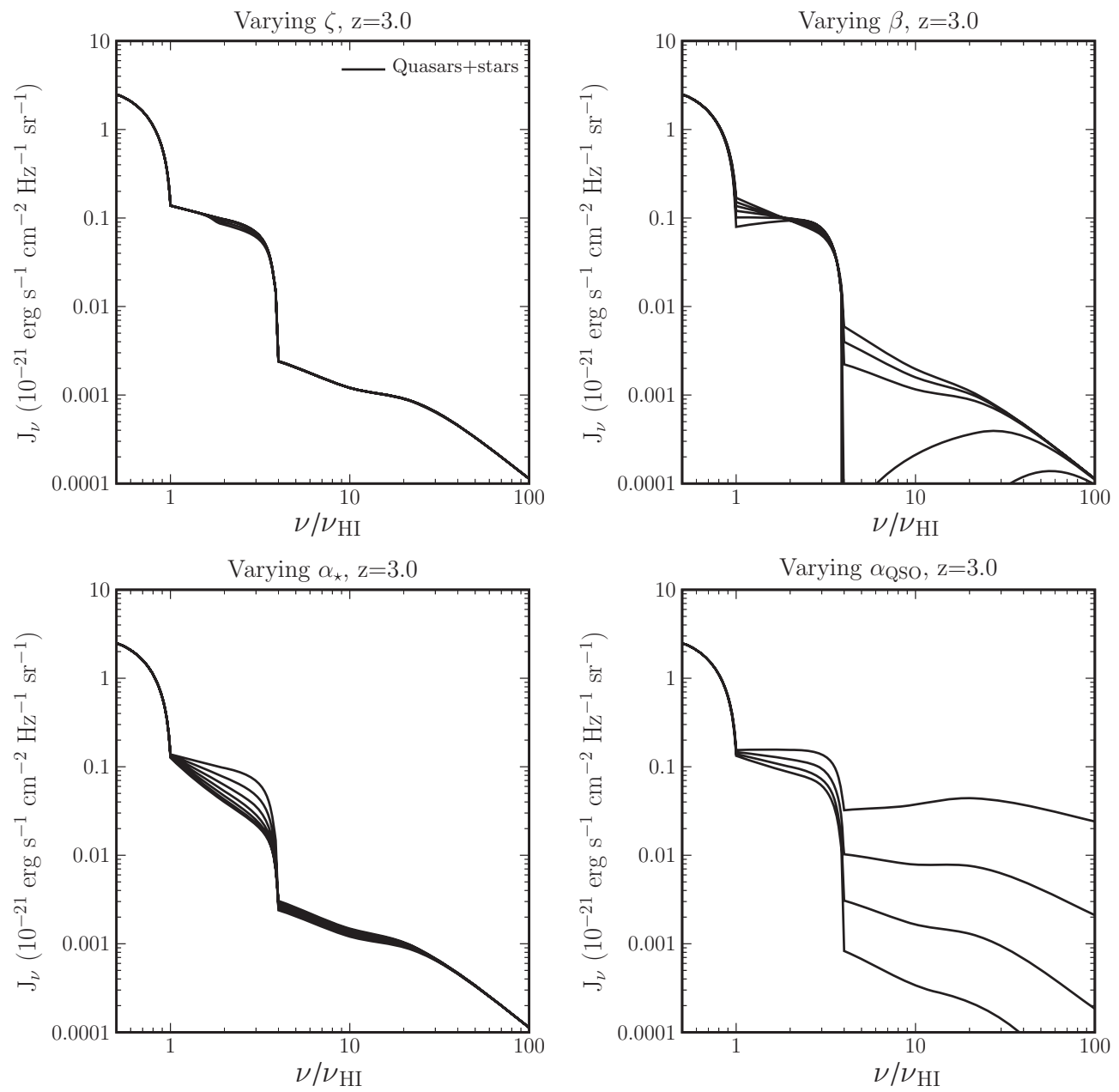

Figure 6. Effects of parameters on the calculated spectrum. Top left: varying the constant ratio $\zeta=N_{\mathrm{He}} / N_{\mathrm{HI}}=0,0.001,0.005,0.01,0.05$, and 0.1 . Top right: varying the H I column density distribution power-law index $\beta=1.2,1.3,1.4,1.5,1.6$ and 1.7. Bottom left: varying the stellar spectral index $\alpha_{\star}=1.0,1.5,2.0,2.5,3.0,3.5$, and 4.0. Bottom right: varying the quasar spectral index $\alpha_{\mathrm{QSO}}=0.5,1.0,1.5$, and 2.0. In each panel, the curves correspond to these values from the top to the bottom. For realistic values of $\zeta$ (Section 3.2 and Figure 1) for a background spectrum arising from stars and quasars, the effect of He I is small. The column density distribution power law $\beta$ determines the spectral hardening just shortward of the ionization edges. The stellar and quasar spectral indices determine the spectral slope of the background. For fixed emissivity at the Lyman limit, the stellar spectral index has only a modest effect on the amplitude of the spectrum because it is truncated at 4 Ryd. The quasar spectral index, assumed to extend to infinity, has a more drastic overall impact toward high energies. Recombination emission has been omitted for clarity of presentation.

$\alpha_{\star}$ and $\alpha_{\mathrm{QSO}}$ are individually varied. In each case, all other parameters are fixed to the fiducial model of the previous section. Even for a constant ratio $\zeta=0.1$, a factor more than 100 times that expected in our fiducial calculation (Section 3.2), He I absorbs only a very small fraction of the spectrum shortward of its ionization edge. It is therefore a good approximation to neglect it in our cosmological calculations. The H I column density distribution power-law index $\beta$ determines the spectral hardening just above the ionization edges following $\alpha \rightarrow$ $\alpha-3(\beta-1)$ (Appendix D) as well as the depth of the absorption edges. Note that the depth of the He II absorption edge is more sensitive to $\beta$; this arises because the column density distribution is normalized to the abundance of Hi Lyman limit systems (Section 2.2) so that it is fixed in these calculations while the abundance of the He II Lyman limit systems varies. The stellar and quasar spectral indices simply determine the spectral slopes of the background prior to hardening. For fixed emissivity at the Lyman limit, the stellar spectral index has only a modest effect on the amplitude of the spectrum because it is truncated at $4 \mathrm{Ryd}$. The quasar spectral index, assumed to extend to infinity, has a more drastic overall impact toward high energies: as $v \rightarrow \infty$ and spectral hardening becomes negligible, different spectral

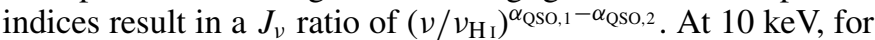
example, this ratio is 735 for $\alpha_{\mathrm{QSO}, 1}=1.5$ and $\alpha_{\mathrm{QSO}, 2}=0.5$; redshifted from $z=2$ to $z=0$, this falls in the bandpass of $\mathrm{X}$-ray observatories such as Chandra and XMM-Newton. As most ( $\gtrsim 80 \%$ ) of the soft X-ray background has already been resolved into active galactic nuclei (e.g., Hasinger et al. 2005), the X-ray background is a powerful probe of the high-energy quasar spectral energy distribution, although a proper analysis requires the inclusion of obscured quasars, which we do not explicitly consider in this work (e.g., Gilli et al. 2007).

In Figure 7, we explore how the hydrogen photoionization rate is affected by the redshift evolution of the column density distribution. In the left panel, we vary the redshift at which the redshift evolution of the column density distribution flattens (Section 2.2) from $z_{\text {low }}=0$ to $z_{\text {low }}=2$. Interestingly, this has a minimal impact on the redshift evolution of the photoionization rate even if it does significantly change the mean free path of $\mathrm{H}_{\mathrm{I}}$ ionizing photons at these redshifts. This is easily understood as a consequence of the fact that the universe effectively becomes transparent at a "breakthrough" redshift $z_{\mathrm{bt}} \sim 2$ (Madau et al. 

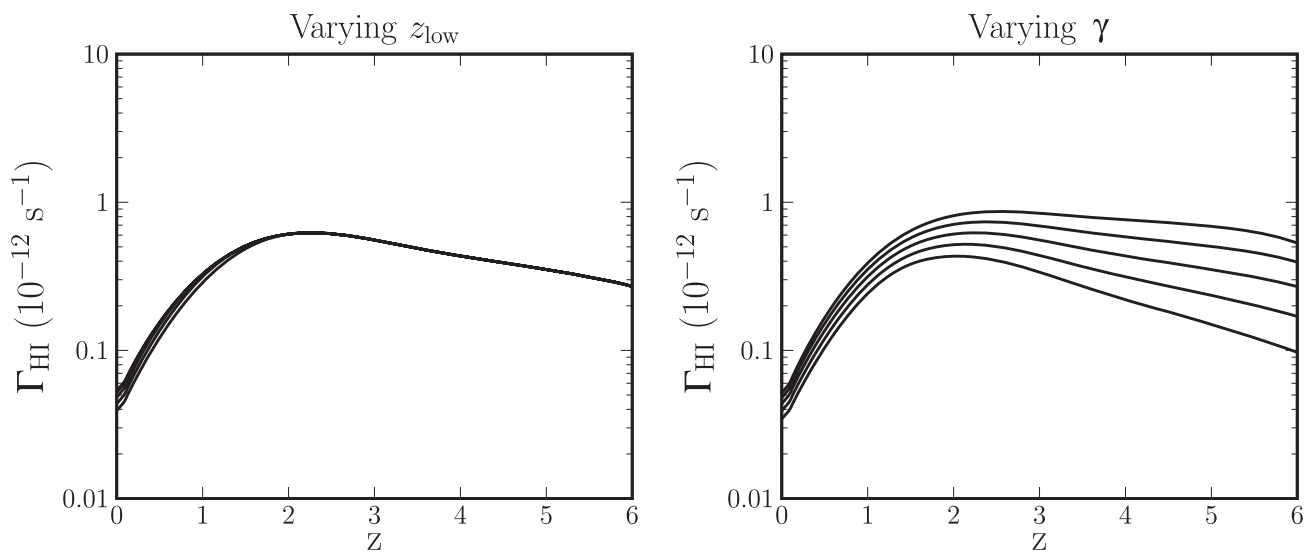

Figure 7. Effects of the redshift evolution of the $\mathrm{HI}$ column density distribution on the hydrogen photoionization rate. Left: varying the redshift at which the column density distribution transitions to relatively flat evolution $z_{\text {low }}=0,0.5,1.0,1.5,2.0$ (bottom up). Right: varying the power-law index of redshift evolution $\gamma=1.0,1.25,1.5,1.75$, and 2.0 (top-bottom) of the column density distribution at $z>z_{\text {low }}$. Varying $z_{\text {low }}$ at $z \leqslant 2$ has little impact on $\Gamma_{\mathrm{HI}}$ since at low redshift the $\mathrm{HI}$ ionizing mean free path is sufficiently large that the spectral intensity is limited by the cosmological horizon. The high-redshift $\Gamma_{\mathrm{HI}}$ declines more rapidly with more a rapid increase in the abundance of absorbers with redshift, or large $\gamma$, translating into a more rapidly diminishing mean free path.

1999), below which the mean free path becomes so large the local ionizing background is not limited by the latter but by the cosmological horizon. As shown in the right panel, the highredshift $\Gamma_{\mathrm{H}_{\mathrm{I}}}$ declines more rapidly with more a rapid increase in the abundance of absorbers with redshift, or large $\gamma$, translating into a more rapidly diminishing mean free path. At present, although more than a decade old, the best constraints on the abundance of the Lyman limit systems (Storrie-Lombardi et al. 1994, 1996; Stengler-Larrea et al. 1995) are relatively loose and mostly nonexistent beyond $z=4$. As future measurements refine these and push toward higher redshifts, it is possible that these will give more credence to one of the alternative values of $\gamma$ plotted here.

\subsection{Recombination Contribution}

The contribution of recombinations to the photoionization rates, $\Gamma^{\text {withrec }} / \Gamma^{\text {norec }}$, is a subtle question as it depends on several factors. It not only depends on the number of re-emitted ionizing photons integrated over the distribution of absorbers (Section 4) but also crucially on the energy at which these photons are reemitted as well as on their redshifted energy at the point of evaluation of the photoionization rate.

The LyC recombination line processes, most important for the boosting the ionization rates, reemit ionizing photons just above the ionization edges of H I or He II. Since the ionizing background at a given point is sourced along its past light cone, its photons have generally redshifted slightly from their emission energy. As a result, many recombination photons with initial energy just above their corresponding ionization edges quickly redshift below these edges and are lost as contributors to the ionization rates. The fraction of ionizing recombination photons lost in this way depends on two factors: (1) the recombination line profile which determines how far above the ionization edge an ionizing photon is re-emitted and (2) the mean free path of ionizing photons which determines how long the photons have to redshift before they are absorbed. In the limit of a mean free path of zero length, the recombination photons cannot redshift before they are reabsorbed and no photons are lost; as the mean free path increases, more photons leak out of the ionizing range. Similarly, a narrow line profile concentrates the recombination photons just above the ionization edges, leading to a high probability of leakage, while a wider one allows them to remain longer in the ionizing range. Since the mean free path of ionizing photons is determined by the column density distribution and the recombination line width is determined by the temperature of the absorbers, these are important parameters for the recombination contribution. Finally, as recombination photons are re-emitted at energies above the ionization edges and subsequently redshift, the frequency dependence $\sigma_{i}(v) \propto v^{-3}$ of the photoionization cross section changes the weight they receive in the photoionization rates.

All these effects are self-consistently treated when solving the radiative transfer Equation (1). Figure 8 shows how much the $\mathrm{HI}$ photoionization rate is increased by recombination emission as a function of the normalization $C$ of the column density distribution, its power-law slope $\beta$ (see Equation (5)), and the temperature $T$ of the absorbers. We also show the case of the column density distribution assumed by Haardt \& Madau (1996), in which optically thin and optically thick absorbers have different redshift evolutions, leading to a redshift-dependent effective slope of the distribution (steeper at high redshifts).

Because the mean free path decreases with increasing abundance of Lyman limit systems (the normalization $C$ ), fewer recombination photons leak out of the ionizing range and so the ratio of the photoionization rate with and without recombinations, $\Gamma_{\mathrm{H}_{\mathrm{I}}}^{\text {withrec }} / \Gamma_{\mathrm{H} \text { I }}^{\text {norec }}$, increases. The ratio also increases with the steepness of the column density distribution since more recombinations occur in optically thin systems, from which practically all the recombination photons escape into the IGM, as opposed to in optically thick systems that trap a large fraction. Higher gas temperatures result in wider recombination lines so that fewer photons are lost owing to redshifting as well as a higher fraction of recombinations directly to the ground state $\left(\alpha_{\mathrm{H}, \mathrm{n}=1}(T) / \alpha_{\mathrm{H}_{\mathrm{I}}}^{\mathrm{A}}(T)\right.$; Appendix $\left.\mathrm{C}\right)$. A competing effect is that the recombination photons of high-temperature absorbers tend to have higher energies and receive less weight in the photoionization rate. The net effect is however relatively weak on $\Gamma_{\mathrm{HI}}^{\text {withrec }} / \Gamma_{\mathrm{H} \text { I }}^{\text {norec }}$ for the relevant temperature $T \sim 2.0 \times 10^{4} \mathrm{~K}$. Note that for any given set of parameters the ratio tends to increase toward higher redshifts since the mean free path is lower at the higher cosmological densities. This behavior is however not seen below the breakthrough redshift $z_{\text {bt }} \lesssim 2$, where the photoionization rate is limited by the cosmological horizon rather than by the mean free path. 

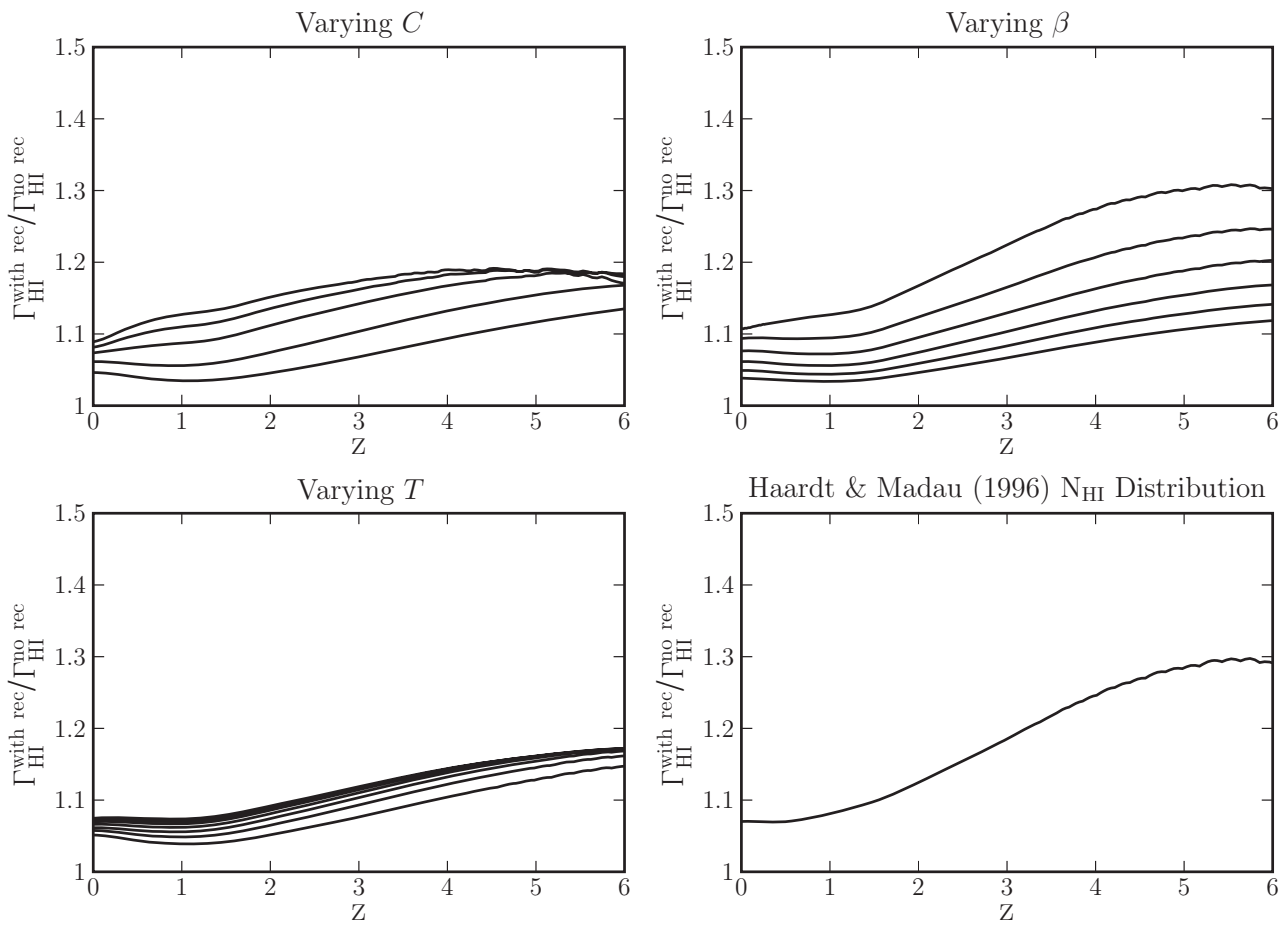

Figure 8. Dependences of the recombination contribution on H I photoionization rate. Shown are ratios of the total H i photoionization rate, including recombination emission, to the same calculation ignoring recombination emission. In all cases, the sources of the ionizing background are fixed to the fiducial model of Section 5, but we vary the parameters of the column density distribution and the temperature of the absorbers. Top left: $C=0.125,0.25,0.5,0.75$, and 1 from bottom up. Top right: $\beta=1.2,1.3,1.4,1.5,1.6$, and 1.7 from bottom up. Bottom left: $T=1.0,1.5,2.0,2.5,3.0,3.5$, and $4.0 \times 10^{4} \mathrm{~K}$ from bottom up. Bottom right: Haardt \& Madau (1996) column density distribution with different redshift evolutions in the optically thin and optically thick regimes.

In Appendix C, we develop a quantitative analytic model that captures and clarifies these effects and agrees well with the full numerical calculations presented here.

\section{REIONIZATION EVENTS}

The previous calculations have implicitly assumed that the universe is reionized in both $\mathrm{H}$ I and He II. This assumption is most evident in the case of $\mathrm{H}$, for which we have used a column density distribution measured from the $z \lesssim 4$ Ly $\alpha$ forest. The assumption creeps in for He II reionization during which there are large He II patches the inside which the He II photoionization rate is very low in comparison to within ionized bubbles. In each region, the mapping between $N_{\mathrm{HI}}$ and $N_{\mathrm{He} \text { II }}$ depends on the local spectrum and will in general be very inhomogeneous. The IGM opacity to He II ionizing photons will therefore be poorly approximated by using a globally averaged spectrum to map from $N_{\mathrm{HI}}$ to $N_{\mathrm{He}}$. In this work, we do not attempt to model $\mathrm{H}_{\mathrm{I}}$ reionization (and the likely simultaneous reionization of $\mathrm{He} \mathrm{I}$ ), which occurs at the limit of the present observational reach at $z>6$ (e.g., Fan et al. 2006a; Dunkley et al. 2009).

The reionization of He II was however likely delayed until the rise of the quasar luminosity function, at redshifts that are immediately accessible to observations and some understanding of its effects can be obtained by studying the ratio $\eta=N_{\mathrm{He} \text { II }} / N_{\mathrm{HI}}$. In fact, while stellar spectra are theoretically expected to have a strong break at the He II ionization edge and therefore have little impact on the He II ionization state, quasars have power-law far-UV spectra that extend well into the X-rays (Section 5.1). Theoretical calculations based on the quasar luminosity function in fact indicate that quasars can reionize He II by $z \sim 3-4$ (e.g., Sokasian et al. 2002; Wyithe \& Loeb 2003; Furlanetto \& Oh 2008; Faucher-Giguère et al. 2008b). A num- ber of lines of evidence, based on $\mathrm{HI}$ and He II Ly $\alpha$ forests as well as on the evolution of metal line ratios, also suggest that the IGM is undergoing changes that could be associated with $\mathrm{He}$ II reionization at these redshifts (for a review of these lines of evidence, see Faucher-Giguère et al. 2008d). While alternative candidate sources of $\mathrm{He}$ II reionization exist—such as possible He II ionizing emission from galaxies (e.g., Furlanetto \& Oh 2008), high-redshift X-rays (e.g., Oh 2001b; Ricotti \& Ostriker 2004), or thermal emission from shock heated gas (Miniati et al. 2004) — quasars are the best established and most likely. Large fluctuations observed in the He II ionizing background toward $z=3$, which can be explained by the small number density of bright objects, also lend support to the quasar hypothesis (Zheng et al. 2004; Shull et al. 2004; Bolton et al. 2006) and we will therefore concentrate on this scenario.

\subsection{The Ionizing Background During He II Reionization}

Recently, McQuinn et al. (2009) performed detailed radiative transfer simulations of He II reionization in large boxes up to 430 comoving Mpc on a side (for previous simpler treatments, analytic and numerical, see Sokasian et al. 2002; Bolton et al. 2004; Gleser et al. 2005; Paschos et al. 2007; Furlanetto \& Oh 2008; Bolton et al. 2009). These simulations used realistic models for the quasar sources based on the luminosity function of Hopkins et al. (2007) and with physically and empirically motivated prescriptions for the triggering of quasars in massive halos as well as of quasar light curves (see, e.g., Hopkins et al. 2005a, 2005b, 2006, 2008). A striking result of this work is the remarkable complexity of He II reionization, in particular of the He II ionizing radiation field, likely rendering the detailed resulting structure beyond analytic tractability. Nevertheless, some intuition on the spectrum and magnitude of 

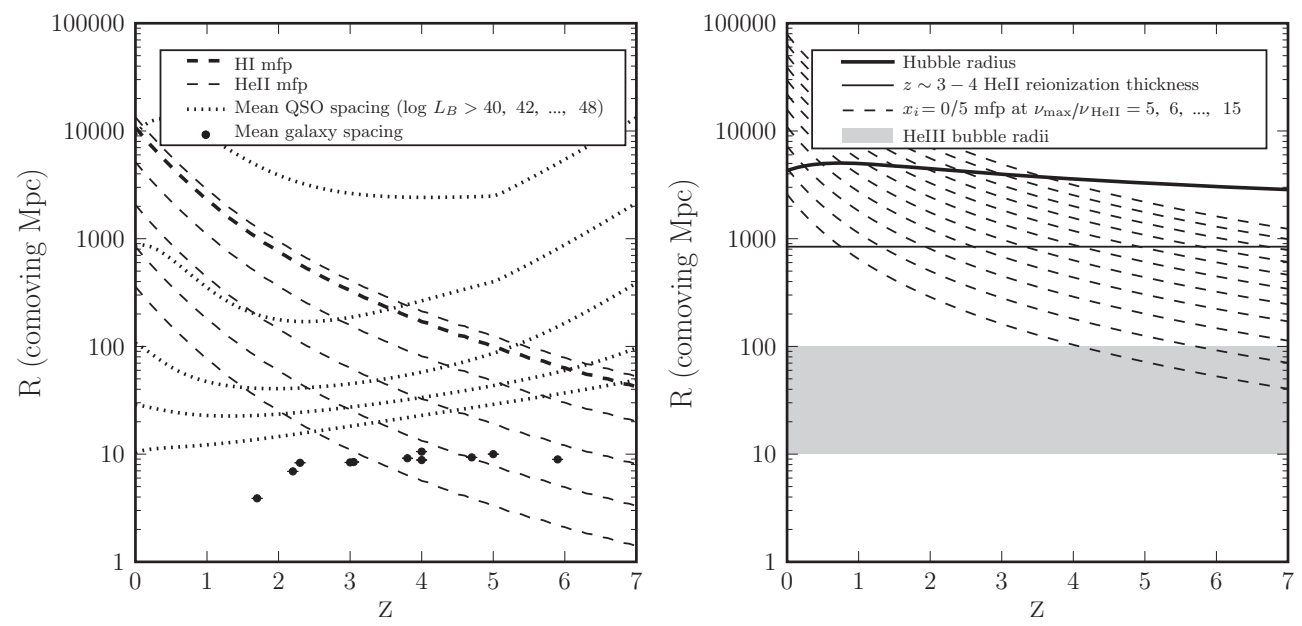

Figure 9. Important physical scales for cosmological radiative transfer. The left panel compares the mean free paths of 1 Ryd (H I ionizing; thick dashed) and 4 Ryd (He II ionizing; thin dashed) photons to the mean separation between the sources of the ionizing background. The different He II ionizing mean free path curves correspond to different $\Gamma_{\mathrm{He} \mathrm{II}}$ assumed in the calculation and ignore He II reionization. From top down, $\Gamma_{\mathrm{He} \mathrm{II}}=10^{-13}, 10^{-14}, 10^{-15}, 10^{-16}$, and $10^{-17} \mathrm{~s}^{-1}$, assuming a constant $\Gamma_{\mathrm{HI}}=0.5 \times 10^{-12} \mathrm{~s}^{-1}$. The mean separation between $L^{\star}$ galaxies vs. redshift is shown by the black points and calculated from measured galaxy UV luminosity functions (Steidel et al. 1999; Sawicki \& Thompson 2006; Yoshida et al. 2006; Bouwens et al. 2007; Reddy et al. 2008). For the mean spacing between quasars, the dotted curves correspond to different lower $B$-band luminosity cuts and are calculated using the Hopkins et al. (2007) luminosity function. From bottom up, $L_{B} \geqslant 10^{40}, 10^{42}, 10^{44}, 10^{46}$, and $10^{48} \mathrm{erg} \mathrm{s}^{-1}$. The right panel shows scales relevant to understanding the possible effects of He II reionization by quasars at $z \sim 3-4$. The comoving Hubble radius $c(1+z) / H(z)$ is indicated by the thick solid curve and the comoving distance between $z=3$ and $z=4$ spatial surfaces, labeled the "thickness of He II reionization," is shown by the thinner solid curve. The dashed curves show the mean free path of high-energy He II ionizing photons versus redshift assuming a constant ionized fraction $x_{i}=0.5$ and that the He II is uniform distributed, $\Delta l_{\mathrm{mfp}}(z) \equiv\left[\sigma_{\mathrm{He} \mathrm{II}}(v) n_{\mathrm{He} \mathrm{II}}(z)\right]^{-1}$. The curves, from bottom up, correspond to individual frequencies $v_{\max }=(5,6, \ldots, 15) v_{\mathrm{He} \text { II }}$ (see 7.3.1 for the significance of $v_{\max }$ ). The gray-shaded area indicates typical He III ionized bubble radii during He II reionization.

the ionizing background during He II reionization can be gained by considering idealized cases. We consider two such cases: (1) a single quasar at the center of an isolated ionized bubble and (2) a point in a large He II patch that has yet to be reionized.

Key insight into the ionizing background is gained by considering relevant physical scales. The left panel of Figure 9 compares the mean free paths (calculated as in Appendix D) of $1 \mathrm{Ryd}$ ( $\mathrm{H}$ I ionizing) and $4 \mathrm{Ryd}$ (He II ionizing) photons to the mean separation between the sources of the ionizing background, while the right panel shows scales relevant to understanding the possible effects of $\mathrm{He}$ II reionization by quasars at $z \sim 3-4$. The He II ionizing mean free paths are calculated by converting the H I column densities to He II assuming a constant $\Gamma_{\mathrm{H}_{\mathrm{I}}}=0.5 \times 10^{-12} \mathrm{~s}^{-1}$ and varying $\Gamma_{\mathrm{He} \text { II }}$. Since emission from star-forming galaxies provides most of the hydrogen photoionization rate at $z \gtrsim 3$ (Section 5) and the mean separation between $L^{\star}$ galaxies is much smaller than the $\mathrm{H}$ I ionizing mean free path at all redshifts $z \lesssim 6$ considered, ${ }^{5}$ it is a good approximation to treat the stellar emissivity as a uniform volume average as in Equation (2). It is also similarly the case for $\mathrm{H} \mathrm{I}$ ionizing quasar emissivity at redshifts $z \lesssim 3$, where quasars are relatively abundant and the $\mathrm{H}_{\mathrm{I}}$ ionizing mean free path large, though with larger fluctuations expected from the smaller number of quasars within each mean free path (for more detailed studies of UV background fluctuations, see Zuo 1992b, 1992a; Fardal \& Shull 1993; Croft et al. 1999, 2002; Gnedin \& Hamilton 2002; Meiksin \& White 2003, 2004; Croft 2004). Thus, it is a reasonable approximation at all redshifts to calculate the ionizing background between 1 and 4 Ryd using a volume average emissivity.

\footnotetext{
5 At redshifts $z \gtrsim 4$, the estimated mean free path relies on an extrapolation of the measured column density distribution and so the conclusion should accordingly be treated with caution. In particular, the conclusion is likely to
} break down if $\mathrm{HI}$ reionization ends at $z \approx 6$ (e.g., Fan et al. 2002, 2006b).
The situation is however quite different beyond $4 \mathrm{Ryd}$, where continuum opacity owing to He II dominates. In fact, the mean free path of He II ionizing photons at these energies, which depends on the local He II photoionization rate, is generally smaller than the mean free path of 1 Ryd H I ionizing photons, since even quasars produce relatively few photons above $4 \mathrm{Ryd}$. For example, near the peak of the quasar luminosity function at $z=2.1, S=\Gamma_{\mathrm{H}_{\mathrm{I}}} / \Gamma_{\mathrm{He} \text { II }}=140$ (Bolton et al. 2006). The relative rarity of quasars and shortness of the He II ionizing mean free path combine to create a situation in which often a single bright quasar contributes to the local He II ionizing flux. This is the case even after He II reionization has completed and results in substantial fluctuations in the ionizing background above 4 Ryd. These fluctuations could be important for metal absorption line studies and will be addressed in future work (for recent studies of the He II ionizing background fluctuations, see Bolton et al. 2006; Furlanetto 2008, 2009). Prior to the complete reionization of He II, ionized bubble walls will further limit the exposure of a given point to the He II ionizing fields of distant quasars. The radii of He III bubbles depend, at least until they percolate, on the ionizing luminosity of the central quasars and the duration for which these have been shining. This gives rise to a wide range of scales, depending on the specific quasar model, but by the middle of He II reionization (determined by an ionized fraction $\left.x_{i} \sim 0.5\right)$ the bubble radii $R_{b} \sim 10-100$ comoving $\mathrm{Mpc}$ (corresponding to a few to 20-25 proper Mpc at $z=3-4$ ) are representative (e.g., Furlanetto \& Oh 2008; McQuinn et al. 2009). A volume average uniform emissivity is then clearly inappropriate.

\subsubsection{Quasar Within an Isolated Ionized Bubble}

Consider first a point within an isolated He III bubble occupied by a single quasar at the center, $r=0$. Locally neglecting cosmological effects, the specific intensity of a radial ray is 

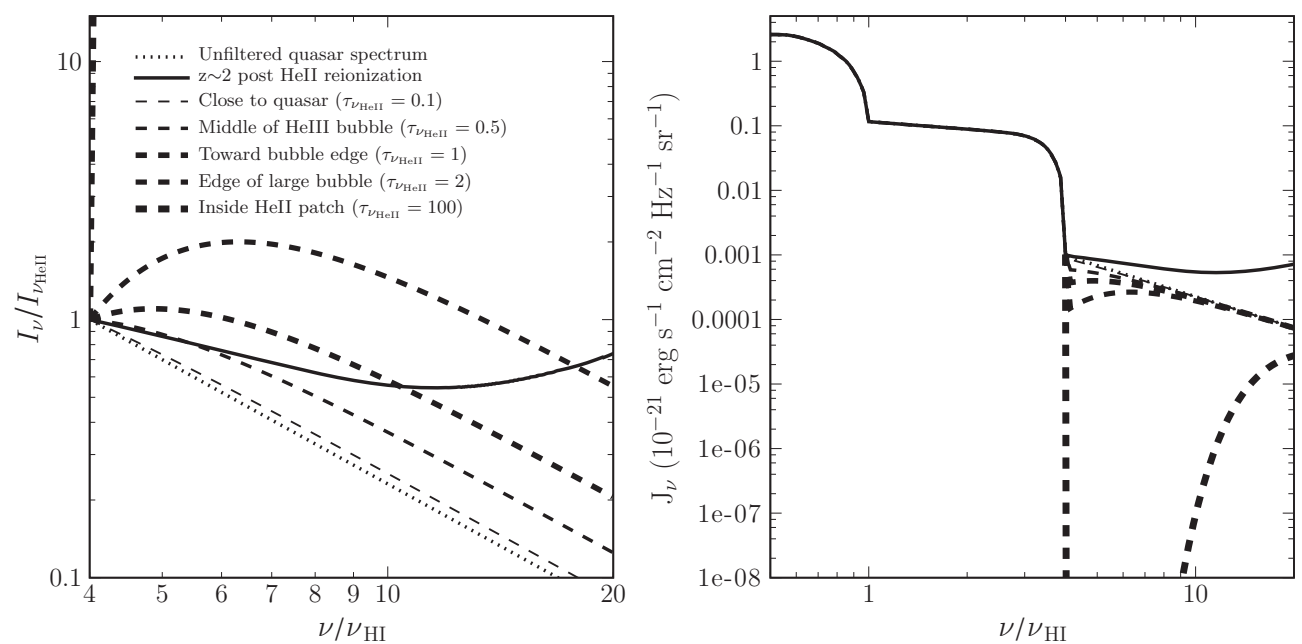

Figure 10. Left: normalized spectral hardening above the He II ionization edge. The unhardened quasar spectrum (dotted curve) is assumed to be a power law $I_{v} \propto v^{-1.6}$ (e.g., Telfer et al. 2002). The solid $z \sim 2$ post He II reionization curve shows the limit in which the mean free path is sufficiently large to contain several quasar sources, with the HI column density distribution and softness parameter $S=\Gamma_{\mathrm{HI}} / \Gamma_{\mathrm{He}} \mathrm{II}=140$ measured at $z \approx 2$ (Bolton et al. 2006). This limit is representative of the hardening in the calculations of Section 5 . The dashed curves show the hardened spectrum at different optical depths from a central quasar at the center of an isolated He III bubble. From the thinnest to the thickest curve, $\tau_{v_{\mathrm{He}} \text { II }}=0.1,0.5,1,2$, and 100 . The curves are pictorially labeled assuming a He II ionizing mean free path comparable to the bubble size, from close to the quasar to toward the edge of a large bubble and inside a He II patch. Right: the illustration of the absolute effect of He II attenuation on the full background spectrum. In addition to the spectral hardening, the spectrum is exponentially suppressed above the He II ionization edge. Recombination re-emission is omitted here but discussed in Section 7.2.

given by

$$
I_{\nu}=I_{\nu}(r=0) e^{-\tau_{\nu}(r)} .
$$

In addition to the intensity being exponentially suppressed, the spectral shape is altered by the frequency dependence of the optical depth

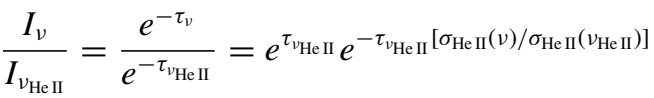

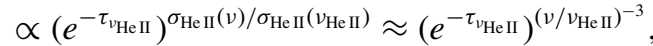

where the last equality holds approximately just above the He II ionization edge, and we have neglected the fractionally small $\mathrm{H} \mathrm{I}$ continuum opacity. It follows that the magnitude of the specific intensity is set by the optical depth at the He II ionization edge to the source, with the spectral shape entirely determined by the frequency dependence of the photoionization cross section at a given optical depth, in addition to the intrinsic spectrum of the source.

In the left panel of Figure 10, we show that a quasar spectrum $I_{v}(r=0) \propto v^{-1.6}$ is hardened as a function of $\tau_{\nu_{\text {Не II }}}$, the optical depth at the He II ionization edge from the source. The curves are pictorially labeled assuming a He II ionizing mean free path comparable to the He III bubble size, so that $\tau_{v_{\mathrm{HeII}}} \sim 1$ is near the edge of an isolated bubble centered on the quasar. We also show a $z \sim 2$ post He II reionization case in which the mean free path is sufficiently large to contain several quasar sources, with the $\mathrm{H}$ I column density distribution and softness parameter $S=\Gamma_{\mathrm{H}_{\mathrm{I}}} / \Gamma_{\mathrm{He} \text { II }}=140$ measured at $z \approx 2$ (Bolton et al. 2006). This limit is representative of the hardening in the calculations of Section 5. Note that as $\tau_{\nu_{\text {НеI II }}} \rightarrow \infty$, the spectral shape can be arbitrarily hardened just above the He II ionization edge. As $v \rightarrow \infty$ and $\sigma_{\mathrm{He} \mathrm{II}}(v) \rightarrow 0$, the spectrum returns to the unfiltered case.

The rarity of quasars implies that around an individual object the specific intensity obeys Equation (12), in which a single source is attenuated with distance, rather than a solution involving a volume average emissivity as in Equation (2). Why, though, does the ordinary optical depth $\tau_{v}$ enter in Equation (12) instead of the effective optical depth $\bar{\tau}$ as in Equation (2)? Any given light ray is always attenuated according to the intervening ordinary optical depth $\tau_{\nu}$. However, the optical depth between two points separated by a fixed distance (at fixed frequency and redshifts) fluctuates depending on their particular spatial positions because of the stochastic nature of the intervening absorbers. The effective optical depth captures the average attenuation through $e^{-\bar{\tau}}=\left\langle e^{-\tau}\right\rangle$. It is an appropriate quantity for the ionizing background between 1 and 4 Ryd, where the local intensity is an average over the light received from sources in all directions within one mean free path. The radiation above 4 Ryd at a given point in the vicinity of a quasar prior to and during He II reionization will often be dominated by the local quasar and therefore be uniquely attenuated as in Equation (12).

\subsubsection{Point in a Large He II Patch}

A point within a He II patch that has not yet been reionized ${ }^{6}$ will see a similarly hardened spectrum, but with a much stronger suppression at the He II ionization edge owing to the large intervening optical depth. The optical depth at the He II ionization edge, as a function of redshift and path length $L$, in a medium in which all the helium is assumed to be in the form of $\mathrm{He}$ II is given by

$$
\begin{aligned}
\tau_{\nu_{\text {Hel II }}^{\text {neutral }}}= & \sigma_{\text {He II }}\left(\nu_{\text {He II }}\right) n_{\mathrm{He} \mathrm{II}}(z) L=318\left(\frac{1+z}{4.5}\right)^{4} \\
& \times\left(\frac{L}{10 \text { comoving Mpc }}\right) .
\end{aligned}
$$

In He II patch, the intensity of the background at the ionization edge is therefore expected be almost entirely suppressed. As

\footnotetext{
6 In reality, the He II ionization fronts are quite smooth and extended, since they are driven by a hard spectrum (e.g., McQuinn et al. 2009). Except at the very beginning, few points have been truly untouched by He II reionization, but the discussion holds wherever the ionized fraction has not exceeded, say, $\sim 1 / 2$.
} 
$v \rightarrow \infty$ and $\sigma_{\text {He II }}(v) \rightarrow 0$, however, the optical depth drops quickly and the intensity of the background recovers. The corresponding increase of the mean free path with energy leads to the presence of a spatially smooth high-energy radiation background permeating most of the cosmic volume, as seen for example in the numerical simulations of McQuinn et al. (2009). The right panel of Figure 10 shows how the fiducial spectrum of the ionizing background at $z=3.5$, as calculated in Section 5, is altered shortward of the He II ionization edge as a function of the intervening optical depth. Note, in particular, the tremendous He II edge suppression even in the moderate case of $\tau_{\nu_{\text {НеII }}}=100$.

\subsection{Recombination Lines During He II Reionization}

The photoionization rate and ionization state of hydrogen are unaffected by the presence of He II before and during He II reionization apart from a small contribution by photons above 4 Ryd. Consequently, only the He II recombination processes are significantly modified. Of these, the most important is He II Ly $\alpha$ which imprints a distinctive line feature at 3 Ryd (Figure 4); $\mathrm{He}$ II LyC and BalC only slightly smooth the spectrum at the $\mathrm{He}$ II and $\mathrm{H}$ I ionization edges, respectively, and contribute only marginally to the photoionization rates (Figures 2 and 5).

Equations (B7) and (B8) compactly capture the behavior to He II Ly $\alpha$ re-emission. As explained in the previous section, before He II reionization begins the background spectrum is almost completely suppressed above 4 Ryd by the large optical depth at these energies. Since He II Ly $\alpha$ re-emission scales with the He II ionizing spectrum (with saturation in the optically thick limit), it will be absent before the start of He II reionization. Similarly, no He II Ly $\alpha$ should arise within He II patches during He II reionization. However, He II Ly $\alpha$ will be re-emitted within ionized bubbles illuminated by the local quasars. As He II reionization proceeds, the distance between neighboring bubbles should quickly become smaller than the mean free path for the LyC absorption of 3 Ryd photons by H I (at 1 Ryd, Figure 9 shows the mean free path to be about 200 comoving $\mathrm{Mpc}$ at $z=3.5$; at $3 \mathrm{Ryd}$, Equation (D3) predicts the mean free path to be longer by a factor of $3^{3(\beta-1)} \approx 5$ ) that governs the attenuation of He II Ly $\alpha$ radiation. In the regime in which this mean free path contains several ionized bubbles, the volume fraction of reionized He II can be viewed as the fraction of the IGM reemitting in He II Ly $\alpha$ and the He II Ly $\alpha$ re-emission line of the background spectrum can be expected to be about this fraction times the fully reionized value.

\subsection{Heat Input During He II Reionization}

The photons that ionize He II atoms in general carry more energy than the $h v_{\mathrm{He}}$ required. The residual energy is converted into kinetic energy of the resulting free electron and He III nucleus, with the frequent Coulomb collisions leading to rapid thermalization. This process of photoheating is at work at all times and for all species present. Its effect is however much more important during reionization, when atoms are being ionized at a much greater rate. The effects of He II reionization on the thermal state of the gas in cosmological simulations has so far generally be modeled by artificially boosting the photoheating rate calculated from a prescribed spatially homogeneous background instantaneously (e.g., Bryan \& Machacek 2000; Theuns et al. 2002; Jena et al. 2005), or ignoring it altogether. This approach, of limited physical basis, is a serious limitation of these simulations given the growing body of evidence that He II reionization occurs at observable redshifts $z \sim 3-4$ and is certain to manifest itself to some extent.

While cosmological radiative transfer simulations are beginning to self-consistently treat gas thermodynamics during He II reionization (e.g., Paschos et al. 2007; McQuinn et al. 2009), it is likely that the vast majority of simulations performed in the near to moderate future will not explicitly incorporate radiative transfer, either due to the computational cost or to the unavailability of an appropriate code. It therefore remains important to develop ways of approximately treating the effects of He II reionization in those simulations. We examine this problem in this section. Specifically, we consider the questions: How much does He II reionization heat the IGM? Over what timescale? And how can we approximately model its effects in standard cosmological $N$-body and hydrodynamical codes such as GADGET (Springel et al. 2001; Springel 2005), Hydra (Pearce \& Couchman 1997), or Enzo (O'Shea et al. 2004)?

The simple analytic models that follow are motivated by and owe much to the physical picture of He II reionization suggested by the radiative transfer calculations of McQuinn et al. (2009). We refer to that work for many original insights.

\subsubsection{Heat Input Calculation}

In order to gain physical intuition, we begin with a simplified model. Suppose that all the photons up to frequency $v_{\max }$ emitted by a population of sources with intrinsic spectral index $\alpha_{\mathrm{UV}}$ are absorbed by He II atoms. Then the mean energy injected into the IGM per ionization is given by

$$
\begin{aligned}
\left\langle E_{i}\right\rangle & =\frac{\int_{v_{\mathrm{HeI}}}^{v_{\max }} d v /(h v)\left(h v-h v_{\mathrm{He}}\right) v^{-\alpha_{\mathrm{UV}}}}{\int_{v_{\mathrm{HeII}}}^{v_{\max }} d v /(h v) v^{-\alpha_{\mathrm{UV}}}} \\
& =h v_{\mathrm{He} \text { II }}\left[\frac{\alpha_{\mathrm{UV}}}{\alpha_{\mathrm{UV}}-1} \frac{\left(1-x^{\alpha_{\mathrm{UV}}-1}\right)}{\left(1-x^{\alpha_{\mathrm{UV}}}\right)}-1\right] \\
& \approx \frac{h v_{\mathrm{He} \text { II }}}{\alpha_{\mathrm{UV}}-1}\left(1-\alpha_{\mathrm{UV}} x^{\alpha_{\mathrm{UV}}-1}\right),
\end{aligned}
$$

where $x \equiv v_{\text {He II }} / v_{\max }$, and the last equality holds approximation for $x \ll 1$ and $\alpha_{\mathrm{UV}}>0$. This equation neglects redshifting of the photons before absorption, which is a reasonable approximation if He II reionization lasts $\Delta z \approx 1$ at $z \sim 3-4$ (e.g., Furlanetto \& Oh 2008; Faucher-Giguère et al. 2008b; McQuinn et al. 2009). Here, the effects of spectral filtering (e.g., Abel \& Haehnelt 1999; Bolton et al. 2004; Tittley \& Meiksin 2007; Bolton et al. 2009) are incorporated in the prescribed frequency cutoff $v_{\max }$.

If all the helium is initially in the form of He II and hydrogen is fully ionized, the temperature increase is obtained by distributing the injected energy over all particles. After thermal equilibrium has been reached

$$
\begin{aligned}
\Delta T_{\mathrm{He} \mathrm{II}} & =\frac{2}{3 k} \frac{n_{\mathrm{He}}}{n_{\mathrm{tot}}}\left\langle E_{i}\right\rangle=15550 \mathrm{~K}\left[\frac{\alpha_{\mathrm{UV}}}{\alpha_{\mathrm{UV}}-1} \frac{\left(1-x^{\alpha_{\mathrm{UV}}-1}\right)}{\left(1-x^{\alpha_{\mathrm{UV}}}\right)}-1\right] \\
& \approx 31100 \mathrm{~K}\left(\frac{0.5}{\alpha_{\mathrm{UV}}-1}\right)\left(1-\alpha_{\mathrm{UV}} x^{\alpha_{\mathrm{UV}}-1}\right) .
\end{aligned}
$$

Here, $n_{\mathrm{tot}}=2 n_{\mathrm{H}}+3 n_{\mathrm{He}}$ is the total number density of particles including free electrons. Note that the total number of particles is slightly less before He II reionization owing to the smaller number of free electrons. The fractional change of $1 / 16$ is however negligible. Although the use of a sharp frequency cutoff $v_{\max }$ is a simplification of the radiative transfer, McQuinn et al. 
(2009) show that a simple argument like this one gives a good estimate of the heat input determined from detailed radiative transfer simulations.

What is the relevant value of $v_{\max }$ ? A reasonable guess is the value such that the mean free path of photons of this frequency equals the "thickness" of He II reionization. Photons of higher frequency (and therefore longer mean free path) will typically not be absorbed before He II reionization is complete. The right panel of Figure 9 shows where the mean free path intersects the thickness of He II reionization, assuming that the bulk of the latter takes place between $z=3$ and $z=4$, for different values of $v_{\max }$. For this purpose, we calculate the mean free path $R_{\mathrm{mfp}}\left(v_{\max }\right)=\left[n_{\mathrm{He}} \sigma_{\mathrm{He} \text { II }}\left(v_{\max }\right)\right]^{-1}$ assuming homogeneously distributed $50 \%$ ionized $\mathrm{He}$ II at $z=3.5$. Under these conditions (accounting for some uncertainty on the thickness of He II reionization) we expect $x^{-1} \sim 8-12$. Figure 11 shows the corresponding heat input owing to He II reionization for different value of the spectral index $\alpha_{\mathrm{UV}}$. For spectral indexes $\alpha_{\mathrm{UV}} \sim 1.5$ (Section 5.1), the heat input depends only weakly on our rough estimate of $x^{-1}$

Having obtained simple estimates for the total heat input during He II reionization, we proceed to make the derivation more rigorous, which also allows us to trace the time evolution of the heat injection. Specifically, we replace the sharp frequency cutoff by a calculation taking into account the fraction of photons emitted at each frequency at any given redshift that is absorbed during He II reionization:

$\left\langle E_{i}\right\rangle(z)=\frac{\int_{z}^{\infty} d t \int_{v_{\mathrm{He} I I}^{\prime}}^{\infty} d v^{\prime} /\left(h v^{\prime}\right)\left(h v^{\prime}-h v_{\mathrm{He} I \mathrm{II}}^{\prime}\right) \epsilon_{v^{\prime}}^{\mathrm{QSO}, \mathrm{com}}\left(z^{\prime}\right)\left[1-e^{-\tau\left(v^{\prime}, z, z^{\prime}(t)\right)}\right]}{\int_{z}^{\infty} d t \int_{v_{\mathrm{He}}^{\prime}}^{\infty} d v^{\prime} /\left(h v^{\prime}\right) \epsilon_{v^{\prime}}^{\mathrm{QSO}, \mathrm{com}}\left(z^{\prime}\right)\left[1-e^{-\tau\left(v^{\prime}, z, z^{\prime}(t)\right)}\right]}$,

where $d t=\left(d z^{\prime} / c\right)\left(d l / d z^{\prime}\right)$ and

$$
\begin{aligned}
\tau\left(v^{\prime}, z, z^{\prime}\right)= & \int_{z}^{z^{\prime}} d z^{\prime \prime} \frac{d l}{d z^{\prime \prime}} n_{\mathrm{He}}\left(z^{\prime \prime}\right)\left[1-y_{\mathrm{III}}\left(z ;{ }^{\prime \prime} \alpha_{\mathrm{UV}}\right)\right] \\
& \times \sigma_{\mathrm{He}}\left(v^{\prime \prime}=v \frac{\left(1+z^{\prime \prime}\right)}{\left(1+z^{\prime}\right)}\right)
\end{aligned}
$$

is the optical depth encountered by a photon of frequency $v^{\prime}$ emitted at redshift $z^{\prime}$ before reaching redshift $z$. Here, $n_{\mathrm{He}}$ is the proper number density of helium atoms, and a fraction $1-y_{\mathrm{III}}$ given by the reionization state is assumed to be homogeneously distributed in the form of He II, taken to be the dominant source of opacity. Equation (17) is similar to Equation (15), but with the high-frequency cutoff replaced by the smoothly varying fraction of photons absorbed $1-e^{-\tau}$ for each frequency. In addition, the mean energy injected per ionization is calculated as a function of redshift, which allows us to trace the heat input over time. While the homogeneous IGM approximation is obviously a simplification, it is a reasonable assumption for this heuristic calculation. In fact, the potential error introduced by neglecting the inhomogeneities is most important for $\tau \sim 1$. However, the strong frequency dependence of the He II photoionization cross section implies that the range of photon energy for which $\tau \sim 1$ is narrow. Moreover, for a quasar spectral index $\alpha_{\mathrm{UV}} \approx 1.5$, the cruder estimate of Figure 11 indicates that the heat input is only weakly sensitive to the exact maximum energy of the absorbed photons. These effects combine to make the uniform IGM approximation relatively robust for this particular calculation. Ultimately, though, the calculation is motivated by the fact that it reproduces the results of the full radiative transfer calculations of McQuinn et al. (2009) well.

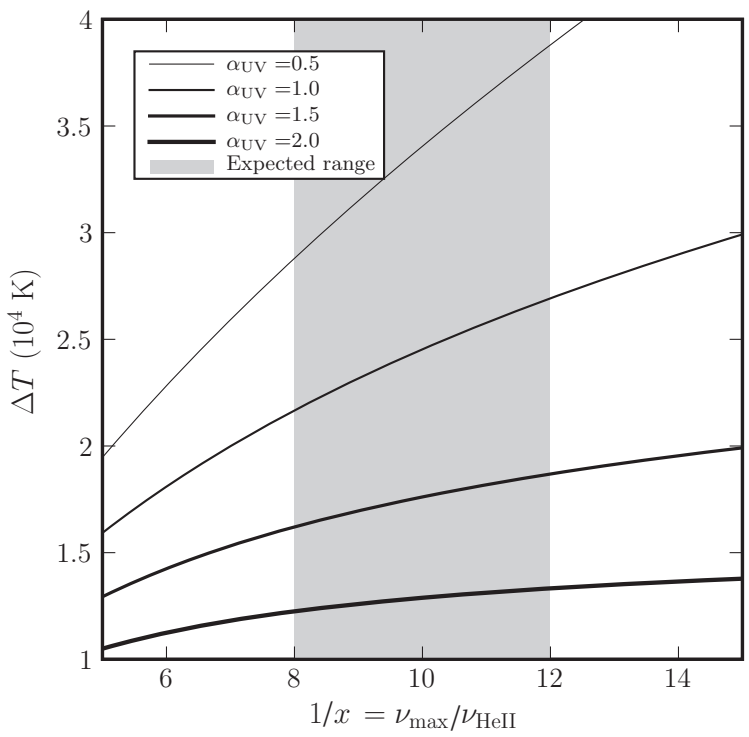

Figure 11. Total temperature increase owing to He II reionization photoheating as a function of the maximum absorbed frequency for different spectral indices $\alpha_{\mathrm{UV}}$ of the reionizing sources. The "expected range" corresponds to the bulk of He II reionization occurring between $z=3$ and $z=4$ and is shown only for suggestive purposes. Figure 12 presents more rigorous calculations that avoid fixing a hard cutoff frequency based on the He II reionization history calculated from the quasar luminosity function.

Since at a given redshift $z$, only a fraction $y_{\text {III }}$ of the He II has been reionized, the temperature increase contributed by $\mathrm{He}$ II reionization at that redshift, neglecting cooling, is given by

$$
\Delta T_{\mathrm{He} \text { II }}(z)=\frac{2}{3 k} \frac{n_{\mathrm{He}}}{n_{\mathrm{tot}}} y_{\mathrm{III}}(z)\left\langle E_{i}\right\rangle(z)
$$

The temperature of a cosmic gas parcel is in general determined by all the processes by which it gains heat and cools as it evolves, including adiabatic heating and cooling, shock heating, photoheating, Compton cooling off microwave background photons, and recombination cooling (e.g., Hui \& Gnedin 1997). Instructive intuition can however be gained from idealized solutions.

In the limit of early $\mathrm{H}_{\mathrm{I}}$ reionization (with the reionization of He I assumed to proceed simultaneously), the temperature at mean density $T_{0}$ reaches a "thermal asymptote" determined by the competition between adiabatic cooling and photoheating and whose value depends on the He II ionization state. For a powerlaw background spectrum $J_{v} \propto v^{-\alpha_{\mathrm{bg}}}$ just above the ionization edges, a good approximation to the thermal asymptote is given by

$$
\begin{aligned}
T_{0}^{\text {asymp }}(z)= & 2.49 \times 10^{4} \mathrm{~K}\left(0.464+0.536 y_{\mathrm{III}}\right)\left(2+\alpha_{\mathrm{bg}}\right)^{-1 / 1.7} \\
& \times\left(\frac{1+z}{4.9}\right)^{0.53}
\end{aligned}
$$

(Hui \& Haiman 2003). To first-order and ignoring inhomogeneities, the effect of He II reionization is to inject additional heat to each gas parcel. As the universe expands, the extra heat is diluted by adiabatic cooling, $T(z)=T\left(z^{\prime}\right)\left[(1+z) /\left(1+z^{\prime}\right)\right]^{2}$, so that an estimate of the overall temperature evolution in the early $\mathrm{H}$ I reionization limit accounting for $\mathrm{He}$ II reionization heat input is given by

$$
T(z) \approx T_{0}^{\mathrm{asymp}}(z)+\int_{\infty}^{z} d z^{\prime} \frac{d \Delta T_{\mathrm{He} \text { II }}\left(z^{\prime}\right)}{d z^{\prime}}\left(\frac{1+z}{1+z^{\prime}}\right)^{2} .
$$



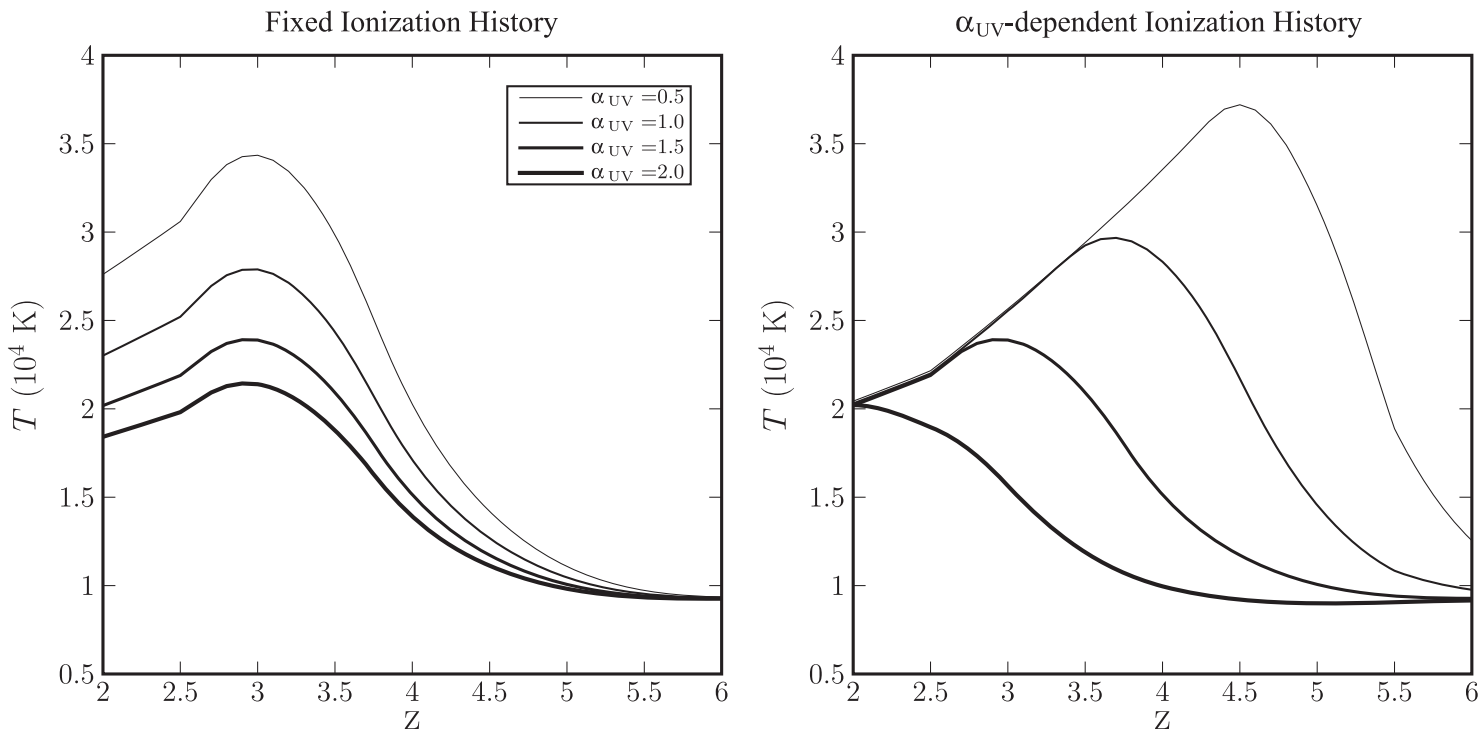

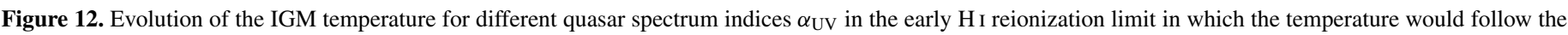

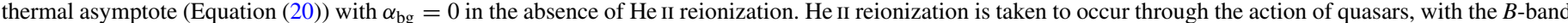

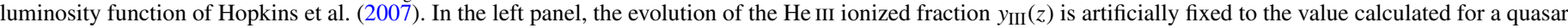

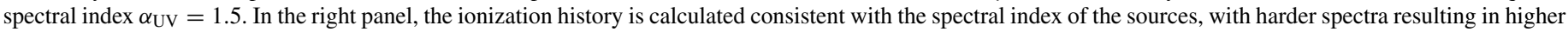
ionizing photon output rates and thus earlier reionization.

Figure 12 shows thermal histories calculated using this equation assuming a background spectral index $\alpha_{\mathrm{bg}}=0$ and the Hopkins et al. (2007) quasar luminosity function in the $B$-band for different spectral indices of the He II ionizing sources $\alpha_{\mathrm{UV}}$. Harder spectral indices are seen to result in greater heat injections, which simply owes to the larger fraction of ionizations caused by high-energy photons. Moreover, the magnitude of the total heat input as a function of spectral index is consistent with the simpler estimates using a sharp frequency cutoff shown in Figure 11. At fixed $B$-band luminosity, harder spectral indices result in higher ionizing photon output rates and thus earlier He II reionization.

The He III fraction $y_{\text {III }}$ in the above equations is obtained by counting the number of He II ionizing photons emitted by quasars as in Faucher-Giguère et al. (2008b), and we have assumed a gas clumping factor $C=5$.

\subsubsection{Scatter in the Temperature-Density Relation}

The thermal history calculations of the previous section implicitly assumed that the universe is homogeneous at a mean density and that He II reionization happens simultaneously throughout. In reality, the IGM is characterized by density fluctuations and the quasars that putatively drive He II reionization turn on at different times at different locations owing to cosmic variance. These inhomogeneities imply that the IGM temperature is not fully described by a single redshift-dependent number $T(z)$ but in reality exhibits a temperature-density relation $T(z ; \Delta)$ with some scatter about the mean at each redshift.

In the absence of He II reionization, Hui \& Gnedin (1997) showed that the temperature-density relation at $z=2-4$ is well approximated by a power law $T(z ; \Delta)=T_{0} \Delta^{\beta}$. In the limit of early $\mathrm{H}$ I reionization, $\beta \rightarrow 0.62$ as a result of the competition between photoheating and adiabatic cooling. We are interested in how this result is modified by He II reionization. Equation (21) can be generalized to

$$
T(z) \approx T_{0}^{\text {asymp }}(z) \Delta^{\beta}+\kappa \int_{\infty}^{z} d z^{\prime} \frac{d \Delta T_{\mathrm{He} \mathrm{II}}\left(z^{\prime}\right)}{d z^{\prime}}\left(\frac{1+z}{1+z^{\prime}}\right)^{2},
$$

where $\beta$ is set to the value that would be obtained without He II reionization and $\kappa$ is a stochastic factor that accounts for the fact that different regions are heated at different times by He II reionization photoheating. Our task is then reduced to determining the distribution function of $\kappa$ to estimate the scatter in the temperature-density relation.

One of the results highlighted by the radiative transfer simulations of McQuinn et al. (2009) is that much of the heating during He II reionization by quasars results from ionizations by the diffuse background of high-energy photons with large mean free paths that penetrate into He II patches before these are actually reionized by softer photons (for a different picture, see Bolton et al. 2009). In this picture, the longer a given region is exposed to the high-energy background before it is reionized, the more heat it receives; regions that are reionized last tend to be hotter. As an ansatz, again motivated by the work of McQuinn et al. (2009), we may thus posit that $\kappa \propto t_{\text {exp,eff }}$, where $t_{\text {exp,eff }}$ is an effective exposure time to the high-energy background. Note, though, that this will not be correct at the very beginning of He II reionization before the background has had time to diffuse. We denote by $z_{\mathrm{He} \text { II }}$ the redshift at which a given gas parcel is reionized in $\mathrm{He}$ II and set

$$
t_{\mathrm{exp}, \mathrm{eff}}\left(z_{\mathrm{He} \mathrm{II}}\right) \equiv \int_{\infty}^{z_{\mathrm{He} I I}} d t y_{\mathrm{III}}(z)\left(1-y_{\mathrm{III}}(z)\right)
$$

The effective exposure time is thus the age of the universe at reionization of the gas parcel, weighted by the time-dependentionized fraction, and saturating as the latter reaches order unity. The motivation for the weighting is that the heat injection is not only proportional to the raw exposure time, but also to the intensity of the high-energy background. The ionized fraction $y_{\mathrm{III}}$ counts the number of ionizing photons emitted and is therefore a tracer of this high-energy background. The $1-y_{\mathrm{III}}$ saturation factor approximates the fact that the rate of heat input also scales with $n_{\mathrm{He} \mathrm{II}}$ and is thus suppressed toward the end of reionization.

The PDF of reionization redshifts is also straightforwardly approximated from the ionized fraction evolution, since the 

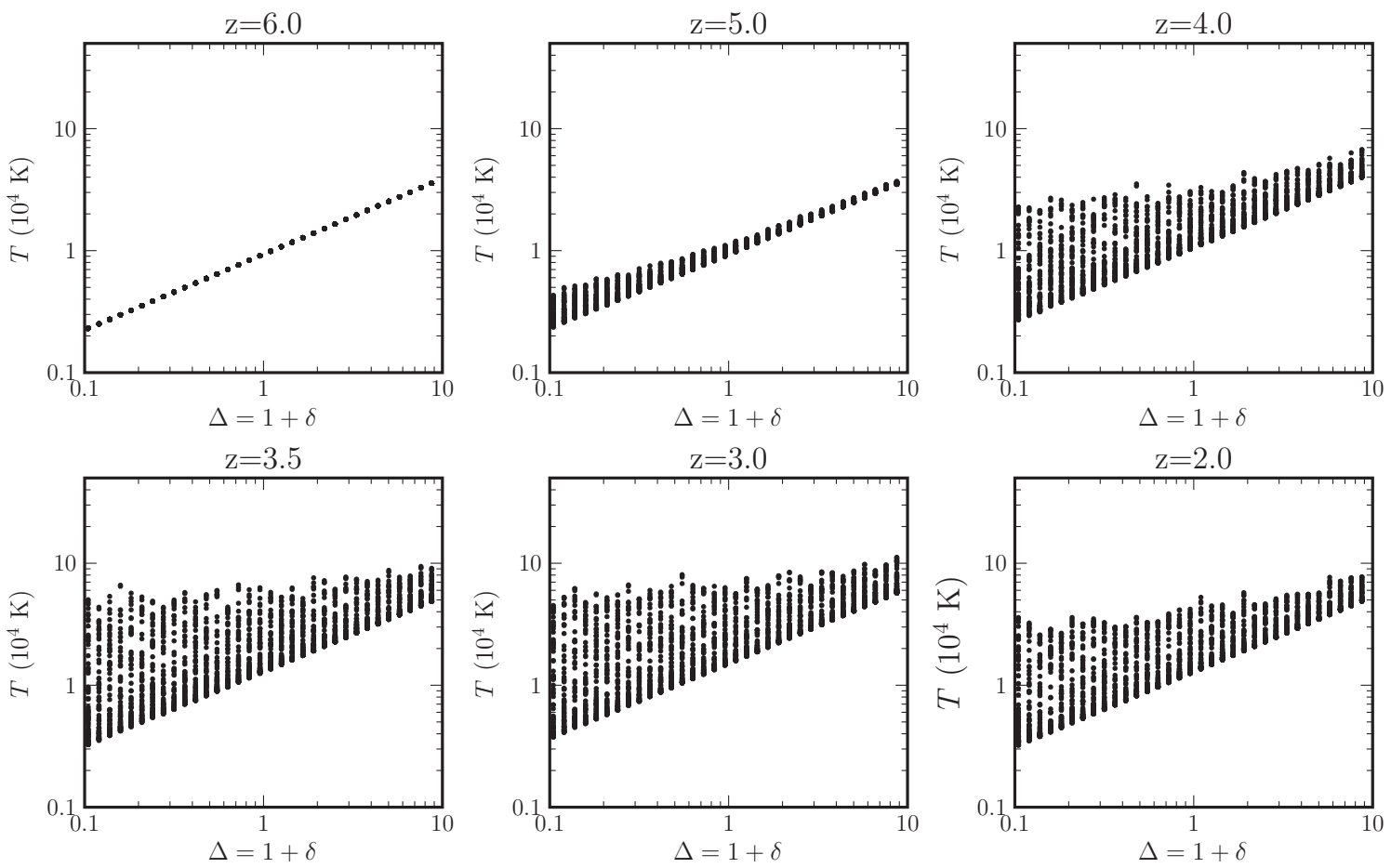

Figure 13. Evolution of the temperature-density relation in the model of Section 7.3.2 for the case of a quasar spectral index $\alpha_{\mathrm{UV}}=1.5$, with $\alpha_{\mathrm{bg}}=0$. The mean temperature at mean density traces the corresponding curve in Figure 12 by construction. The slope and scatter of the temperature-density relation are however manifest. The underlying slope is set by the early HI reionization limit, $T=T_{0} \Delta^{0.62}$, but the He II reionization heat input somewhat flattens the mean slope. The flattening arises because at low densities that are optically thin to the high-energy photons the heat deposition is density independent in the sense that gas parcels of different densities are heated by the same amount. Since the lower density elements are initially cooler, the fractional temperature increase is larger for these. He II reionization does not produce an isothermal temperature-density relation in this model because the initial heat in gas parcels above mean density is significant compared to the He II reionization heat injection and so the imprint of this initial heat is not erased.

probability of reionization during a redshift interval scales as the rate at which ionizations occur at that time:

$$
P\left(z_{\mathrm{He} \text { II }} ; z\right)= \begin{cases}y_{\mathrm{III}}\left(z_{\mathrm{He} \mathrm{II}}\right)^{-1} \frac{d y_{\mathrm{III}}}{d z}\left(z_{\mathrm{He} \text { II }}\right) & z_{\mathrm{He} \mathrm{II}} \geqslant z \\ 0 & z_{\mathrm{He} \mathrm{II}}<z .\end{cases}
$$

Energy conservation requires $\langle\kappa\rangle=1$, so we set $\kappa=$ $t_{\text {exp,eff }} /\left\langle t_{\text {exp,eff }}\right\rangle$, and in this model the scatter in the temperaturedensity relation ultimately is calculable from the quasar luminosity function. We wish to emphasize that the effective exposure time ansatz in Equation (23) was obtained heuristically and certainly does not capture the full complexity of He II reionization, though it does agree reasonably well with the results of the McQuinn et al. (2009) simulations and provides a simple way to understand them.

Figure 13 shows the evolution of the temperature-density relation in this model for the case of a quasar spectral index $\alpha_{\mathrm{UV}}=1.5$, with $\alpha_{\mathrm{bg}}=0$. The mean temperature at mean density, $\langle T(z ; \Delta=1)\rangle$, then traces the corresponding curve in Figure 12 by construction. The slope and scatter of the temperature-density relation are however manifest. The underlying slope is set by the early $\mathrm{H}_{\mathrm{I}}$ reionization limit $T=T_{0}^{0.62}$, but the He II reionization heat input somewhat flattens the mean slope. The flattening arises because at low densities that are optically thin to the high-energy photons the heat deposition is density independent in the sense that gas parcels of different densities receive the same temperature increment. Since the lower density elements are initially cooler, the fractional temperature increase is larger for these. In agreement with the simulations of McQuinn et al. (2009), He II reionization does not produce an isothermal $(\beta=0)$ temperature-density relation. This is, similarly, simply because the initial heat in gas parcels above mean density $(\Delta>1)$ is significant compared to the He II reionization heat injection and so the imprint of this initial heat is not erased. Finally, it is worth re-emphasizing that this model predicts a large scatter in the temperature-density relation, which may have important consequence for interpreting $\operatorname{Ly} \alpha$ forest data. In this model, though, the scatter arises from the scatter in the He II reionization times for different gas parcels. Locally, neighboring points will be reionized at similar times and we therefore expect the scatter in the temperature-density relation to be significantly reduced.

\subsubsection{Implementation in Hydrodynamical Codes}

Cosmological hydrodynamical simulations usually incorporate the effects of a prescribed UV background on the thermal history of the gas under the assumption of an optically thin plasma (for the relevant equations, see Katz et al. 1996a). This assumption manifestly breaks down during He II reionization and has led simulators to artificially increase photoheating rates as a rough approximation of the radiative transfer effects. A more physically motivated approach is to increase the temperature of each gas element by an amount $\left(d \Delta T_{\mathrm{He}}(z) / d z\right) \Delta z$ (which is subsequently allowed to cool) at each time step $\Delta z$ in the simulation, where $\Delta T_{\mathrm{He} \mathrm{II}}(z)$ is pre-computed given the desired He II reionization history as in Equation (19). This approach misses the scatter and inhomogeneity of the temperature-density relation discussed in the previous section but has the advantage of only requiring an additional term in the temperature equation and adding negligible computational overhead while capturing the timescale and magnitude of the heat input more realistically. It could conceivably be extended to account for spatial 
Table 2

Photoionization and Photoheating Rates for Our Fiducial Model

\begin{tabular}{|c|c|c|c|c|c|c|c|}
\hline$z$ & $\begin{array}{c}\Gamma_{\mathrm{HI}}^{\mathrm{a}} \\
10^{-12} \mathrm{~s}^{-1}\end{array}$ & $\begin{array}{c}\Gamma_{\mathrm{He} \mathrm{I}}{ }^{\mathrm{a}} \\
10^{-12} \mathrm{~s}^{-1}\end{array}$ & $\begin{array}{c}\Gamma_{\text {He II }}{ }^{a} \\
10^{-12} \mathrm{~s}^{-1}\end{array}$ & $\begin{array}{c}\dot{q}_{\mathrm{HI}}{ }^{\mathrm{a}} \\
10^{-12} \mathrm{eV} \mathrm{s}^{-1}\end{array}$ & $\begin{array}{c}\dot{q}_{\mathrm{He} \mathrm{I}}{ }^{\mathrm{a}} \\
10^{-12} \mathrm{eV} \mathrm{s}^{-1}\end{array}$ & $\begin{array}{c}\dot{q}_{\mathrm{He} \mathrm{II}}{ }^{\mathrm{a}} \\
10^{-12} \mathrm{eV} \mathrm{s}^{-1}\end{array}$ & $\begin{array}{c}\Delta T_{\mathrm{He} \mathrm{II}}{ }^{\mathrm{b}} \\
T(\mathrm{~K}) \\
\end{array}$ \\
\hline 0.0 & 0.0384 & 0.0213 & $1.231 \times 10^{-4}$ & 0.158 & 0.141 & 0.0032 & 14269 \\
\hline 0.5 & 0.1295 & 0.0860 & $6.845 \times 10^{-4}$ & 0.569 & 0.600 & 0.0156 & 14269 \\
\hline 0.75 & 0.2082 & 0.1471 & $1.361 \times 10^{-3}$ & 0.929 & 1.064 & 0.0292 & 14269 \\
\hline 1.5 & 0.4975 & 0.3843 & $4.288 \times 10^{-3}$ & 2.260 & 3.037 & 0.0837 & 14269 \\
\hline 1.75 & 0.5630 & 0.4446 & $4.744 \times 10^{-3}$ & 2.574 & 3.579 & 0.0920 & 14269 \\
\hline 2.0 & 0.6013 & 0.4856 & $4.811 \times 10^{-3}$ & 2.768 & 3.974 & 0.0926 & 14269 \\
\hline 2.25 & 0.6142 & 0.5076 & $4.511 \times 10^{-3}$ & 2.852 & 4.196 & 0.0867 & 14269 \\
\hline 2.5 & 0.6053 & 0.5132 & $3.939 \times 10^{-3}$ & 2.839 & 4.274 & 0.0757 & 14269 \\
\hline 3.5 & 0.4849 & 0.4617 & $1.245 \times 10^{-3}$ & 2.384 & 3.933 & 0.0243 & 8007 \\
\hline 3.75 & 0.4560 & 0.4469 & $7.907 \times 10^{-4}$ & 2.272 & 3.830 & 0.0159 & 5519 \\
\hline 4.0 & 0.4320 & 0.4329 & $4.818 \times 10^{-4}$ & 2.171 & 3.737 & $9.862 \times 10^{-3}$ & 3802 \\
\hline 4.25 & 0.4105 & 0.4203 & $2.618 \times 10^{-4}$ & 2.083 & 3.657 & $5.639 \times 10^{-3}$ & 2619 \\
\hline 4.5 & 0.3917 & 0.4080 & $1.351 \times 10^{-4}$ & 2.002 & 3.580 & $3.133 \times 10^{-3}$ & 1770 \\
\hline 4.75 & 0.3743 & 0.3948 & $7.271 \times 10^{-5}$ & 1.921 & 3.493 & $1.624 \times 10^{-3}$ & 1170 \\
\hline 5.0 & 0.3555 & 0.3800 & $3.724 \times 10^{-5}$ & 1.833 & 3.393 & $8.087 \times 10^{-4}$ & 713 \\
\hline 5.25 & 0.3362 & 0.3647 & $2.060 \times 10^{-5}$ & 1.745 & 3.284 & $3.654 \times 10^{-4}$ & 394 \\
\hline 5.5 & 0.3169 & 0.3494 & $8.924 \times 10^{-6}$ & 1.661 & 3.169 & $1.448 \times 10^{-4}$ & 210 \\
\hline 5.75 & 0.3001 & 0.3327 & $5.992 \times 10^{-6}$ & 1.573 & 3.042 & $6.795 \times 10^{-5}$ & 112 \\
\hline
\end{tabular}

Notes.

a Optically thin rates.

b Cumulative temperature increase owing to He II reionization, which should be incrementally added to gas elements and then allowed to cool to model the heat input (Section 7.3.3).

inhomogeneities and the scatter in reionization times similarly to semi-analytic models being applied to efficiently model $\mathrm{H} \mathrm{I}$ reionization (e.g., Zahn et al. 2007). An alternative approach would be to replace the optically thin photoionization and heating rates by "effective" values that can be substituted into the usual optically thin equations to yield the desired result.

Table 2 tabulates both the optically thin photoionization and photoheating rates, and the $\Delta T_{\mathrm{He} \mathrm{II}}$ values to use to model the effects of He II reionization under our prescription for the quasar model with $\alpha_{\mathrm{QSO}}=1.6$ employed in the fiducial background spectrum calculations of Section 5. In general, the photoheating rate for species $i$ is given by

$$
\dot{q}_{i}=\frac{1}{4 \pi} \int_{v_{i}}^{\infty} \frac{d v}{h v} J_{v} \sigma_{i}(v)\left(h v-h v_{i}\right) .
$$

The corresponding ionized fraction versus redshift is shown by the $C=5$ curve in Figure 7 of Faucher-Giguère et al. (2008b). In this model, He II reionization completes by $z=3$, with $\sim 80 \%$ of the ionization occurring between $z=3$ and $z=4$.

\section{COMPARISON WITH PREVIOUS WORK}

As one of the motivations for our calculation of the ionizing background spectrum was to provide an alternative to the widely used models of Haardt \& Madau (1996; see also Haardt \& Madau 2001) and their informally released deriva- tives, it is useful to directly compare our results with these authors.

Before we do so, we wish to emphasize that in this work we attempted to improve on technical aspects of the calculation. In particular, we performed more self-consistent calculations of the ionization structure of individual absorbers (Section 3) and our treatment of recombination emission (Section 4) was based on approximating the results of these photoionization calculations rather than on an escape probability formalism. Moreover, all the numerical calculations presented here were performed using an independently developed code and our empirical constraints (Section 5) were also obtained independently in our previous work. The comparison of the final results against those of Haardt \& Madau thus provides an indication of the uncertainty in the resulting spectrum.

In Figure 14, we compare our fiducial model (ignoring He II reionization) with two models informally released by $\mathrm{F}$. Haardt and P. Madau in 2005 (H\&M05; F. Haardt 2005, private communication). Both models include a quasar contribution based on the Croom et al. (2004) luminosity function. One model in addition includes a stellar contribution calculated using a Bruzual \& Charlot (2003) population synthesis code assuming a Salpeter initial mass function, age $0.5 \mathrm{Gyr}$, constant star formation, and an escape fraction of $10 \%$. We compare both the detailed spectra at redshifts $z=0,3$, and 6 and the integrated photoionization rates of $\mathrm{HI}$ and $\mathrm{He}$ II versus redshift. For the 

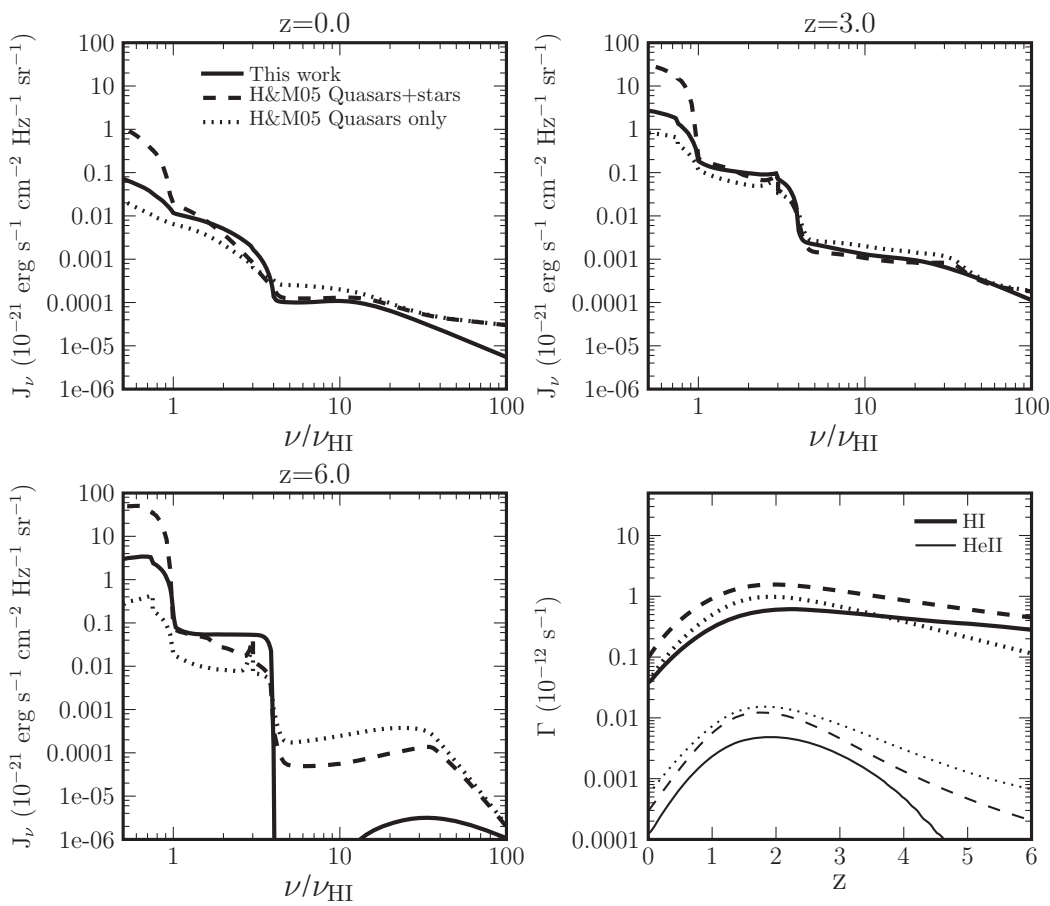

Figure 14. Comparison of our fiducial ionizing background model with models informally released by F. Haardt and P. Madau in 2005 (H\&M05). Both H\&M05 models include a quasar contribution based on the Croom et al. (2004) luminosity function. One model in addition includes a stellar contribution calculated using a Bruzual \& Charlot (2003) population synthesis code assuming a Salpeter initial mass function, age $0.5 \mathrm{Gyr}$, constant star formation, and an escape fraction of $10 \%$. First three panels: detailed spectra at redshifts $z=0,3$, and 6. For this comparison, the H\&M05 models were normalized by a factor of 0.5 to better match our fiducial calculation. Lower right: photoionization rates of $\mathrm{HI}$ and He II vs. redshift. Here, the H\&M05 models were not normalized and so the different amplitudes reflect the difference in the models as provided.

detailed spectra, the Haardt \& Madau models were normalized by a factor of 0.5 to better match our fiducial calculations in the ionizing range. As a non-negligible uncertainty remains in the amplitude of the intergalactic $\mathrm{H}$ I photoionization rate (e.g., Bolton et al. 2005; Faucher-Giguère et al. 2008b), it is fair to renormalize the models before comparing them, a procedure which is also frequently used by simulators to match the observed Ly $\alpha$ forest mean transmission (see the discussion at the end of Section 5.2). The photoionization rates shown in the last panel have however not been renormalized and so reflect the models as provided.

It is interesting that in spite of the differences in the technical treatment and the independently obtained empirical constraints, our calculations of the spectral shape in the ionizing range agree quite well with the H\&M05 models between $z=0$ and $z=3$, suggesting that the calculations are relatively robust in this redshift range. The spectra however diverge increasingly toward higher redshifts as a result of the different source prescriptions this regime. In our model, the quasar contribution drops more rapidly as $z \rightarrow \infty$, while the compensating stellar emissivity increases to maintain the nearly flat total hydrogen photoionization rate measured from the Ly $\alpha$ forest (FaucherGiguère et al. 2008a, 2008b). While the Hopkins et al. (2007) luminosity function, we use, combines different data sets to constrain the faint-end slope up to $z=4.5$, the Croom et al. (2004) luminosity function used in the H\&M05 models is based solely on the $2 \mathrm{QZ}$ survey and is only measured up to $z=2.1$. Our calculations are therefore more reliable at $z \gtrsim 3$. Since we prescribe the $\mathrm{H}$ I column density distribution, but selfconsistently calculate the He II distribution from the hardness of the background at each redshift, the decreasing $\mathrm{He}$ II to $\mathrm{H}$ I ionizing emissivity ratio results in a reduction of the He II to
H I ionizing photon mean free path, and therefore amplifies the $\Gamma_{\mathrm{HeII}} / \Gamma_{\mathrm{HI}}$ evolution. This explains the increasingly strong He II break in our model. Although He II reionization would modify the results above the He II ionization edge (Section 7.1), a prediction of our model is a larger ratio of $\mathrm{H}$ I to He II ionizing flux beyond $z \approx 3$.

A significant difference between our calculations and the original work of Haardt \& Madau (1996) is with the fraction by which recombination emission boosts the photoionization rates. For their original model, Haardt \& Madau (1996) found $\Gamma_{\mathrm{HI}}^{\text {withrec }} / \Gamma_{\mathrm{HI}}^{\mathrm{rec}}$ as high as 1.5 and $\Gamma_{\mathrm{He} \text { II }}^{\mathrm{withec}} / \Gamma_{\mathrm{He} \text { II }}^{\mathrm{rec}}$ peaking at 1.7 . For our fiducial model, we found recombinations to be less important for the photoionization rates, with $\Gamma^{\text {withrec }} / \Gamma^{\text {rec }} \lesssim 1.1$ at $z \leqslant 4$ for both $\mathrm{H} \mathrm{I}$ and He II (Figure 5; the boost factor increases somewhat toward higher redshifts as the mean free paths and leakage due to redshifting below the ionization edges decrease, as discussed in Section 6.2). The analytic model developed in Appendix $C$ helps us understand our numerical results and give us confidence in their accuracy. The differences with respect to the boost factors found by Haardt \& Madau (1996) must partly be due to the different parameters of the calculations (e.g., the column density distribution and source prescriptions), but may also originate from the different techniques used. Preliminary investigation suggests that Haardt \& Madau (1996) may have incorrectly used a case B recombination coefficient instead of the case A coefficient in a step of their escape probability calculation, resulting in an overestimate of the recombination boost which likely explains a large part of the discrepancy (F. Haardt 2009, private communication). Our results agree better with those of Fardal et al. (1998), who find that recombinations boost the photoionization rates of both $\mathrm{HI}$ and $\mathrm{He}$ II by $\sim 20 \%$. 
Recently, Madau \& Haardt (2009) proposed a new effect on the spectrum of the ionizing background which could be important particularly before He II is reionized. When a large amount of $\mathrm{He}$ II is present, the opacity arising from $\mathrm{He}$ II Ly $\beta$ and higher Lyman-series resonances produce a sawtooth absorption pattern between 3.56 and 4 Ryd and the He II Ly $\alpha$ re-emission line at $3 \mathrm{Ryd}$ is boosted by resulting degraded photons. We do not include this effect in the present work but plan to do so in the future.

\section{DISCUSSION AND CONCLUSION}

In this work, we have revisited the calculation of the UV background spectrum. The three main improvements over previous work are as follows.

1. The implementation of new empirical constraints on the sources of radiation based on a detailed study of intergalactic absorption and updated luminosity functions (FaucherGiguère et al. 2008d, 2008a, 2008b). In our favored fiducial model, star-forming galaxies play a crucial role and dominate the $\mathrm{H}$ I photoionization rate at $z \gtrsim 3$.

2. A re-examination of the radiative transfer within individual absorbers and an exploration of the physical dependences of the calculated background. In particular, we perform more self-consistent photoionization calculations including recombination emission, present a new treatment of recombination emission based on them, and clarify how the net enhancement of the photoionization rates is influenced by redshifting of the recombination photons below the ionization edges and their energy distribution.

3. A treatment of the effects of He II reionization on background spectrum and on the thermal history of the intergalactic medium.

The main argument supporting a UV background dominated by stellar emission beyond $z \approx 3$ is that while the total $\mathrm{HI}$ photoionization rate measured from the $\mathrm{Ly} \alpha$ forest is remarkably constant between at least $z=2$ and $z=4.2$ (e.g., FaucherGiguère et al. 2008a, 2008b), the quasar luminosity function is strongly peaked near $z=2$ (e.g., Hopkins et al. 2007). Thanks to large-scale Lyman break and Ly $\alpha$ line surveys (e.g., Steidel et al. 1999; Sawicki \& Thompson 2006; Yoshida et al. 2006; Bouwens et al. 2007; Reddy et al. 2008; Ouchi et al. 2008), starforming galaxies are now known to exist numerously at these redshifts and are therefore the leading candidates to account for the remaining ionizing photons. The quasar luminosity function and measurements of He II-to-H I column density ratios however indicate that quasars do contribute a large fraction of the $\mathrm{HI}$ photoionization rate at their $z \approx 2$ peak; in our fiducial model, this fraction is $2 / 3$.

The evidence in favor of a large (and dominant at the highest redshifts) contribution of star-forming galaxies to the ionizing background is supported by related and independent studies. Previous studies of the H I photoionization rate from the Ly $\alpha$ forest (Rauch et al. 1997; Haehnelt et al. 2001; Bolton et al. 2005) have in fact supported this conclusion, though with somewhat more leeway owing to larger statistical error bars. The case has also been made independently by combining direct measurements of the UV luminosity function of galaxies and of their escape fraction (e.g., Steidel et al. 2001; McDonald \& Miralda-Escudé 2001; Shapley et al. 2006; Cowie et al. 2009). Metal line studies provide a further line of evidence, indicating that a mix of stars and quasars best fits measured the abundance ratios of various ions including $\mathrm{C}$ IV, $\mathrm{S}$ IV, and $\mathrm{O}$ VI (Boksenberg et al. 2003; Schaye et al. 2003; Aguirre et al. 2004, 2008). In fact, the ionizing spectrum presented herein could be directly confronted against and further constrained by such observations. Theoretical arguments also suggest that star-forming galaxies should dominate early on (e.g., Springel \& Hernquist 2003; Hernquist \& Springel 2003; Loeb 2009).

Although the fiducial model detailed in Section 5 fits our observational constraints, it is not at present uniquely determined. As we explore in Section 6, the background depends on the details of both the sources and sinks of radiation. For instance, we have adopted a hard spectral index $\alpha_{\star}=1$ for star-forming galaxies between 1 and 4 Ryd based on the comparison of theoretical models with observational line diagnostics by Kewley et al. (2001). However, in spite of a few weak detections, the emergent spectral shape of high-redshift galaxies at these energies has yet to be directly measured owing to the large attenuation by the intervening IGM (e.g., Steidel et al. 2001; Shapley et al. 2006). Moreover, present population synthesis models are at odds with one another in their predictions in this energy range (e.g., Kewley et al. 2001; Boksenberg et al. 2003), making it difficult to rely on them with confidence. Perhaps the largest uncertainty with respect to the absorbers is the abundance and redshift evolution of Lyman limit systems, which determine the mean free path of ionizing photons. These are still poorly constrained, especially above $z \approx 4$, and introduce a commensurate uncertainty in the calculation of the amplitude of the ionizing background at these redshifts (e.g., Figure 7).

The total ionizing background receives a contribution from recombinations that re-emit other ionizing photons. We reexamined the physics of this recombination contribution. Interestingly, we find that the enhancement of the photoionization rates from recombinations is not simply a function of the number of recombination photons. This arises because many of the recombination photons rapidly redshift and leak below their corresponding ionization edges as well as from their distribution in energy. Focusing on the $\mathrm{H}$ I photoionization rate, the main factors determining $\Gamma_{\mathrm{HI}}^{\text {withrec }} / \Gamma_{\mathrm{H}_{\mathrm{I}}}^{\mathrm{rec}}$ are the parameters of the column density distribution and the $\mathrm{LyC}$ recombination line profile. Shorter ionizing photon mean free paths, relative to the recombination line width, inhibit leakage. Wider recombination lines associated with higher gas temperatures, on the other hand, produce higher energy photons that receive less weight in the photoionization rate owing to the frequency dependence of the cross section. The steepness of the column density distribution also plays a role: for steeper distributions, more of the recombinations occur in optically thin absorbers from which essentially all the recombination photons escape into the IGM, in contrast to optically thick absorbers that trap a large fraction. In general, $\Gamma_{\mathrm{HI}_{\mathrm{I}}}^{\text {withec }} / \Gamma_{\mathrm{H}_{\mathrm{I}}}^{\mathrm{rec}}$ increases toward higher redshifts as the mean free path diminishes and leakage becomes less significant. In Appendix $\mathrm{C}$, we give a simple analytical model that quantitatively captures these effects and agrees well with our full numerical calculations. We conclude that there is not a unique answer for how much recombinations boost the ionization rates, but that it depends both on redshift and on the parameters of the absorbers and their distribution.

Our main solutions to the radiative transfer problem assume that the ionizing background is homogeneous. This approximation will in particular break down during the reionization of $\mathrm{HI}$ and He II. As He II reionization may take place under the action of quasars at immediately accessible redshifts $z \sim 3-4$ (e.g., Sokasian et al. 2002; Wyithe \& Loeb 2003; Furlanetto \& Oh 2008; Faucher-Giguère et al. 2008b), we provide a discus- 
sion of its effects on both the spectrum of the ionizing background and on the thermal history of the IGM. In regions that have yet to be reionized, the spectrum is expected to be almost completely suppressed immediately above $54.4 \mathrm{eV}$ by He II absorption. However, the universe remains relatively transparent at higher energies owing the frequency dependence of the photoionization cross section. As the spectrum recovers, a background of $\gtrsim 0.5 \mathrm{keV}$ photons should thus permeate the entire universe.

Another important effect of He II reionization is to inject heat into the IGM via photoheating. We provide a simple analytical model to estimate the overall temperature increase owing to He II reionization based on energy conservation. In this model, which agrees well with the three-dimensional radiative transfer simulations of McQuinn et al. (2009), the total temperature increase depends most sensitively on the far-UV quasar spectral index. For a value $\alpha_{\mathrm{UV}}=1.5$, the temperature increase could be as much as $15,000 \mathrm{~K}$, though the effect is mitigated by simultaneous adiabatic cooling. Harder spectral energy distributions lead to more energy injection. The model is extended to understand the effects on the temperaturedensity relation under the ansatz that the local heat input scales with the effective exposure to the high-energy background. The main effects are to flatten the mean temperature-density relation with respect to the early H I reionization limit (Hui \& Gnedin 1997) and introduce a large scatter. The flattening arises because the high-energy background heat deposition per atom is independent of the local density: the temperature of initially cooler lower density regions is increased by the same amount as that of initially hotter ones of higher density. The temperature-density relation does not become fully isothermal as the He II reionization heat input is comparable to the initial thermal energy of the gas and so the memory of the latter is not erased. In our model, the scatter of the temperature-density relation originates from the scatter in the reionization times of different gas elements and can be calculated from the quasar luminosity function.

What remains to be done? As outlined above, the ionizing background spectrum is not yet uniquely determined and further direct constraints (for example, using metal line ratios) as well as constraints on its sources and sinks (which enter the theoretical calculation) are sure to continue to refine it, especially at the highest redshifts. The theoretical framework itself needs to be improved to take into account the fluctuations in the ionizing background that necessarily exist at some level and are certain to be important at least during reionization events (for work in this direction, see Bolton et al. 2006; Furlanetto $2008,2009)$. In this vein, while our idealized discussion of the effects of He II reionization provides some basic physical understanding, it leaves ample opportunity for improvement. This is a particularly exciting area for future progress as studies of He II reionization are currently blossoming, with much $\mathrm{H}$ I Ly $\alpha$ forest data already available and new He II Ly $\alpha$ forest lines of sight that the Cosmic Origins Spectrograph (Green 2000) to be installed aboard the Hubble Space Telescope is poised to deliver soon (Syphers et al. 2009), and the accompanying surge of theoretical interest. In particular, several groups are now beginning to numerically tackle the full problem of threedimensional radiative transfer and its thermal effects (Sokasian et al. 2002; Paschos et al. 2007; McQuinn et al. 2009; Maselli et al. 2009). Another interesting observational opportunity for probing the extragalactic radiation background is provided by the newly launched Fermi Gamma-Ray Space Telescope.

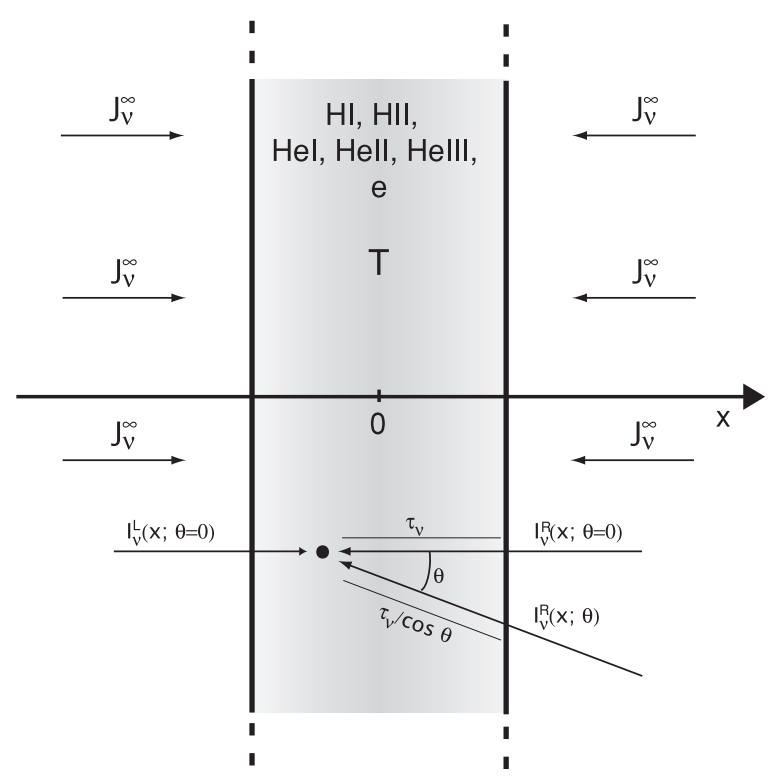

Figure 15. Definition of the geometry for our photoionization calculations. Absorbers are modeled as sheets infinite in extent, but finite in thickness. The ionization states of the ions of hydrogen and helium are calculated for a prescribed gas temperature $T$ and isotropic external radiation field of specific intensity $J_{v}^{\infty}$.

Indeed, the intervening extragalactic background light should attenuate the $\gamma$ rays from distant sources through electronpositron pair production and thus give us an additional handle on it, particularly below the $\mathrm{H}$ I ionization edge (e.g., Madau \& Phinney 1996; Oh 2001a; Razzaque et al. 2009).

To facilitate use and extension of the results presented in this work and their comparison with observations, numerical tables are available in electronic form online at http://www.cfa.harvard.edu/ cgiguere/uvbkg.html.

We thank Hy Trac for useful discussions, Francesco Haardt for comments that helped us clarify the manuscript, and the referee for a thorough and careful review. We are grateful to Matt McQuinn for physical insights in the effects of $\mathrm{He}$ II reionization on the thermal history of the IGM. C.A.F.G. is supported by a NSERC Postgraduate Fellowship and the Canadian Space Agency. This work was supported in part by NSF grants ACI 96-19019, AST 00-71019, AST 02-06299, AST 03-07690, and AST 05-06556, and NASA ATP grants NAG5-12140, NAG513292, NAG5-13381, and NNG-05GJ40G. Further support was provided by the David and Lucile Packard, the Alfred P. Sloan, and the John D. and Catherine T. MacArthur Foundations.

\section{APPENDIX A}

\section{PHOTOIONIZATION CALCULATIONS}

In this appendix, we detail the code used to calculate the photoionization equilibrium structure of individual cosmic absorbers outlined in Section 3.

We approximate the absorbers as sheets infinite in extent but finite in thickness, with geometry defined in Figure 15. Although this geometry is restrictive, the calculation is otherwise three-dimensional in the sense that it takes into account that rays incident at different angles encounter different optical depths. This geometry is clearly adequate for the sheets of the cosmic web, but somewhat inexact for the filamentary and clumpy structures, and the results may therefore be off by a correspond- 
ing geometrical factor. Nevertheless, the calculations retain the essence of the problem and significantly improve over previous work that either assumed a semi-infinite geometry and an escape probability formalism (Haardt \& Madau 1996) or gray cross sections (Fardal et al. 1998). We also for the first time self-consistently treat the coupling between hydrogen and helium arising from their recombination emission, as explained below. A more accurate approach would consider a distribution of three-dimensional absorber geometries (e.g., obtained from a cosmological simulation) for each line-of-sight optical depth considered, but would be much more involved and is beyond the scope of this work.

We assume that the slab is composed of hydrogen and helium, with cosmic mass fractions $X=0.75$ and $Y=0.25$ (e.g., Burles et al. 2001). The temperature of the gas is set to $T=2 \times 10^{4}$ $\mathrm{K}$, as estimated for optically thin $\mathrm{Ly} \alpha$ forest absorbers (McDonald et al. 2001; Zaldarriaga et al. 2001, see also Schaye et al. 2000 and Ricotti et al. 2000). While the gas temperature may differ and be more complex in structure in damped Ly $\alpha$ absorbers (DLA) with $N_{\mathrm{HI}_{\mathrm{I}}} \geqslant 2 \times 10^{20} \mathrm{~cm}^{-2}$ that are able to cool and form stars (e.g., Wolfe et al. 2005), the detailed properties of these absorbers are not crucial since absorbers with $N_{\mathrm{H}_{\mathrm{I}}} \gg$ $10^{17.2} \mathrm{~cm}^{-2}$ are completely opaque to ionizing photons regardless of their exact column density. The thickness of the slab is assumed to be equal to its Jeans length $L_{J}=\sqrt{\pi \gamma k T / G \rho \mu m_{p}}, 7$ which is both theoretically motivated and provides a good match to observations (Schaye 2001). In practice, we prescribe the physical mass density $\rho$ and derive the ionic column densities in photoionization equilibrium so that we do not need to explicitly assume a relation between $N_{\mathrm{HI}}$ and $n_{\mathrm{H}}$. Because pressure also smoothes the gas on this scale, the absorbers are assumed to be uniform in density, but we have explored other density profiles and found that our numerical results are only marginally affected, and our broad conclusions unaltered.

Specifically, we solve the following set of equilibrium equations under external illumination from both sides by an isotropic radiation field of specific intensity $J_{v}^{\infty}$ equal to the cosmological $J_{v}$ at each point in the absorber:

$$
\begin{gathered}
\alpha_{\mathrm{HI}}(T) n_{\mathrm{HI}} n_{\mathrm{e}}=\Gamma_{\mathrm{HI}} n_{\mathrm{HI}}, \\
\alpha_{\mathrm{HeI}}(T) n_{\mathrm{He} \text { II }} n_{\mathrm{e}}=\Gamma_{\mathrm{HeI}_{\mathrm{I}}} n_{\mathrm{He} \mathrm{I}}, \text { and } \\
\alpha_{\mathrm{He} \text { II }}(T) n_{\mathrm{He} \text { II }} n_{\mathrm{e}}=\Gamma_{\mathrm{He} \mathrm{II}} n_{\mathrm{He} \text { II }}
\end{gathered}
$$

subject to the constraints $n_{\mathrm{H}}=n_{\mathrm{HI}}+n_{\mathrm{HII}}, n_{\mathrm{He}}=n_{\mathrm{HeI}}+$ $n_{\mathrm{He} \mathrm{II}}+n_{\mathrm{He} \text { III }}$, and $n_{\mathrm{e}}=n_{\mathrm{H} \mathrm{II}}+n_{\mathrm{He} \mathrm{II}}+2 n_{\mathrm{He} \text { III }}$. The photoionization equilibrium assumption is generally accurate as the ionization timescale is much smaller than the Hubble time and collisional processes are negligible at the densities and temperatures considered (Haardt \& Madau 1996).

To close the system of Equations (A1)-(A3), we must specify how to calculate the photoionization rates. Let $J_{v}=$ $(1 / 4 \pi) \int d \Omega I_{v}(\theta)$ be the angle average of the total specific intensity at any given point. Then the local photoionization rate for species $i \in\{\mathrm{HI}, \mathrm{He} \mathrm{I}, \mathrm{He} \mathrm{II}\}$ is given by

$$
\Gamma_{i}(x)=4 \pi \int_{v_{i}}^{\infty} \frac{d v}{h v} \sigma_{i}(v) J_{v}(x) .
$$

\footnotetext{
7 Here, $\gamma$ is the adiabatic index and $\mu$ the mean molecular weight of the gas. These are taken to be $5 / 3$ and 0.59 , respectively, corresponding to a monatomic and fully ionized gas of cosmic composition.
}

The specific intensity along a particular ray will in general depend on the angle $\theta$ of incidence inside the slab because different rays encounter different optical depths. We take the total specific intensity at any given point to be the sum of the radiation originating from the external background and of the radiation from the recombination processes to $\mathrm{HI}_{\mathrm{I}}$ and He II (HI LyC, He II BalC, He II Ly $\alpha$, and He II LyC) within the absorber calculated as in Section 4 and Appendix B. For this purpose, the recombination line photons are treated as $\delta$ functions, which is a good approximation within individual absorbers. Each component is attenuated in magnitude depending on the optical depth from its source following the usual transfer equation.

In particular, our approach explicitly takes into account the coupling between different species arising from the reabsorption of recombination photons by a different species. For example, ionizing $\mathrm{He}$ II LyC recombination photons will in general not only be reabsorbed by He II, but also by $\mathrm{H}$ I and He I. Because of this explicit treatment of recombinations, case A recombination coefficients are appropriate in Equations (A1)-(A3). As He I is found to play a negligible role in our calculations, we do not treat its recombination processes in detail, but we do approximate their effect by using the case B recombination coefficient in the optically thick regime.

Our calculations use 100 spatial bins, 100 logarithmically spaced frequency bins, and 20 angular bins covering $0 \leqslant \theta<$ $\pi \leqslant 2$. The equations are solved iteratively until convergence to better than one part in $10^{3}$ is attained at each point.

\section{APPENDIX B}

\section{RECOMBINATION EMISSION}

Here we provide the technical details of our treatment of recombination emission from individual absorbers outlined in Section 4, including the analytic approximations to the selfconsistent numerical photoionization results.

\section{B.1. General Formalism}

Consider a generic line recombination process with a source function $S_{v}^{\mathrm{rec}} \equiv j_{v}^{\mathrm{rec}} / \alpha_{v}$, where

$$
j_{v}^{\mathrm{rec}}=\frac{h v_{\mathrm{rec}}}{4 \pi} \alpha_{\mathrm{rec}}(T) n_{i+1} n_{e} \phi_{\mathrm{rec}}(v)
$$

is the relevant emission coefficient, and $\alpha_{v}=\sum_{i} n_{i} \sigma_{i}(v)$ is the absorption coefficient accounting for absorption by all species. Here, $v_{\text {rec }}$ is the frequency of the recombination line of interest; we make the approximation that the line is sufficiently narrow so that it can be represented by a single frequency for energetic purposes, and the frequency dependence of the line profile is captured by the function $\phi_{\mathrm{rec}}(v)$, discussed in Section 4.2. The effective recombination coefficient $\alpha_{\text {rec }}(T)$ accounts for all the channels leading to the transition of interest. We use the subscript " $i+1$ " to refer to singly ionized species $i$. For instance, if $i$ is $\mathrm{HI}$, then $i+1$ is $\mathrm{HII}$, and if $i$ is He II, then $i+1$ is He III.

A given ray crossing a slab absorber with incidence angle $\theta$ will emerge with extra recombination photons following the general solution to the radiative transfer equation (e.g., Rybicki \& Lightman 1979):

$$
I_{v}^{\mathrm{rec}}\left(N_{\mathrm{H} I} ; x=\infty, \mu\right)=\int_{0}^{\tau_{v}(x=\infty)} d\left(\tau_{v}^{\prime} / \mu\right) e^{-\left(\tau_{v}-\tau_{\nu}^{\prime}\right) / \mu} S_{v}^{\mathrm{rec}}\left(\tau_{\nu}^{\prime}\right),
$$


where $\tau_{v}$ is the optical depth normal to the slab and $\mu=\cos \theta$. The recombination intensity can also be calculated in this way at any point $x$ within the slab to model the effects of recombinations on the ionization structure of the slab itself (Section 3 and Appendix A). Since $\partial^{2} N / \partial z \partial N_{\mathrm{HI}}$ is the observed column density distribution, the appropriate value of $\mu$ to use in calculating the cosmological emissivity (Equation (8)) is 1, and we define $I_{v}^{\mathrm{rec}}\left(N_{i}\right) \equiv I_{v}^{\mathrm{rec}}\left(N_{i} ; x=\infty, \mu=1\right)$.

In the optically thin limit $\tau_{v}(x=\infty) \ll 1$,

$$
I_{v}^{\mathrm{rec}}\left(N_{\mathrm{HI}}\right) \stackrel{\tau_{v} \ll 1}{\rightarrow} \frac{h v_{\mathrm{rec}}}{4 \pi} \frac{\alpha_{\mathrm{rec}}(T)}{\alpha_{i}^{A}(T)} N_{i} \Gamma_{i} \phi_{\mathrm{rec}}(v),
$$

so that the recombination emission is proportional to the column density of the absorber $\left(N_{i}\right)$, times the number of incident ionizing photons $\left(\Gamma_{i}\right)$, times the fraction of recombinations that lead to the recombination process of interest $\left(\alpha_{\text {rec }}(T) / \alpha_{i}^{A}(T)\right)$. As an absorber becomes optically thick, the number of incident photons it absorbs saturates as $1-e^{-\tau_{v}}$, so that we expect that the recombination emission will also saturate accordingly. As the numerical calculations in Figures 2 and 3 show, this is the case and will be the basis for the analytical approximations to the re-emission that we develop next.

\section{B.2. Analytic Approximations}

A good analytic approximation to the emergent re-emission from an absorber, capturing the optically thin limit and the optically thick saturation, is given by

$$
I_{v}^{\mathrm{rec}}\left(N_{i}\right)=\frac{h v_{\mathrm{rec}}}{4 \pi} \frac{\alpha_{\mathrm{rec}}(T)}{\alpha_{i}^{A}(T)} \min \left(N_{i}, N_{\mathrm{i}, \text { thresh }}\right) \Gamma_{i} \phi_{\mathrm{rec}}(v),
$$

where $N_{\text {thresh }}$ is a threshold column density, near the optically thick transition, at which the recombination intensity saturates. To make the approximation smoothly varying with column density, it is convenient to make the replacement $\min \left(N_{i}, N_{i, \text { thresh }}\right) \rightarrow N_{i, \text { thresh }}\left(1-e^{-N_{i} / N_{i, \text { thresh }}}\right)$, which we do throughout in our numerical evaluations. By inspection, we find that $N_{\mathrm{H} \text {,thresh }}=10^{16.75} \mathrm{~cm}^{-2}$ and $N_{\mathrm{He} \text { II,thresh }}=10^{17.3} \mathrm{~cm}^{-2}$ give good approximations to the full numerical results for the $\mathrm{H}$ I LyC and He II LyC processes, respectively (Figure 2).

For $\mathrm{He}$ II $\mathrm{BalC}$ re-emission, a more robust approximation is obtained by noting that in the optically thick regime, the absorbers have a "skin" (analogous to a region behind an ionizing front) that is nearly uniform in $\mathrm{H}$ I and He II. While the recombinations are to He II, the opacity to the $1 \mathrm{Ryd}$ recombination photons owes to $\mathrm{H}$ I. Because this skin is optically thick at the recombination energy, the emergent intensity simply approaches the source function. The value of the source function near the outer edge of the absorber can be approximated using optically thin photoionization abundance ratios:

$$
\begin{aligned}
& I_{v}^{\mathrm{He} n \mathrm{BBalC}}\left(N_{\mathrm{H}_{\mathrm{I}}}\right) \rightarrow S_{v}^{\mathrm{He} \text { IBalC }} \text { (skin) } \\
& \approx \frac{h v_{\mathrm{He} \mathrm{IBalC}}}{4 \pi} \frac{\alpha_{\mathrm{He} \text { II } n=2}(T)}{\alpha_{\mathrm{He} \mathrm{II}}^{A}(T)} \frac{\eta_{\text {thin }} \Gamma_{\mathrm{He} \mathrm{II}}^{\mathrm{ext}}}{\sigma_{\mathrm{HI}}\left(v_{\mathrm{HI}}\right)} \phi_{\mathrm{rec}}(v) .
\end{aligned}
$$

For this process, we approximate the re-emission as the minimum of the optically thin limit and this optically thick result.

As Ly $\alpha$ is a resonant transition, its recombination photons will scatter until they diffuse out of the absorber unless they are destroyed by dust, metals, or $\mathrm{H}$ I continuum opacity in the case of $\mathrm{He}$ II $\mathrm{Ly} \alpha$ which can ionize it. Of particular interest for the destruction by metals is the coincidence between the O III $\lambda 303.799$ line and the He II Ly $\alpha$ line at $303.783 \AA$ (Bowen 1934). Studies of this process (including H I opacity) in the context of dense and enriched planetary nebulae and galactic nuclei (Weymann \& Williams 1969; Kallman \& McCray 1980; Eastman \& MacAlpine 1985) suggest that the vast majority of the He II Ly $\alpha$ emission created by recombinations diffuse unimpeded into the IGM for Ly $\alpha$ forest systems. Only the most metal-rich Lyman limit systems and damped Ly $\alpha$ absorbers potentially lose a significant fraction of their He II Ly $\alpha$ recombination photons to O III, which should have little global impact. Similarly, only the highest column density and most chemically evolved systems are likely to contain enough dust to efficiently destroy H I Ly $\alpha$ photons. We therefore adopt a simplified treatment of $\mathrm{HI}$ and $\mathrm{He}$ II Ly $\alpha$ recombination emission in which we assume that all recombination photons escape into the IGM. Instead of integrating the source function over the absorber, we thus simply integrate the emission coefficient $j_{v}{ }^{\mathrm{H} / \mathrm{He}}{ }^{\mathrm{LLy} \alpha}$ :

$$
I_{v}^{\mathrm{H} \mathrm{I} / \mathrm{He} \mathrm{II} \mathrm{Ly} \alpha}\left(N_{\mathrm{HI}}\right)=\int_{-\infty}^{\infty} d x j_{\nu}^{\mathrm{H} / \mathrm{He}{ }^{\mathrm{LL}} \alpha}(x) .
$$

By the preceding arguments, this is likely a reasonable approximation, but certainly an upper bound. We assume case B conditions in which higher Lyman-series photons are ultimately degraded into lower energy photons, with the ultimate production of extra Ly $\alpha$ photons, which we implement by taking the appropriate case B emission coefficient.

For the Ly $\alpha$ processes, particularly good approximations to the numerical results can be developed, as the optically thick limit can be accurately estimated. The key observation is that the fraction of recombinations ultimately leading to the re-emission of a $\operatorname{Ly} \alpha$ photon is a fixed number (at a given temperature) equal to the sum of all the production channels allowed by the selection rules. Recombinations directly to $n=1$ produce LyC photons, but unless the recombination occurs near the skin of the absorber, this photon will be reabsorbed before escaping and one must account for the probability that it will ultimately result in a Ly $\alpha$ photon. Averaging over angles, the number of ionizing photons incident from one side of the absorber that are absorbed per unit time per unit area per unit solid angle is given by

$$
\begin{aligned}
\dot{N}_{\mathrm{abs}}^{i}\left(N_{\mathrm{HI}}\right) & =\int_{0}^{1} d \mu \int_{v_{i}}^{\infty} \frac{d v}{h v} J_{\nu}^{\infty}\left[1-e^{\tau_{\nu} / \mu}\right] \\
& =\int_{v_{i}}^{\infty} \frac{d v}{h v} J_{v}^{\infty}\left[1-\tau_{\nu} \Gamma\left(-1, \tau_{\nu}\right)\right],
\end{aligned}
$$

where $\Gamma(.,$.$) is the upper incomplete gamma function and$ is unrelated to the photoionization rate, and $i \in\left\{\mathrm{H}_{\mathrm{I}}, \mathrm{He}_{\mathrm{I}}\right\}$ indicates the species of interest. Since in equilibrium the total number of recombinations equals the number of ionizations, the Ly $\alpha$ re-emission in the very optically thick regime can then be written as

$$
\begin{aligned}
& I_{\nu}^{\mathrm{H} \mathrm{I} / \mathrm{He}{ }_{\mathrm{II}} \mathrm{Ly} \alpha}\left(N_{\mathrm{H}_{\mathrm{I}}}\right)=f_{\alpha}^{\text {thick }} h v_{\mathrm{H} \mathrm{I} / \mathrm{He}{ }_{\mathrm{IIL}} \mathrm{Ly} \alpha} \dot{N}_{\mathrm{abs}}^{\mathrm{HI} / \mathrm{He}{ }_{\mathrm{II}} \mathrm{Ly} \alpha}\left(N_{\mathrm{HI}}\right) \\
& \times \delta\left(v-v_{\mathrm{H} / \mathrm{He}}{ }_{\mathrm{IL}} \mathrm{L} \alpha\right),
\end{aligned}
$$

where $f_{\alpha}^{\text {thick }}=0.4$, set by matching the numerical calculations (Figure 3), accounts for the efficiency of Ly $\alpha$ photon production and geometrical effects. In general, we again approximate the 
re-emission as the minimum between the optically thin and optically thick results.

Note that an alternative approach for approximating the recombination emission can be derived from the fact that the cosmological emissivity (Equation (8)) ultimately does not depend on the detailed function $I_{v}^{\text {rec }}\left(N_{i}\right)$ but on its integral over the column density distribution. The part of the integral involving $N_{i}$ can thus be factored out and pre-computed for a given column density distribution. Fardal et al. (1998) used an approach along these lines, and we also make use of this fact in the following section (Equations (C5) and (C7)) in developing an analytic model for the integrated recombination contribution to the ionizing background.

\section{APPENDIX C \\ ANALYTIC MODEL FOR THE RECOMBINATION CONTRIBUTION TO THE IONIZING BACKGROUND}

Here we provide a quantitative analytic basis for understanding the full numerical results on the contribution of recombinations to the ionizing background obtained in Section 6.2. We focus on the H I photoionization rate and assume that the mean free path is sufficiently short that the local source approximation of Equation (D1) applies, which is valid at $z \gtrsim 2$. Based on Section 4 and in particular Figure 2, we assume that $\mathrm{H} \mathrm{I} \mathrm{LyC}$ re-emission is the dominant process. Following the notation of the main text, let us define $\Gamma_{\mathrm{H}_{\mathrm{I}}}^{\text {withrec }} \equiv \Gamma_{\mathrm{HI}}^{\text {norec }}+\Gamma_{\mathrm{HI}_{\mathrm{I}}}^{\mathrm{rec}}$ so that $\Gamma_{\mathrm{HI}}^{\mathrm{rec}}$ is the portion of the photoionization rate that is contributed by recombinations.

As outlined in Section 6.2, the recombination contribution depends on several factors. Quantitatively, we write

$$
\frac{\Gamma_{\mathrm{HI}}^{\mathrm{rec}}}{\Gamma_{\mathrm{HI}}^{\mathrm{withrec}}}=\left.\frac{\Gamma_{\mathrm{HI}}^{\mathrm{rec}}}{\Gamma_{\mathrm{HI}}^{\text {withrec }}}\right|_{\max } \times f_{\text {leak }} \times f_{\sigma_{\mathrm{i}}}
$$

where the first term is the maximum that would be attained if all the re-emitted photons contributed to the ionizing background and all had frequency $v_{\mathrm{H}_{\mathrm{I}}}, f_{\text {leak }}$ accounts for the leakage of the photons below the ionization edge, and $f_{\sigma_{\mathrm{i}}}$ accounts for the frequency dependence of the photoionization cross section and recombination line profile.

We first consider the maximum contribution by assuming that a negligible fraction of the recombination photons leak below the ionization edge before being reabsorbed and taking the recombination line profile to be a pure $\delta$ function, $\epsilon_{v}^{\text {rec }} \equiv$ $\tilde{\epsilon}_{v_{\mathrm{HI}}}^{\mathrm{rec}} \delta\left(v-v_{\mathrm{HI}}\right)$. Then,

$$
\Gamma_{\mathrm{HI}}^{\mathrm{rec}}=4 \pi \int_{\nu_{\mathrm{HI}}}^{\infty} \frac{d v}{h v} J_{v}^{\mathrm{rec}} \sigma_{\mathrm{HI}}(v)=\frac{\Delta l_{\mathrm{mfp}}\left(v_{\mathrm{HI}_{\mathrm{I}}}\right) \sigma_{\mathrm{HI}} \tilde{\epsilon}_{\nu_{\mathrm{HI}}}^{\mathrm{rec}}}{h v_{\mathrm{HI}}} .
$$

Combining Equations (8) and (B4) for the cosmological recombination emissivity and the special case of $\mathrm{H}$ i LyC gives

$$
\begin{aligned}
\tilde{\epsilon}_{\nu_{\mathrm{HI}}}^{\mathrm{rec}}= & h \nu_{\mathrm{HI}} \frac{\alpha_{\mathrm{H}, \mathrm{n}=1}(T)}{\alpha_{\mathrm{H} \mathrm{I}}^{\mathrm{A}}(T)} \Gamma_{\mathrm{HI}}^{\mathrm{withrec}} \frac{d z}{d l} \\
& \times \int_{0}^{\infty} d N_{\mathrm{HI}} \frac{\partial^{2} N}{\partial z \partial N_{\mathrm{HI}}} N_{\mathrm{H} \text { I,thresh }}\left(1-e^{-N_{\mathrm{HI}} / N_{\mathrm{HI}, \text { thresh }}}\right)
\end{aligned}
$$

and therefore

$$
\left.\frac{\Gamma_{\mathrm{HI}}^{\mathrm{rec}}}{\Gamma_{\mathrm{HI}}^{\text {withrec }}}\right|_{\max }=\frac{\alpha_{\mathrm{HI}, \mathrm{n}=1}(T)}{\alpha_{\mathrm{H}_{\mathrm{I}}}^{\mathrm{A}}(T)} f_{\mathrm{eff}},
$$

where

$$
\begin{aligned}
f_{\text {eff }} \equiv & \Delta l_{\text {mfp }}\left(v_{\mathrm{H}_{\mathrm{I}}}\right) \sigma_{\mathrm{H}_{\mathrm{I}}} \frac{d z}{d l} \\
& \times \int_{0}^{\infty} d N_{\mathrm{H} \mathrm{I}} \frac{\partial^{2} N}{\partial z \partial N_{\mathrm{H}}} N_{\mathrm{H}_{\mathrm{I}}, \text { thresh }}\left(1-e^{-N_{\mathrm{HI}} / N_{\mathrm{H}, \text { thresh }}}\right)
\end{aligned}
$$

is a dimensionless efficiency factor whose value depends on how recombination emission that saturates with column density relative to absorption (the mean free path term). Using Equations (3) and (D2), we can express the mean free path at the ionization edge as

$$
\Delta l_{\mathrm{mfp}}\left(\nu_{\mathrm{HI}}\right)=\frac{d l}{d z}\left[\int_{0}^{\infty} d N_{\mathrm{HI}} \frac{\partial^{2} N}{\partial z \partial N_{\mathrm{HI}}}\left(1-e^{-\sigma_{\mathrm{HI}} N_{\mathrm{HI}}}\right)\right]^{-1}
$$

and thus

$$
f_{\text {eff }}=\frac{\int_{0}^{\infty} d N_{\mathrm{HI}_{\mathrm{I}}} \partial^{2} N / \partial z \partial N_{\mathrm{HI}}\left(\sigma_{\mathrm{HI}_{\mathrm{I}}} N_{\mathrm{HI}, \text { thresh }}\right)\left(1-e^{-N_{\mathrm{HI}} / N_{\mathrm{HI}, \text { thresh }}}\right)}{\int_{0}^{\infty} d N_{\mathrm{HI}} \partial^{2} N / \partial z \partial N_{\mathrm{HI}}\left(1-e^{-\sigma_{\mathrm{HI}} N_{\mathrm{HI}}}\right)} .
$$

For a power-law column density distribution as in Equation (5), the redshift dependence cancels out in the ratio and the integrals over $N_{\mathrm{H}}$ can be done analytically in terms of gamma functions. The latter also cancel out, leaving a very simple result

$$
f_{\text {eff }}=\left(\sigma_{\mathrm{HI}} N_{\mathrm{H} \mathrm{I}, \text { thresh }}\right)^{2-\beta} \text {. }
$$

In Section B.2, we found that $N_{\mathrm{HI}, \text { thresh }} \approx 10^{16.75} \mathrm{~cm}^{-2}$ provides a good approximation for $\mathrm{H}_{\mathrm{I}}$ LyC. Since $\sigma_{\mathrm{HI}} N_{\mathrm{H} \text {, thresh }}<1$, Equation (C8) shows quantitatively how the recombination contribution increases with the steepness of the column density distribution (large $\beta$ ). As noted in Section 6.2, this physically arises because a larger fraction of recombinations occur in optically thin absorbers which return more of their recombination photons into the IGM.

For a hydrogenic atom of integer charge $Z$, the fraction of recombinations that are to level $n$ is

$$
\frac{\alpha_{\mathrm{H}, \mathrm{n}}(T)}{\alpha_{\mathrm{H} \mathrm{I}}^{\mathrm{A}}(T)}=\frac{n^{-3} e^{\phi_{n} / k T} \operatorname{Ei}\left(\phi_{n} / k T\right)}{\sum_{i=1}^{\infty} i^{-3} e^{\phi_{i} / k T} \operatorname{Ei}\left(\phi_{i} / k T\right)},
$$

where $\phi_{i}=Z^{2} h v_{\mathrm{HI}} / i^{2}$ is the ionization energy of level $i$ and $E i(x) \equiv \int_{x}^{\infty} d x^{\prime} e^{-x^{\prime}} / x^{\prime}$ (Cillié 1932).

The actual contribution of recombinations to the ionizing background is smaller because many recombination photons rapidly redshift below the ionization threshold. What fraction of photons are lost through this leakage? Focusing again on LyC recombinations, a photon is re-emitted just above the ionization edge with energy $v^{\prime}=v_{\mathrm{H}_{\mathrm{I}}}+\Delta v$, where $\Delta v / \nu_{\mathrm{H}_{\mathrm{I}}} \ll 1$. Supposing this photon is re-emitted at redshift $z_{\text {rec }}$, it will redshift below $v_{\mathrm{HI}}$ after traveling a proper distance

$$
\Delta l_{\text {leak }}\left(z_{\text {rec }} ; \Delta v\right) \approx \frac{d l}{d z}\left(z_{\text {rec }}\right) \frac{\Delta v}{v_{\mathrm{H} \text { I }}}\left(1+z_{\text {rec }}\right) .
$$

The local source approximation of Equation (D1) is valid because in this regime we can write $J_{v}=(4 \pi)^{-1} \int_{0}^{\infty} d l \epsilon_{v} e^{-l / \Delta l_{\mathrm{mfp}}}$, with the emissivity treated as a constant. The leakage of recombination photons owing to redshifting implies that the emissivity should really integrated over a maximum distance $\Delta l_{\text {leak }}$ :

$$
J_{v}^{\mathrm{rec}}=\frac{1}{4 \pi} \int_{0}^{\Delta l_{\text {leak }}} d l \epsilon_{v}^{\mathrm{rec}} e^{-l / \Delta l_{\mathrm{mfp}}}=\frac{1}{4 \pi} \Delta l_{\mathrm{mfp}} \epsilon_{v}^{\mathrm{rec}}\left[1-f_{\text {lost }}\right],
$$



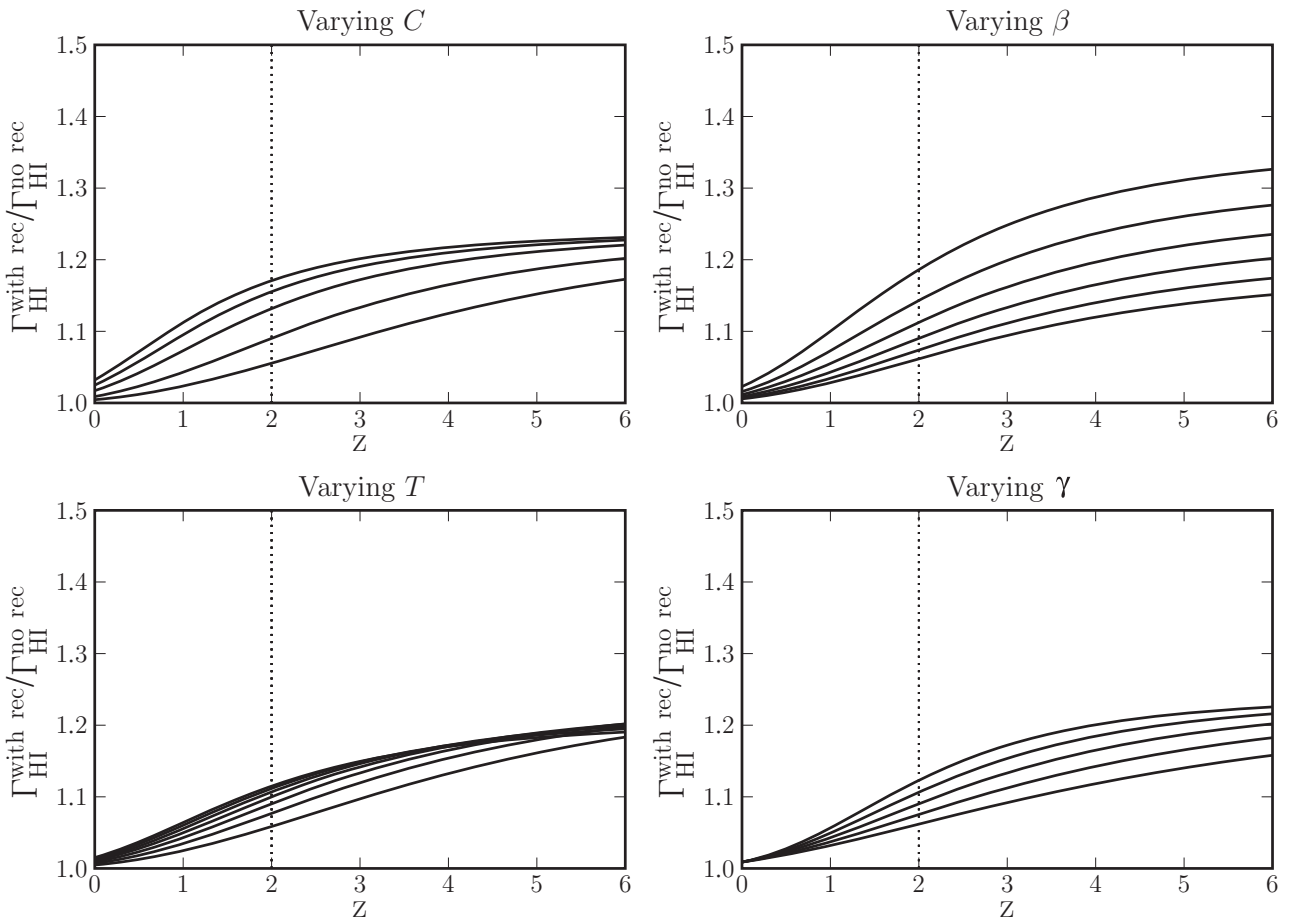

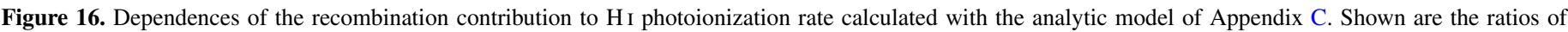

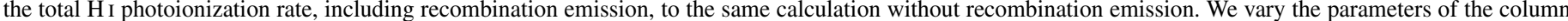

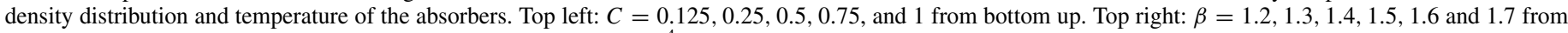

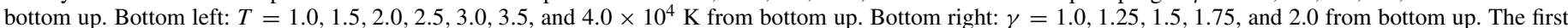

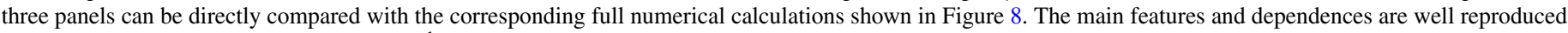

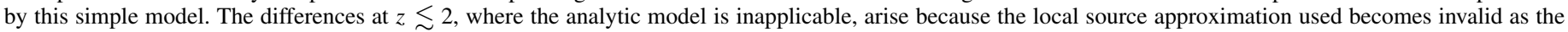
radiation field begins to be limited by the cosmological horizon.

where $f_{\text {lost }}\left(z_{\text {rec }} ; \Delta v\right) \equiv e^{-\Delta l_{\text {leak }} / \Delta l_{\text {mfp }}}$ is the fraction of recombination photons emitted with frequency above the ionization edge that are lost to redshifting.

The overall fraction of photons lost is then an average over the recombination line profile:

$$
f_{\text {lost }}\left(z_{\text {rec }}\right)=\int_{v_{\mathrm{HI}}}^{\infty} d v^{\prime} \phi_{\text {rec }}\left(v^{\prime}\right) f_{\text {lost }}\left(z_{\text {rec }} ; \Delta v=v^{\prime}-v_{\mathrm{HI}}\right) .
$$

The result is simplified if the line profile is taken to be purely exponential, $\phi_{\mathrm{rec}}\left(v^{\prime}\right)=(h / k T) e^{-h \Delta v / k T}$ for $\Delta v \geqslant 0$, in which case

$$
f_{\text {lost }}\left(z_{\text {rec }}\right)=\frac{1}{1+y\left(z_{\text {rec }} ; \Delta l_{\mathrm{mfp}}, T\right)},
$$

where

$$
y\left(z_{\mathrm{rec}} ; \Delta l_{\mathrm{mfp}}, T\right) \equiv \frac{k T / h v_{\mathrm{HI}}}{\Delta l_{\mathrm{mfp}}(d z / d l) /\left(1+z_{\mathrm{rec}}\right)} .
$$

The mean free path depends on redshift and on the parameters of the column density distribution. Using the analytical expression (D3) for $\Delta l_{\mathrm{mfp}}$, we can express $f_{\text {lost }}$ directly in terms of these basic parameters

$$
f_{\text {lost }}\left(z_{\mathrm{rec}} ; C, \gamma, \beta, T\right)=\frac{1}{1+C \Gamma(2-\beta)\left(1+z_{\mathrm{rec}}\right)^{1+\gamma} k T / h \nu_{\mathrm{H}}},
$$

and finally define $f_{\text {leak }} \equiv 1-f_{\text {lost }}$.

The last term to consider is the suppression factor that arises because the photoionization cross section entering in the photoionization rate from recombinations is frequency dependent, and photons are really re-emitted with finite energy above the ionization threshold. This is simply a frequency average of the cross section, over its maximum at $\nu_{\mathrm{H}}$ :

$$
\begin{aligned}
f_{\sigma_{i}} & =\int_{v_{\mathrm{HI}}}^{\infty} d v^{\prime} \phi_{\mathrm{rec}}\left(v^{\prime}\right)\left(v / \nu_{\mathrm{HI}}\right)^{-3} \\
& =\left(\frac{h v_{\mathrm{HI}}}{k T}\right)^{3} e^{-h \nu_{\mathrm{HI}} / k T} \Gamma\left(-2, h v_{\mathrm{HI}} / k T\right),
\end{aligned}
$$

where we have again approximated the recombination radiation to have a purely exponential profile and that the cross section scales as $v^{-3}$ just above the ionization edge.

In Figure 16, we combine these analytic results and show how $\Gamma_{\mathrm{HI}}^{\text {withrec }} / \Gamma_{\mathrm{HI}}^{\text {norec }}=1 /\left(1-\Gamma_{\mathrm{HI}}^{\text {rec }} / \Gamma_{\mathrm{HI}}^{\text {withrec }}\right)$ varies as a function of the parameters of the column density distribution and the temperature of the absorbers. Note that the main features and dependences of the corresponding full numerical calculations shown in Figure 8 are well reproduced by this simple model. The differences at $z \lesssim 2$ arise because the local source approximation used in the analytic calculations becomes invalid as the radiation field begins to be limited by the cosmological horizon; the "effective mean free path" is then shorter than the mean absorption distance and more recombination photons are thus in reality retained in the ionizing range.

\section{APPENDIX D}

\section{SPECTRAL FILTERING}

The spectral index of a radiation background in general differs from the spectral index of its sources owing to filtering along the line of sight. In this section, we explore different 
filtering cases relevant to the ionizing background in order to provide physical understanding of our numerical solutions of the radiative transfer equation and provide analytical results referred to in the main text.

An important characteristic of the ionizing background at high redshifts is that it is local in the sense that the specific intensity $J_{v}$ depends only on the local value of the specific emissivity $\epsilon_{v}$ :

$$
J_{v}(z) \approx \frac{1}{4 \pi} \Delta l_{\mathrm{mfp}}(v, z) \epsilon_{v}(z) .
$$

Here, $\Delta l_{\mathrm{mfp}}(v, z)$ is the mean free path of photons of frequency $v$ and redshift $z$ and is given by

$$
\Delta l_{\mathrm{mfp}}\left(v_{0}, z_{0}\right)=\frac{d l}{d z}\left(z_{0}\right)\left(\frac{d \bar{\tau}\left(v_{0}, z_{0}, z\right)}{d z}\right)^{-1}\left(z_{0}\right)
$$

This limit of Equation (2) is valid whenever photons are absorbed so close to their point of emission that they redshift only negligibly. For H I ionizing photons of wavelength $912 \AA$, the "breakthrough" point above which this approximation holds is approximately $z=2$ (Madau et al. 1999). In this regime, cosmological effects are unimportant and calculations can be performed in ordinary Euclidean geometry. Then the effective optical depth at frequency $v$ over a proper length $l$ at redshift $z$ can be written as $\bar{\tau}(\nu, z, l)$ and $\Delta l_{\mathrm{mfp}}(\nu, z)=(d \bar{\tau} / d l)^{-1}$. A useful analytical expression for the mean free path can be obtained at frequencies where the photoionization cross section $\sigma_{i} \propto v^{-3}$ and the column density distribution is described by single power laws $\beta$ and $\gamma$ (see Equation (5)). In the case of $\mathrm{HI}$,

$$
\begin{aligned}
\Delta l_{\mathrm{mfp}}\left(v_{0}, z_{0}\right) \approx & \frac{(\beta-1) c}{\Gamma(2-\beta) N_{0} \sigma_{\mathrm{HI}}^{\beta-1}}\left(\frac{v_{0}}{v_{\mathrm{HI}}}\right)^{3(\beta-1)} \\
& \times \frac{1}{\left(1+z_{0}\right)^{\gamma+1} H\left(z_{0}\right)}
\end{aligned}
$$

and it is straightforwardly generalized to any other single ion.

An implicit assumption in Equation (D1) (as well as throughout much of this paper, such as in Equations (1) and (2)) is that a sphere of radius one mean free path contains sufficiently many sources that their effect is well captured by the use of a volumeaveraged uniform emissivity. This is generally valid when the sources are star-forming galaxies, which are very numerous. Quasars, however, are much rarer and this assumption in general fails. (See Figure 9 and the discussion in Section 7.1.) We will therefore also consider the case of filtering of radiation from an isolated source. For each case, we will consider both the cases in which the intervening absorbers are uniformly distributed and the one in which discrete absorbers are Poisson-distributed following a column density distribution.

\section{D.1. Uniform Emissivity and Absorbing Material}

In the case in which both the emissivity and the absorbing material are spatially uniform,

$$
\begin{aligned}
J_{v}(z) & \approx \frac{1}{4 \pi} \Delta l_{\mathrm{mfp}}(v, z) \epsilon_{v}(z) \text { with } \bar{\tau}(v, z, l) \\
& =n_{\mathrm{abs}} \sigma_{\mathrm{abs}}(v) l, \text { so that } J_{v}(z) \approx \frac{1}{4 \pi} \frac{\epsilon_{v}(z)}{n_{i} \sigma_{i}(v)},
\end{aligned}
$$

where $n_{i}$ is the number density of the absorbing material, and $\sigma_{i}(v)$ is its cross section. For photons with $\nu_{\mathrm{H}_{\mathrm{I}}} \leqslant v<$
$\nu_{\mathrm{He} \text { II }}$, the dominant source of continuum opacity owes to $\mathrm{HI}$ photoionization, so $n_{i}=n_{\mathrm{HI}}$ and $\sigma_{i}(v)=\sigma_{\mathrm{H}_{\mathrm{I}}}(v)$. For $v \geqslant v_{\mathrm{He} \text { II }}$, $\mathrm{H}$ I continuum opacity is fractionally small in the cosmological context and we can take $n_{i}=n_{\mathrm{He} \text { II }}$ and $\sigma_{i}(v)=\sigma_{\mathrm{He} \text { II }}(\nu)$. Since both absorbing ions are hydrogenic, they similarly harden spectra following $J_{v} \propto \epsilon_{\nu} / \sigma_{i}(v) \approx \epsilon_{v} v^{3}$. The last equality is approximately valid near the ionization edge. At high energies, the photoionization cross section decreases more slowly and the hardening becomes negligible.

\section{D.2. Uniform Emissivity and Discrete Absorbers}

If the emissivity is spatially uniform, but the absorbers are discrete and Poisson-distributed, the result is similar, but with the effectively optical depth calculated from the column density distribution as in Equation (3). If the column density distribution of the absorbers follow a power-law $d N / d N_{i} \propto N_{i}^{-\beta}$, then just above the ionization edge where the cross section $\sigma_{i}(v) \propto v^{-3}$, the mean free path $\Delta l_{\mathrm{mfp}}(\nu, z) \propto v^{3(\beta-1)}$ (Zuo \& Phinney 1993; Faucher-Giguère et al. 2008b) and therefore $J_{v} \propto \epsilon_{v} v^{3(\beta-1)}$. For H I, we may take $\beta=1.4$, as measured by Misawa et al. (2007), in which case the hardening $\alpha \rightarrow \alpha-1.2$ is significantly weaker than the $\alpha \rightarrow \alpha-3$ in the uniform absorbing material case above the $\mathrm{H}$ i ionization edge. If the proportionality factor $\eta$ between $N_{\mathrm{H} \text { I }}$ and $N_{\mathrm{He} \text { II }}$ were a constant throughout, the radiation above the He II ionization edge would be identically hardened. However, the complex behavior of $\eta$ in the optically thick regime (Section 3.2) may alter this result; this behavior is taken into account in our numerical calculations.

\section{D.3. Point Source and Uniform Absorbing Material}

The case of an isolated point source is quite different. In this case, the specific intensity (which can no longer be assumed to be isotropic, so we denote it by $I_{v}$ instead of $J_{v}$ ), is exponentially suppressed with increasing optical depth from the source

$$
I_{v}=I_{v}(l=0) e^{-\tau_{v}(l)} .
$$

We again assume that $\mathrm{H}_{\mathrm{I}}$ continuum opacity dominates for $v_{\mathrm{H} \text { I }} \leqslant v<v_{\text {He II }}$ and that He II continuum opacity dominates for $v>v_{\mathrm{He} \text { II }}$. In each regime, $\tau_{v}(l)=n_{i} \sigma_{i}(v) l$. The spectrum is attenuated relative to its value at $\nu_{\mathrm{i}}$ by a factor

$$
\begin{aligned}
\frac{I_{\nu}}{I_{v_{i}}} & =\frac{e^{-\tau_{v}}}{e^{-\tau_{v_{i}}}}=e^{\tau_{v_{i}}} e^{-\tau_{v_{i}}\left[\sigma_{i}(v) / \sigma_{i}\left(v_{i}\right)\right]} \propto\left(e^{-\tau_{v_{i}}}\right)^{\sigma_{i}(\nu) / \sigma_{i}\left(v_{i}\right)} \\
& \left.\approx\left(e^{-\tau_{v_{i}}}\right)^{\left(\nu / v_{i}\right.}\right)^{-3}
\end{aligned}
$$

where the last equality again holds approximately just above the ionization edge.

\section{D.4. Point Source and Discrete Absorbers}

For a point source attenuated by Poisson-distributed discrete absorbers, we simply replace the ordinary optical depth by the effective optical depth to obtain the average spectrum:

$$
I_{v}(l)=I_{v}(l=0) e^{-\bar{\tau}_{v}(l)} .
$$

Drawing on our previous results,

$$
\frac{I_{\nu}}{I_{\nu_{i}}}=\frac{e^{-\bar{\tau}_{v}}}{e^{-\bar{\tau}_{v_{i}}}}=e^{\bar{\tau}_{\nu_{i}}} e^{-\bar{\tau}_{\nu}} \propto e^{-\bar{\tau}_{\nu}} \approx\left(e^{-\bar{\tau}_{v_{i}}}\right)^{\left(\nu / \nu_{\mathrm{i}}\right)^{-3(\beta-1)}} .
$$

It is worth noting, as explained in Section 7.1.1, that at fixed optical depth from a source the hardening is the same regardless 
of how (smoothly or discretely) the intervening material is distributed. The different average hardening differs in the discrete case really because of the added stochastic nature of the intervening optical depth at fixed distance.

\section{APPENDIX E}

\section{ATOMIC PHYSICS}

The radiative transfer calculations in this paper are ultimately rooted in atomic physics. The recombination rates and $\mathrm{He} \mathrm{I}$ photoionization cross sections are taken from the appendix of Hui \& Gnedin (1997). For the Ly $\alpha$ emission coefficients and the $\mathrm{HI}$ and $\mathrm{He}$ II photoionization cross sections we take the expressions given by Osterbrock \& Ferland (2006). In particular, given the prominent role it plays in our calculations, the photoionization cross section of a hydrogenic atom of atomic number $Z$ ( $Z=1$ and 2 for $\mathrm{H}_{\mathrm{I}}$ and $\mathrm{He}$ II $)$ is given by

$$
\sigma_{i}(\nu)=\frac{A_{0}}{Z^{2}}\left(\frac{\nu_{1}}{\nu}\right)^{4} \frac{\exp \left\{4-\left[4 \tan ^{-1} \epsilon\right] / \epsilon\right\}}{1-\exp (-2 \pi / \epsilon)}
$$

for $v \geqslant v_{1}$ and 0 otherwise. Here,

$$
A_{0}=\frac{2^{9} \pi}{3 e^{4}} \alpha \pi a_{0}^{2}=6.30 \times 10^{-18} \mathrm{~cm}^{2},
$$

where $\alpha$ is the fine structure constant, and $a_{0}$ is the Bohr radius, $\epsilon=\sqrt{v / v_{1}-1}$, and $h v_{1}=Z^{2} h v_{\mathrm{H}_{\mathrm{I}}}=13.6 Z^{2} \mathrm{eV}$. Just above the photoionization edge $v_{1}$, i.e., for $\epsilon \ll 1, \sigma_{i}(v) \propto v^{-3}$, but the cross section drops more slowly as $v \rightarrow \infty$, explaining the lack of spectral hardening in this limit.

Also of interest is the recombination line profile for freebound transitions, which is important in determining the contribution of recombination emission to the ionizing background (Section 4). The probability that a recombination yields a continuum photon of frequency between $v$ and $v+d v$, by definition, is $\phi_{\text {rec }}(v) d v$. The velocity $u$ of the recombining electron relative to the nucleus ${ }^{8}$ is related to the frequency by $h v=m_{e} u^{2} / 2+h v_{\text {rec }}$, where $v_{\text {rec }}$ is the ionization edge frequency. Now, the probability that the recombining electron has velocity between $u$ and $u+d u$ scales as the probability that an electron in this velocity range recombines when it encounters a nucleus, times the number of electrons with velocity in this range. If $\sigma_{\mathrm{rec}}(u)$ is the velocity-dependent recombination cross section, then the first term is $\propto \sigma_{\text {rec }}(u) u$. In thermal equilibrium, the second term is given by the Maxwell-Boltzmann speed distribution, $f_{\mathrm{M}-\mathrm{B}}(u) \propto u^{2} \exp \left(-m_{e} u^{2} / 2 k T\right)$. Thus,

$$
\phi_{\mathrm{rec}}(v) d v \propto \sigma_{\mathrm{rec}}(u) u f_{\mathrm{M}-\mathrm{B}}(u) d u .
$$

The Milne detailed balance relation relates the recombination cross section to the corresponding photoionization cross section:

$$
\sigma_{\text {rec }}(u)=2\left(\frac{h v}{m_{e} u c}\right)^{2} \sigma_{i}(v)
$$

(e.g., Osterbrock \& Ferland 2006). Using the approximation $\sigma_{i}(v) \propto v^{-3}$, we can solve and find, after normalizing,

$$
\phi_{\mathrm{rec}}(v)=\frac{\left(v / v_{\mathrm{rec}}\right)^{-1} \exp (-h v / k T)}{\Gamma\left(0, h v_{\mathrm{rec}} / k T\right)} \frac{\theta\left(v-v_{\mathrm{rec}}\right)}{v_{\mathrm{rec}}} .
$$

\footnotetext{
8 Note that since $m_{p} / m_{e} \approx 2000$, the nuclei can be assumed to be at rest in
} the frame of the gas.
This profile will also be thermal broadened and shifted owing to the peculiar velocity of the emitting gas; these corrections are however negligible in comparison to the width of the recombination line profile. For instance, the recombination line width $\Delta v_{\text {rec }} / v_{\text {rec }} \approx k T / h v_{\text {rec }} \approx 0.13$ for H I LyC at $T=2 \times 10^{4} \mathrm{~K}$, while the Doppler broadening at the same temperature $\Delta v_{D} / v_{\text {rec }} \approx 0.002$ and the peculiar velocity shift $\Delta v_{\mathrm{pec}} / \nu_{\mathrm{rec}} \approx 0.0003$ for $v_{\mathrm{pec}}=100 \mathrm{~km} \mathrm{~s}^{-1}$.

\section{REFERENCES}

Abel, T., \& Haehnelt, M. G. 1999, ApJ, 520, L13

Aguirre, A., Dow-Hygelund, C., Schaye, J., \& Theuns, T. 2008, ApJ, 689, 851 Aguirre, A., Schaye, J., Kim, T.-S., Theuns, T., Rauch, M., \& Sargent, W. L. W. 2004, ApJ, 602, 38

Barger, A. J., Cowie, L. L., Mushotzky, R. F., Yang, Y., Wang, W.-H., Steffen, A. T., \& Capak, P. 2005, AJ, 129, 578

Becker, G. D., Rauch, M., \& Sargent, W. L. W. 2007, ApJ, 662, 72

Bianchi, S., Cristiani, S., \& Kim, T.-S. 2001, A\&A, 376, 1

Boksenberg, A., Sargent, W. L. W., \& Rauch, M. 2003, arXiv:astro-ph/0307557 Bolton, J., Meiksin, A., \& White, M. 2004, MNRAS, 348, L43

Bolton, J. S., \& Haehnelt, M. G. 2007, MNRAS, 382, 325

Bolton, J. S., Haehnelt, M. G., Viel, M., \& Carswell, R. F. 2006, MNRAS, 366, 1378

Bolton, J. S., Haehnelt, M. G., Viel, M., \& Springel, V. 2005, MNRAS, 357, 1178

Bolton, J. S., Oh, S. P., \& Furlanetto, S. R. 2009, MNRAS, 395, 736

Bouwens, R. J., Illingworth, G. D., Franx, M., \& Ford, H. 2007, ApJ, 670, 928

Bowen, I. S. 1934, PASP, 46, 146

Boyle, B. J., Shanks, T., Croom, S. M., Smith, R. J., Miller, L., Loaring, N., \& Heymans, C. 2000, MNRAS, 317, 1014

Brown, M. J. I., et al. 2006, ApJ, 638, 88

Bruzual, G., \& Charlot, S. 2003, MNRAS, 344, 1000

Bryan, G. L., \& Machacek, M. E. 2000, ApJ, 534, 57

Burles, S., Nollett, K. M., \& Turner, M. S. 2001, ApJ, 552, L1

Cillié, G. 1932, MNRAS, 92, 820

Clegg, R. E. S., \& Middlemass, D. 1987, MNRAS, 228, 759

Cowie, L. L., Barger, A. J., \& Trouille, L. 2009, ApJ, 692, 1476

Cowie, L. L., Songaila, A., Kim, T.-S., \& Hu, E. M. 1995, AJ, 109, 1522

Croft, R. A. C. 2004, ApJ, 610, 642

Croft, R. A. C., Weinberg, D. H., Bolte, M., Burles, S., Hernquist, L., Katz, N., Kirkman, D., \& Tytler, D. 2002, ApJ, 581, 20

Croft, R. A. C., Weinberg, D. H., Pettini, M., Hernquist, L., \& Katz, N. 1999, ApJ, 520, 1

Croom, S. M., Smith, R. J., Boyle, B. J., Shanks, T., Miller, L., Outram, P. J., \& Loaring, N. S. 2004, MNRAS, 349, 1397

Davé, R., Hernquist, L., Katz, N., \& Weinberg, D. H. 1999, ApJ, 511, 521

Dijkstra, M., Haiman, Z., \& Spaans, M. 2006, ApJ, 649, 14

Dunkley, J., et al. 2009, ApJS, 180, 306

Eastman, R. G., \& MacAlpine, G. M. 1985, ApJ, 299, 785

Efstathiou, G. 1992, MNRAS, 256, 43P

Fan, X., Carilli, C. L., \& Keating, B. 2006a, ARA\&A, 44, 415

Fan, X., et al. 2004, AJ, 128, 515

Fan, X., Narayanan, V. K., Strauss, M. A., White, R. L., Becker, R. H., Pentericci, L., \& Rix, H.-W. 2002, AJ, 123, 1247

Fan, X., et al. 2006b, AJ, 132, 117

Fan, X., et al. 2001, AJ, 121, 54

Fardal, M. A., Giroux, M. L., \& Shull, J. M. 1998, AJ, 115, 2206

Fardal, M. A., \& Shull, J. M. 1993, ApJ, 415, 524

Faucher-Giguère, C.-A., Lidz, A., Hernquist, L., \& Zaldarriaga, M. 2008a, ApJ, 682, L9

Faucher-Giguère, C.-A., Lidz, A., Hernquist, L., \& Zaldarriaga, M. 2008b, ApJ, 688,85

Faucher-Giguère, C.-A., Lidz, A., Zaldarriaga, M., \& Hernquist, L. 2008c, ApJ, 673,39

Faucher-Giguère, C.-A., Prochaska, J. X., Lidz, A., Hernquist, L., \& Zaldarriaga, M. 2008d, ApJ, 681, 831

Fechner, C., \& Richter, P. 2009, A\&A, 496, 31

Fontanot, F., Cristiani, S., Monaco, P., Nonino, M., Vanzella, E., Brandt, W. N., Grazian, A., \& Mao, J. 2007, A\&A, 461, 39

Furlanetto, S. 2008, arXiv:0812.3411

Furlanetto, S. 2009, ApJ, 700, 1666

Furlanetto, S. R., \& Oh, S. P. 2008, ApJ, 681, 1

Gilli, R., Comastri, A., \& Hasinger, G. 2007, A\&A, 463, 79

Giroux, M. L., \& Shapiro, P. R. 1996, ApJS, 102, 191 
Gleser, L., Nusser, A., Benson, A. J., Ohno, H., \& Sugiyama, N. 2005, MNRAS, 361, 1399

Gnedin, N. Y., \& Hamilton, A. J. S. 2002, MNRAS, 334, 107

Green, J. C. 2000, Proc. SPIE, 4013, 352

Gunn, J. E., \& Peterson, B. A. 1965, ApJ, 142, 1633

Haardt, F., \& Madau, P. 1996, ApJ, 461, 20

Haardt, F., \& Madau, P. 2001, in Proc. 21st Moriond Astrophys. Meeting, Clusters of Galaxies and the High Redshift Universe Observed in X-rays, ed. D. M. Neumann \& J. T. V. Tran (Gif-sur-Yvette: Ed. Frontières)

Haehnelt, M. G., Madau, P., Kudritzki, R., \& Haardt, F. 2001, ApJ, 549, L151

Hasinger, G., Miyaji, T., \& Schmidt, M. 2005, A\&A, 441, 417

Hernquist, L., Katz, N., Weinberg, D. H., \& Miralda-Escudé, J. 1996, ApJ, 457, L51

Hernquist, L., \& Springel, V. 2003, MNRAS, 341, 1253

Hopkins, P. F., Hernquist, L., Cox, T. J., Di Matteo, T., Martini, P., Robertson, B., \& Springel, V. 2005a, ApJ, 630, 705

Hopkins, P. F., Hernquist, L., Cox, T. J., Di Matteo, T., Robertson, B., \& Springel, V. 2006, ApJS, 163, 1

Hopkins, P. F., Hernquist, L., Cox, T. J., \& Kereš, D. 2008, ApJS, 175, 356

Hopkins, P. F., Hernquist, L., Martini, P., Cox, T. J., Robertson, B., Di Matteo, T., \& Springel, V. 2005b, ApJ, 625, L71

Hopkins, P. F., Richards, G. T., \& Hernquist, L. 2007, ApJ, 654, 731

Hui, L., \& Gnedin, N. Y. 1997, MNRAS, 292, 27

Hui, L., \& Haiman, Z. 2003, ApJ, 596, 9

Jena, T., et al. 2005, MNRAS, 361, 70

Kallman, T., \& McCray, R. 1980, ApJ, 242, 615

Katz, N., Weinberg, D. H., \& Hernquist, L. 1996a, ApJS, 105, 19

Katz, N., Weinberg, D. H., Hernquist, L., \& Miralda-Escude, J. 1996b, ApJ, 457, L57

Kewley, L. J., Dopita, M. A., Sutherland, R. S., Heisler, C. A., \& Trevena, J. 2001, ApJ, 556, 121

Kim, T.-S., Carswell, R. F., Cristiani, S., D’Odorico, S., \& Giallongo, E. 2002, MNRAS, 335, 555

Kim, T.-S., Cristiani, S., \& D’Odorico, S. 2001, A\&A, 373, 757

Kirkman, D., et al. 2005, MNRAS, 360, 1373

Komatsu, E., et al. 2009, ApJS, 180, 330

Loeb, A. 2009, J. Cosmol. Astropart. Phys., JCAP03(2009)022

Madau, P., \& Haardt, F. 2009, ApJ, 693, L100

Madau, P., Haardt, F., \& Rees, M. J. 1999, ApJ, 514, 648

Madau, P., \& Phinney, E. S. 1996, ApJ, 456, 124

Maselli, A., Ciardi, B., \& Kanekar, A. 2009, MNRAS, 393, 171

Maselli, A., \& Ferrara, A. 2005, MNRAS, 364, 1429

McDonald, P., \& Miralda-Escudé, J. 2001, ApJ, 549, L11

McDonald, P., Miralda-Escudé, J., Rauch, M., Sargent, W. L. W., Barlow, T. A., \& Cen, R. 2001, ApJ, 562, 52

McDonald, P., Miralda-Escudé, J., Rauch, M., Sargent, W. L. W., Barlow, T. A., Cen, R., \& Ostriker, J. P. 2000, ApJ, 543, 1

McQuinn, M., Lidz, A., Zaldarriaga, M., Hernquist, L., Hopkins, P. F., Dutta, S., \& Faucher-Giguere, C. 2009, ApJ, 694, 842

Meiksin, A., \& White, M. 2003, MNRAS, 342, 1205

Meiksin, A., \& White, M. 2004, MNRAS, 350, 1107

Miniati, F., Ferrara, A., White, S. D. M., \& Bianchi, S. 2004, MNRAS, 348, 964

Miralda-Escudé, J. 2003, ApJ, 597, 66

Miralda-Escude, J., \& Ostriker, J. P. 1990, ApJ, 350, 1

Misawa, T., Tytler, D., Iye, M., Kirkman, D., Suzuki, N., Lubin, D., \& Kashikawa, N. 2007, AJ, 134, 1634

Miyaji, T., Hasinger, G., \& Schmidt, M. 2000, A\&A, 353, 25

Neufeld, D. A. 1990, ApJ, 350, 216

Oh, S. P. 2001a, ApJ, 553, 25

Oh, S. P. 2001b, ApJ, 553, 499

O’Shea, B. W., Bryan, G., Bordner, J., Norman, M. L., Abel, T., Harkness, R., \& Kritsuk, A. 2004, arXiv:astro-ph/0403044

Osterbrock, D. E., \& Ferland, G. J. 2006, in Astrophysics of Gaseous Nebulae and Active Galactic Nuclei, ed. D. E. Osterbrock \& G. J. Ferland (2nd ed; Sausalito, CA: Univ. Science Books)

Ouchi, M., et al. 2008, ApJS, 176, 301

Paresce, F., McKee, C. F., \& Bowyer, S. 1980, ApJ, 240, 387

Paschos, P., Norman, M. L., Bordner, J. O., \& Harkness, R. 2007, arXiv:0711.1904

Pearce, F. R., \& Couchman, H. M. P. 1997, New Astron., 2, 411
Quinn, T., Katz, N., \& Efstathiou, G. 1996, MNRAS, 278, L49

Rauch, M., et al. 1997, ApJ, 489, 7

Razzaque, S., Dermer, C. D., \& Finke, J. D. 2009, ApJ, 697, 483

Reddy, N. A., Steidel, C. C., Pettini, M., Adelberger, K. L., Shapley, A. E., Erb, D. K., \& Dickinson, M. 2008, ApJS, 175, 48

Richards, G. T., et al. 2005, MNRAS, 360, 839

Richards, G. T., et al. 2006, AJ, 131, 2766

Ricotti, M., Gnedin, N. Y., \& Shull, J. M. 2000, ApJ, 534, 41

Ricotti, M., \& Ostriker, J. P. 2004, MNRAS, 352, 547

Rybicki, G. B., \& Lightman, A. P. 1979, Radiative Processes in Astrophysics (New York: Wiley Interscience), 393

Sawicki, M., \& Thompson, D. 2006, ApJ, 642, 653

Schaye, J. 2001, ApJ, 559, 507

Schaye, J., Aguirre, A., Kim, T.-S., Theuns, T., Rauch, M., \& Sargent, W. L. W. 2003, ApJ, 596, 768

Schaye, J., Theuns, T., Rauch, M., Efstathiou, G., \& Sargent, W. L. W. 2000, MNRAS, 318, 817

Scott, J., Bechtold, J., Morita, M., Dobrzycki, A., \& Kulkarni, V. P. 2002, ApJ, 571,665

Scott, J. E., Kriss, G. A., Brotherton, M., Green, R. F., Hutchings, J., Shull, J. M., \& Zheng, W. 2004, ApJ, 615, 135

Shapiro, P. R., Giroux, M. L., \& Babul, A. 1994, ApJ, 427, 25

Shapley, A. E., Steidel, C. C., Pettini, M., \& Adelberger, K. L. 2003, ApJ, 588, 65

Shapley, A. E., Steidel, C. C., Pettini, M., Adelberger, K. L., \& Erb, D. K. 2006, ApJ, 651, 688

Shull, J. M., Tumlinson, J., Giroux, M. L., Kriss, G. A., \& Reimers, D. 2004, ApJ, 600,570

Siana, B., et al. 2008, ApJ, 675, 49

Simcoe, R. A., Sargent, W. L. W., \& Rauch, M. 2004, ApJ, 606, 92

Sokasian, A., Abel, T., \& Hernquist, L. 2002, MNRAS, 332, 601

Songaila, A. 2001, ApJ, 561, L153

Songaila, A., \& Cowie, L. L. 1996, AJ, 112, 335

Springel, V. 2005, MNRAS, 364, 1105

Springel, V., \& Hernquist, L. 2003, MNRAS, 339, 312

Springel, V., Yoshida, N., \& White, S. D. M. 2001, New Astron., 6, 79

Steidel, C. C., Adelberger, K. L., Giavalisco, M., Dickinson, M., \& Pettini, M. 1999, ApJ, 519, 1

Steidel, C. C., Pettini, M., \& Adelberger, K. L. 2001, ApJ, 546, 665

Stengler-Larrea, et al. 1995, ApJ, 444, 64

Storrie-Lombardi, L. J., Irwin, M. J., \& McMahon, R. G. 1996, MNRAS, 282, 1330

Storrie-Lombardi, L. J., McMahon, R. G., Irwin, M. J., \& Hazard, C. 1994, ApJ, 427, L13

Syphers, D., et al. 2009, ApJ, 690, 1181

Telfer, R. C., Zheng, W., Kriss, G. A., \& Davidsen, A. F. 2002, ApJ, 565, 773

Theuns, T., Bernardi, M., Frieman, J., Hewett, P., Schaye, J., Sheth, R. K., \& Subbarao, M. 2002, ApJ, 574, L111

Theuns, T., Leonard, A., \& Efstathiou, G. 1998, MNRAS, 297, L49

Thoul, A. A., \& Weinberg, D. H. 1996, ApJ, 465, 608

Tittley, E. R., \& Meiksin, A. 2007, MNRAS, 380, 1369

Tytler, D., et al. 2004, ApJ, 617, 1

Ueda, Y., Akiyama, M., Ohta, K., \& Miyaji, T. 2003, ApJ, 598, 886

Verhamme, A., Schaerer, D., \& Maselli, A. 2006, A\&A, 460, 397

Weinberg, D. H., Hernquist, L., \& Katz, N. 1997, ApJ, 477, 8

Weymann, R. J., et al. 1998, ApJ, 506, 1

Weymann, R. J., \& Williams, R. E. 1969, ApJ, 157, 1201

Wolfe, A. M., Gawiser, E., \& Prochaska, J. X. 2005, ARA\&A, 43, 861

Wyithe, J. S. B., \& Loeb, A. 2003, ApJ, 586, 693

Yoshida, M., et al. 2006, ApJ, 653, 988

Zahn, O., Lidz, A., McQuinn, M., Dutta, S., Hernquist, L., Zaldarriaga, M., \& Furlanetto, S. R. 2007, ApJ, 654, 12

Zaldarriaga, M., Hui, L., \& Tegmark, M. 2001, ApJ, 557, 519

Zheng, W., et al. 2004, ApJ, 605, 631

Zheng, W., Kriss, G. A., Telfer, R. C., Grimes, J. P., \& Davidsen, A. F. 1997, ApJ, 475,469

Zheng, Z., \& Miralda-Escudé, J. 2002, ApJ, 578, 33

Zuo, L. 1992a, MNRAS, 258, 36

Zuo, L. 1992b, MNRAS, 258, 45

Zuo, L., \& Phinney, E. S. 1993, ApJ, 418, 28 